\title{
Optimal Control and Temperature Variations of Malaria Transmission Dynamics
}

\author{
Folashade B. Agusto iD \\ Department of Ecology and Evolutionary Biology, University of Kansas, Lawrence, KS, USA \\ Correspondence should be addressed to Folashade B. Agusto; fbagusto@gmail.com
}

Received 28 April 2020; Revised 5 October 2020; Accepted 21 October 2020; Published 28 November 2020

Academic Editor: Chongyang Liu

Copyright (c) 2020 Folashade B. Agusto. This is an open access article distributed under the Creative Commons Attribution License, which permits unrestricted use, distribution, and reproduction in any medium, provided the original work is properly cited.

\begin{abstract}
Malaria is a Plasmodium parasitic disease transmitted by infected female Anopheles mosquitoes. Climatic factors, such as temperature, humidity, rainfall, and wind, have significant effects on the incidence of most vector-borne diseases, including malaria. The mosquito behavior, life cycle, and overall fitness are affected by these climatic factors. This paper presents the results obtained from investigating the optimal control strategies for malaria in the presence of temperature variation using a temperature-dependent malaria model. The study further identified the temperature ranges in four different geographical regions of sub-Saharan Africa, suitable for mosquitoes. The optimal control strategies in the temperature suitable ranges suggest, on average, a high usage of both larvicides and adulticides followed by a moderate usage of personal protection such as bednet. The average optimal bednet usage mimics the solution profile of the mosquitoes as the mosquitoes respond to changes in temperature. Following the results from the optimal control, this study also investigates using a temperature-dependent model with insecticidesensitive and insecticide-resistant mosquitoes the impact of insecticide-resistant mosquitoes on disease burden when temperature varies. The results obtained indicate that optimal bednet usage on average is higher when insecticide-resistant mosquitoes are present. Besides, the average bednet usage increases as temperature increases to the optimal temperature suitable for mosquitoes, and it decreases after that, a pattern similar to earlier results involving insecticide-sensitive mosquitoes. Thus, personal protection, particularly the use of bednets, should be encouraged not only at low temperatures but particularly at high temperatures when individuals avoid the use of bednets. Furthermore, control and reduction of malaria may be possible even when mosquitoes develop resistance to insecticides.
\end{abstract}

\section{Introduction}

Malaria is a Plasmodium parasitic infectious disease transmitted by infected female anopheles mosquitoes. SubSaharan Africa and many tropical and subtropical regions of the world experience a large number of malaria-related mortality and morbidity with most of the disease burden in sub-Saharan Africa [1]. Climatic factors, such as temperature, humidity, rainfall, wind, duration of daylight, and vapor pressure, significantly affect the incidence of many vector-borne diseases, including malaria $[2,3]$. These climatic factors affect the host, vector, and parasite behavior. They also affect the duration of the vector and parasite/ pathogen life cycles [4-7].
The average global surface temperatures since the late $19^{\text {th }}$ century have increased by about $0.5^{\circ} \mathrm{C}-0.6^{\circ} \mathrm{C}[3,8]$; unfortunately, due to tropical warming, this trend is expected to continue [8]. Although there is significant spatial heterogeneity in the anticipated changes in the global average, the greater temperature increase is expected in subSaharan Africa [8].

Variability in weather and climate determines the geographical distribution, seasonality, yearly variability, and longer-term trends of malaria transmission $[9,10]$. The distribution and duration of the disease are modified by periods of unusually high rainfall, altered humidity, or warmer temperatures leading to increased transmission [11]. Similarly, periods of long-term drought can reduce malaria 
transmission [9-11]. Variability in the El Niño cycle and other long-term meteorological cycles can increase the disease burden and periodic upsurges in cases resulting in malaria epidemics $[9,11]$.

The greatest impact due to climate change is likely to be observed in temperature ranges $14^{\circ} \mathrm{C}-18^{\circ} \mathrm{C}$ and $35^{\circ} \mathrm{C}-40^{\circ} \mathrm{C}$. These temperature ranges have a significant impact on the transmission of many vector-borne diseases [12]. Places at the lower end of the temperature range $\left(14^{\circ} \mathrm{C}-18^{\circ} \mathrm{C}\right)$ will see an increase or extension in the transmission periods [13]. For malaria transmission, many temperature-related effects have been documented and established. For example, the female adult anopheles mosquitoes tend to feed more frequently as temperature increases because blood is digested more quickly. However, the juvenile mosquitoes maturate more quickly in warmer waters as a result of the shortened incubation period (leading to a faster growth rate of the vector larvae) $[12,14]$. The average lifespan of an adult female mosquito is about 21 days, and this is also affected by temperature; the mosquito survival rapidly decreases when temperature rises beyond $\left[30^{\circ} \mathrm{C}, 32^{\circ} \mathrm{C}\right]$ [15]. Furthermore, temperature equally affects the malaria parasites inside the vector; at $22^{\circ} \mathrm{C}$, it takes 19 days for these parasites to mature within the mosquito, but the maturation period decreases to eight days at $30^{\circ} \mathrm{C}[15,16]$.

Temperatures greater than $34^{\circ} \mathrm{C}$ (extreme temperature) negatively affect the survival of malaria vectors, parasites, and, invariably, the transmission rate [17]. A shift in the distribution of vectors into nonendemic areas is expected as global temperature increases so that an increase in malaria incidence is expected in malaria-endemic areas with lower temperatures, while a decline in incidence is expected in regions at temperatures beyond the transmission peak (provided all other factors remain constant and do not change) $[18,19]$.

Furthermore, malaria transmission is enhanced by rainfall, which generally increases the availability and productivity (conditional on ambient temperatures) of mosquito breeding sites $[20,21]$. On the contrary, excess rainfall can lead to the flushing out of Anopheles breeding sites [22] and a decrease in the mosquito population.

Agusto et al. in [2] assessed the role of temperature variations on malaria transmission and identified mean monthly temperature ranges where malaria-associated burden increases in West African cities as $\left[22.61^{\circ} \mathrm{C}, 28.58^{\circ} \mathrm{C}\right]$, in Central African cities as $\left[16.68^{\circ} \mathrm{C}, 27.92^{\circ} \mathrm{C}\right]$, in East African cities as $\left[19.04^{\circ} \mathrm{C}, 26.75^{\circ} \mathrm{C}\right]$, and in KwaZulu-Natal, South Africa, as $\left[16^{\circ} \mathrm{C}, 25^{\circ} \mathrm{C}\right]$. The study carried out uncertainty and sensitivity analysis using Latin hypercube sampling (LHS) and partial rank correlation coefficients (PRCC) to identify parameters with the most impact on the total number of infectious individuals as output function; the parameters with the most impact on the output are mosquito carrying capacity $\left(K_{V}\right)$, the mosquito biting rate $\left(b_{V}\right)$, the transmission probability per contact for susceptible mosquitoes $\left(\beta_{V}\right)$ and susceptible humans $\left(\beta_{H}\right)$, the maturity rate from larvae stage to adult mosquito $\left(\theta_{V}\right)$, and the human recruitment rate $\left(\Pi_{H}\right)$.
The study also carried out uncertainty and sensitivity analysis by incrementing temperature by $2^{\circ} \mathrm{C}$; they found that the larvae maturation rate $\left(\theta_{V}\right)$ has a minimum PRCC value at $T=24^{\circ} \mathrm{C}$ and a maximum value at $T=30^{\circ} \mathrm{C}$. Furthermore, the study showed that the mosquito biting rate $\left(b_{V}\right)$ has a maximum PRCC value at $T=26^{\circ} \mathrm{C}$ and a minimum PRCC value occurs at $T=24^{\circ} \mathrm{C}$. The implication of this result on control policy is that when the mean monthly temperatures are in the range $[16.7,25]^{\circ} \mathrm{C}$, individuals in the community should use both mosquito-reduction strategy and personal protection against mosquito bites; however, for higher mean monthly temperatures in the range $[26,34]^{\circ} \mathrm{C}$, mosquito-reduction strategy should be emphasized in the community (or household) ahead of personal protection.

This study aims to determine if this should be the control protocol. In particular, should individuals use bednets when temperatures are low? Or should they desist from using bednets when temperatures are high? To address these questions, we apply optimal control theory using the results from the sensitivity analysis carried out in [2] to identify the time-dependent control variables to be implemented in our study.

The paper is organized as follows: the model formulation is given in Section 2, alongside details of the temperaturedependent parametrization adopted. The optimal control problem is given in Section 3. The section further includes the optimal control analysis and the characterization of the control variables. In Section 4, we give results of the numerical simulation of the control problem. In Section 5, we introduce a mathematical model with insecticide-resistant mosquitoes; this is due to the results obtained from applying the optimal controls in Section 4.1 involving insecticidesensitive mosquitoes. The optimal control analysis and numerical simulation for insecticide-resistant mosquitoes are implemented in Section 5.1. Finally, the discussion and conclusions are stated in Sections 6 and 7, respectively.

\section{Methods}

2.1. Model Formulation. In this paper, we consider the simple malaria model used in the study of Agusto et al. [2] since the model with the property of gradual boosting of immunity against malaria due to repeated exposure has marginal (or no) effect on predictions of disease burden. The human population, of size $N_{H}(t)$, is split into mutually exclusive subpopulations of individuals who are susceptible $\left(S_{H}(t)\right)$, exposed $\left(E_{H}(t)\right)$, infectious $\left(I_{H}(t)\right)$, and recovered from the infection $\left(R_{H}(t)\right)$ so that

$$
N_{H}(t)=S_{H}(t)+E_{H}(t)+I_{H}(t)+R_{H}(t) .
$$

Similarly, the total mosquito population size at time $t$, denoted by $N_{V}(t)$, is subdivided into immature mosquitoes (eggs, larvae, and pupae) $\left(L_{V}(t)\right)$, susceptible mosquitoes $\left(S_{V}(t)\right)$, exposed mosquitoes $\left(E_{V}(t)\right)$, and infectious mosquitoes $\left(I_{V}(t)\right)$.

The simple model is given by the following deterministic, nonautonomous, system of nonlinear differential equations, 
where dot notation denotes the derivative with respect to time $t$ and $T$ and $\widehat{T}=+\delta_{T}$ denote air and water temperature:

$$
\begin{aligned}
& \dot{S_{H}}(t)=\Pi_{H}+\omega_{H} R_{H}(t)-\lambda_{H}\left(T, I_{V}, N_{H}\right) S_{H}(t)-\mu_{H} S_{H}(t), \\
& \dot{E_{H}}(t)=\lambda_{H}\left(T, I_{V}, N_{H}\right) S_{H}(t)-\left(\sigma_{H}+\mu_{H}\right) E_{H}(t), \\
& \dot{I_{H}}(t)=\sigma_{H} E_{H}(t)-\left(\gamma_{H}+\mu_{H}+\delta_{H}\right) I_{H}(t), \\
& \dot{R_{H}}(t)=\gamma_{H} I_{H}(t)-\left(\omega_{H}+\mu_{H}\right) R_{H}(t), \\
& \dot{L_{V}}(t)=\phi_{V}(T)\left[1-\frac{L_{V}(t)}{K_{V}}\right]\left[S_{V}(t)+E_{V}(t)+I_{V}(t)\right]-\left[\theta_{V}(\widehat{T})+\mu_{L}(\widehat{T})\right] L_{V}(t), \\
& \dot{S_{V}}(t)=\theta_{V}(\widehat{T}) L_{V}(t)-\lambda_{V}\left(T, N_{H}\right) S_{V}(t)-\mu_{V}(T) S_{V}(t), \\
& \dot{E_{V}}(t)=\lambda_{V}\left(T, N_{H}\right) S_{V}(t)-\left[\sigma_{V}(T)+\mu_{V}(T)\right] E_{V}(t), \\
& \dot{I_{V}}(t)=\sigma_{V}(T) E_{V}(t)-\mu_{V}(T) I_{V}(t) .
\end{aligned}
$$

The flow diagram of malaria model (2) is shown in Figure 1; Table 1 describes the state variables and parameters.

The parameter $\Pi_{H}$ in the system (2) is the human recruitment rate, $\lambda_{H}\left(T, I_{V}(t), N_{H}(t)\right)$ is the temperaturedependent force of infection of healthy susceptible humans (after contact with infectious mosquitoes), and $\mu_{H}$ is the natural death rate of humans. The infection rate, $\lambda_{H}\left(T, I_{V}(t), N_{H}(t)\right)$, is defined as

$$
\lambda_{H}\left(T, I_{V}(t), N_{H}(t)\right)=\frac{\beta_{H} b_{V}(T) I_{V}(t)}{N_{H}(t)},
$$

where $\beta_{H}$ is the susceptible human transmission probability of malaria infection per bite from an infectious mosquito and $b_{V}(T)$ is the mosquito temperature-dependent per capita biting rate; the conservation law of mosquito bites was used to derive the temperature-dependent force of infection $\lambda_{H}\left(T, I_{V}(t), N_{H}(t)\right)$ [23-25]. The parameter, $\sigma_{H}$, represents exposed humans progression rate into the infectious class $\left(I_{H}\right)$. Also, the parameter $\gamma_{H}$ represents the rate at which individual in the $I_{H}$ class recovers (and moves to the recovered $R_{H}$ class). The parameter $\omega_{H}$ represents the rate at which the recovered individuals in $R_{H}$ loose their infectionacquired immunity and move to the $S_{H}$ class. The parameter $\delta_{H}$ is the rate at which individuals in the infectious human class $\left(I_{H}\right)$ suffer malaria-induced mortality.

After successfully obtaining blood meals from human hosts, the female mosquitoes will rest for a few days allowing the blood to digest and their eggs to develop. The process of mosquito egg laying is sensitive and dependent on temperature [26]; the laid eggs typically hatches within 2-3 days and may take up to 2-3 weeks in colder temperate climates $[26,27]$. The temperature-dependent egg deposition rate is denoted by $\phi_{V}(T)$. We assume that the immature mosquito population (comprising of the larvae and pupae) is limited by the carrying capacity $K_{V}$; this parameter is related to the amount of available nutrients and space [28-30]. Thus, $\phi_{V}(T)\left[1-L_{V}(t) / K_{V}\right]\left[S_{V}(t)+E_{V}(t)+I_{V}(t)\right]$ represents the logistic growth rate for the immature mosquitoes. The parameter $\theta_{V}(\widehat{T})$ denotes the temperature-dependent metamorphosis rate (i.e., maturation rate) of immature mosquitoes [31-34], and the temperature-dependent mortality rate of immature mosquitoes is give by the parameter $\mu_{L}(T)$ (it is worth noting that, in this study, the dynamics of the three aquatic stages of the mosquito is modeled using the single compartment, $L_{V}(t)$, for mathematical convenience (see also $[25,29,30])$ ). Susceptible adult female mosquitoes become infected, following effective contact with an infectious human (from an infectious blood meal); this force of infection is also temperature-dependent, and it is given by

$$
\lambda_{V}\left(T, I_{H}(t), N_{H}(t)\right)=\frac{\beta_{V} b_{V}(T) I_{H}(t)}{N_{H}(t)},
$$

where $\beta_{V}$ is the probability that a bite from a susceptible mosquito to a human with infectious gametocytes leads to the infection of the mosquito. The parameter $\sigma_{V}(T)$ is the temperature-dependent progression rate of exposed mosquitoes $[35,36]$. The parameter $\mu_{V}(T)$ represents the temperature-dependent death rate of adult mosquitoes; this parameter is dependent both on temperature and humidity and the ability of the mosquito to successfully obtain blood meals while avoiding host defenses [26]. We assume there is no disease-related death for the mosquitoes.

Following Agusto et al. [2], the temperature-dependent parameters of the model (2) are defined as follows: using the 

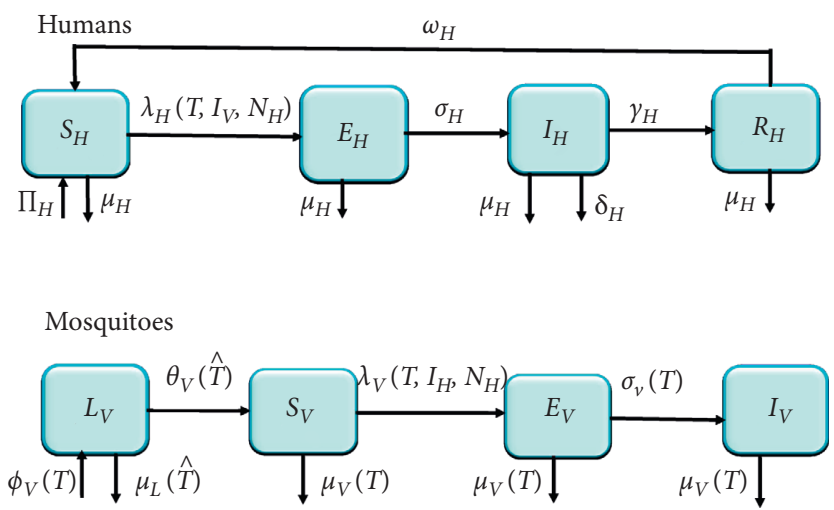

Figure 1: The malaria model (2) flow diagram.

quadratic functions used in [37], the mosquito biting rate, $b_{V}(T)$, and egg deposition rate, $\phi_{V}(T)$, are given by

$$
\begin{aligned}
& b_{V}(T)=-0.00014 T^{2}+0.027 T-0.322, \\
& \phi_{V}(T)=-0.153 T^{2}+8.61 T-97.7 .
\end{aligned}
$$

Similarly, the temperature-dependent parameters $\sigma_{V}(T), \theta_{V}(T)$, and $\mu_{V}(T)$ are defined in [37] as follows:

$$
\begin{aligned}
\sigma_{V}(T) & =-0.00083 T^{2}+0.044 T-0.487, \\
\theta_{V}(\widehat{T}) & =\frac{B(T) p_{\mathrm{EA}}(\widehat{T})}{\tau_{E A}(\widehat{T})}, \\
\mu_{V}(T) & =-\ln \left(-0.000828 T^{2}+0.0367 T+0.522\right) .
\end{aligned}
$$

The temperature-dependent function $B(T)$ represents the lifetime number of eggs laid by the female mosquito, the probability that the egg laid survives to become an adult mosquito is represented by $p_{\mathrm{EA}}(\widehat{T})$, and the development time from egg to adult mosquito is represented by $\tau_{\mathrm{EA}}(\widehat{T})$
[37]. The temperature-dependent function, $B(T)$, is defined as

$$
B(T)=\frac{\operatorname{EFD}(T)}{\mu_{V}(T)},
$$

where $\operatorname{EFD}(T)$ is the number of eggs laid per female per day. Hence, $B(T)$, the total number of eggs laid by a mosquito (in a lifetime), is equal to the number of eggs laid per day multiplied by the average adult lifespan $\left(1 / \mu_{V}(T)\right)$ [37]. These temperature-dependent parameters are expressed as [37]

$$
\begin{aligned}
& \operatorname{EFD}(T)=-0.153 T^{2}+8.61 T-97.7, \\
& p_{E A}(\widehat{T})=-0.00924 \widehat{T}^{2}+0.453 \widehat{T}-4.77, \\
& \tau_{E A}(\widehat{T})=\frac{1}{\left(-0.00094 \widehat{T}^{2}+0.049 \widehat{T}-0.552\right)} .
\end{aligned}
$$

Hence, substituting the temperature-dependent function (9)-(12) into (7), we have

$$
\begin{aligned}
\theta_{V}(\widehat{T})= & \left(-0.153 T^{2}+8.61 T-97.7\right)\left(-0.00924 \widehat{T}^{2}+0.453 \widehat{T}-4.77\right) \\
& \times\left[-0.00094 \widehat{T}^{2}+0.049 \widehat{T}-0.552\right]\left[\frac{1}{-\ln \left(-0.000828 T^{2}+0.0367 T+0.522\right)}\right]
\end{aligned}
$$

Lastly, the parameter $\mu_{L}(\widehat{T})[38]$ is defined as

$$
\mu_{L}(\widehat{T})=\frac{1}{8.560+20.654\left[1+(\widehat{T} / 19.759)^{6.827}\right]^{-1}} .
$$

Numerical simulations of the model are carried out using the mean monthly temperatures from 2011 to 2013 for ten cities in sub-Saharan Africa (three cities each in West, Central, and East Africa and KwaZulu-Natal, South Africa) as were implemented in [2]. The cities in West Africa are NZerekore, Guinea; Bamako, Mali; and Niamey, Niger. The cities in Central Africa are Lubumbashi, DRC; Tchibanga,
Gabon; and Ati Chad, while the cities in East Africa are Kigali, Rwanda; Gulu, Uganda; and Lodwar, Kenya.

The temperature within the three cities of West, Central, and East Africa is chosen to represent the minimum, mean, and maximum mean monthly temperature, respectively, in the given region. As in [2], we used the temperature profile of KwaZulu-Natal as a representative sample of cities in southern Africa, since most cities in southern Africa have similar mean monthly temperatures [39].

The next section investigates the control strategies for curtailing malaria following the results obtained from sensitivity analysis in [2]. 
TABLE 1: Description of the variables and parameters of the malaria model (2).

\begin{tabular}{lc}
\hline Variable & Description \\
\hline$S_{H}(t)$ & Number of susceptible humans \\
$E_{H}(t)$ & Number of exposed humans \\
$I_{H}(t)$ & Number of infectious humans \\
$R_{H}(t)$ & Number of recovered humans \\
$L_{V}(t)$ & Number of immature (eggs, larva, and pupa stages) \\
$S_{V}(t)$ & vectors \\
$E_{V}(t)$ & Number of susceptible vectors \\
$I_{V}(t)$ & Number of exposed vectors \\
\hline Parameter & Number of infectious vectors \\
$\Pi_{H}$ & Description \\
$\beta_{H}$ & Transmission probability for susceptible humans per \\
$\beta_{V}$ & contact \\
$\sigma_{H}$ & Transmission probability for susceptible vectors per \\
$\mu_{H}$ & contact \\
$\delta_{H}$ & Progression rate of infectious humans \\
$\gamma_{H}$ & Humans natural mortality rate \\
$\omega_{H}$ & Humans disease-induced mortality rate \\
$K_{V}$ & Recovery rate \\
\hline & Larrying of immunity rate
\end{tabular}

time-dependent controls, namely, personal protection against mosquito bite and mosquito-reduction strategies. The mosquito-reduction strategy is further divided into two (larvicides and adulticides) according to the life stages of the mosquitoes. For simplicity, we assume that the use of bednets reduces the contact between mosquitoes and humans and that the mosquito population is reduced by the mosquito-reduction strategies (larvicides and adulticides), which we assume in this case chemical-based insecticides. However, there are other environmental friendly larvaciding methods that can be used to target the larvae stage of the mosquitoes, such as larvivorous fish which prey on the larvae [40-44] and bacterial larvicide which causes the mosquitoes to starve to death once they ingest the spores of the bacterial larvicide [45-48]. In a future study, we will incorporate the insecticide capacity of the bednets [49] and the actions and kinetics of these larvacide methods. Thus, the three timedependent control variables are $u_{H}(t), u_{L}(t)$, and $u_{V}(t)$, representing personal protection, larvaciding, and adulticiding. Thus, model (2) becomes:

\section{Optimal Control Problems}

Following the results obtained from the sensitivity analysis in [2], we introduce into the transmission model (2) two

$$
\begin{aligned}
\frac{\mathrm{d} S_{H}(t)}{\mathrm{d} t}= & \Pi_{H}+\omega_{H} R_{H}(t)-\lambda_{H}\left(T,\left[1-u_{H}(t) I_{V}, N_{H}\right]\right) S_{H}(t)-\mu_{H} S_{H}(t), \\
\frac{\mathrm{d} E_{H}(t)}{\mathrm{d} t}= & \lambda_{H}\left[T,\left(1-u_{H}(t)\right) I_{V}, N_{H}\right] S_{H}(t)-\left(\sigma_{H}+\mu_{H}\right) E_{H}(t), \\
\frac{\mathrm{d} I_{H}(t)}{\mathrm{d} t}= & \sigma_{H} E_{H}(t)-\left(\gamma_{H}+\mu_{H}+\delta_{H}\right) I_{H}(t), \\
\frac{\mathrm{d} R_{H}(t)}{\mathrm{d} t}= & \gamma_{H} I_{H}(t)-\left(\omega_{H}+\mu_{H}\right) R_{H}(t), \\
\frac{\mathrm{d} L_{V}(t)}{\mathrm{d} t}= & \phi_{V}(T)\left[1-\frac{L_{V}(t)}{K_{V}}\right]\left[S_{V}(t)+E_{V}(t)+I_{V}(t)\right] \\
& -\left[\theta_{V}(\widehat{T})+\mu_{L}(\widehat{T})\right] L_{V}(t)-u_{L}(t) L_{V}(t), \\
\frac{\mathrm{d} S_{V}(t)}{\mathrm{d} t}= & \theta_{V}(\widehat{T}) L_{V}(t)-\lambda_{V}\left[T,\left(1-u_{H}(t)\right) N_{H}\right] S_{V}(t)-\mu_{V}(T) S_{V}(t)-u_{V}(t) S_{V}(t), \\
\frac{\mathrm{d} E_{V}(t)}{\mathrm{d} t}= & \lambda_{V}\left[T,\left(1-u_{H}(t)\right) N_{H}\right] S_{V}(t)-\left[\sigma_{V}(T)+\mu_{V}(T)\right] E_{V}(t)-u_{V}(t) E_{V}(t), \\
\frac{\mathrm{d} I_{V}(t)}{\mathrm{d} t}= & \sigma_{V}(T) E_{V}(t)-\mu_{V}(T) I_{V}(t)-u_{V}(t) I_{V}(t),
\end{aligned}
$$

where 


$$
\begin{aligned}
\lambda_{H}\left[T,\left(1-u_{H}(t)\right) I_{V}(t), N_{H}(t)\right] & =\frac{\beta_{H}\left(1-u_{H}(t)\right) b_{V}(T) I_{V}(t)}{N_{H}(t)}, \\
\lambda_{V}\left[T,\left(1-u_{H}(t)\right) N_{H}\right] & =\frac{\beta_{V}\left(1-u_{H}(t)\right) b_{V}(T) I_{H}(t)}{N_{H}(t)} .
\end{aligned}
$$

The factor $\left(1-u_{H}(t)\right)$ reduces the force of infection associated with humans and mosquitoes, respectively [50-52].

This study aims to minimize the objective function defined as

$$
\begin{aligned}
J(u)= & \int_{0}^{t_{f}}\left[A_{1} I_{H}+B_{1} L_{V}+B_{2} S_{V}+B_{3} E_{V}+B_{4} I_{V}+C_{1} u_{H}\right. \\
& \left.+\varepsilon C_{2} u_{H}^{2}+C_{3} u_{L}+\varepsilon C_{4} u_{L}^{2}+C_{5} u_{V}+\varepsilon C_{6} u_{V}^{2}\right] \mathrm{d} t,
\end{aligned}
$$

subject to the differential equations (15), where $t_{f}$ is the final time. This performance specification involves minimizing the number of infected humans and the mosquito population, along with the cost of applying the controls $\left(u_{H}(t), u_{L}(t), u_{V}(t)\right)$. Often the cost of implementing a control would be nonlinear, and as such, a simple nonlinear quadratic objective functional is taken here. The costs can include funds needed for control implementation, cost of hospitalization, and the cost of manpower lost due to hospitalization. This assumption is based on the fact that there are no linear relationships between the effects of the intervention and the cost of the intervention on the infected populations; such quadratic costs have been frequently used [50-54]. The coefficients and balancing cost factors are $A_{1} B_{j}, j=1, \ldots, 4, C_{k}, k=1, \ldots, 6$. that

We seek to find the optimal controls $u_{H}^{*}, u_{L}^{*}$, and $u_{V}^{*}$ such

$$
J\left(u_{H}^{*}, u_{L}^{*}, u_{V}^{*}\right)=\min _{\mathcal{U}}\left\{J\left(u_{H}, u_{L}, u_{V}\right)\right\}
$$

The optimal control solution is given as

$$
\begin{aligned}
u_{H}^{*}= & \frac{1}{2 \varepsilon C_{2}}\left(-C_{1} N_{H}^{*}+S_{V}^{*} b_{V}(T) \beta_{V}\left(\lambda_{E_{V}}^{*}-\lambda_{S_{V}}^{*}\right) I_{H}^{*}\right. \\
& \left.+I_{V}^{*} b_{V}(T) \beta_{H}\left[\left(\lambda_{E_{H}}^{*}-\lambda_{S_{H}}^{*}\right) S_{H}^{*}-\lambda_{R_{H}}^{*} R_{H}^{*}\right]\right), \\
u_{L}^{*}= & \frac{\lambda_{L_{V}}^{*} L_{V}^{*}-C_{3}}{2 \varepsilon C_{4}}, \\
u_{V}^{*}= & \frac{\lambda_{E_{V}}^{*} E_{V}+\lambda_{I_{V}}^{*} I_{V}^{*}+\lambda_{S_{V}}^{*} S_{V}^{*}-C_{5}}{2 \varepsilon C_{6}} .
\end{aligned}
$$

The control characterization is given in Appendix A.

In the next section, the results of the numerical solutions of the optimality system, the corresponding optimal control, and the interpretations of the various cases are considered.

\section{Numerical Illustration}

The forward-backward sweep algorithm, along with the fourth-order Runge-Kutta method, was used to obtain the numerical solutions of the optimal controls and state values. The algorithm requires that initial estimates for the control variables be made. Then using these estimates, the state variables are then solved forward in time using the dynamics (15). The results obtained for the state variables are used in computing the solution for the adjoint equations (A.4) together with given final conditions (A.7). These are solved backward in time, using the backward fourthorder Runge-Kutta method. Both the solution of the state and adjoint values are then used to update the control, and the process is repeated until there is sufficient convergence between the current state, adjoint, and control values [55]. The algorithm is implemented using MATLAB R2016A [56].

Thus, to implement the optimal control algorithm, the following initial condition values are used $S_{H}(0)=9384291$, $E_{H}(0)=8200, \quad I_{H}(0)=1200, \quad R_{H}(0)=1000, \quad L_{V}(0)=$ $11277924, S_{V}(0)=44300, E_{V}(0)=1452$, and $I_{V}(0)=2800$. The weight factors are chosen as $A_{1}=B_{i}=C_{j}=1.00$, $i=1, \ldots, 4, j=1, \ldots, 6$. It should be pointed out that the values chosen for the initial conditions and weights used in the optimal control simulations are only of theoretical sense to demonstrate the control strategies proposed in this paper.

First, we consider the optimal control simulation for the maximum mean temperature of $25.32^{\circ} \mathrm{C}$ in KwaZulu-Natal, South Africa. This is the highest mean monthly temperature in the Province, and it occurs in November. The simulation is done for a period of 30 days representing the number of days in the month. In the next section, we consider the control using the mean monthly temperature over the twelve months in a year. The results of the optimal control simulations of model (15) are depicted in Figure 2. With optimal control, there are fewer infected humans in the community compared to the case with no control (Figure 2(a)). On the thirtieth day, there were a total of $3.3330 \times 10^{4}$ infected humans in the absence of control compared to approximately $8.7797 \times 10^{3}$ with the application of control. Similarly, for the infected mosquitoes, there are more infected mosquitoes in the absence of control, unlike when control is applied where the mosquitoes are significantly reduced (Figure (2(b)). In particular, there were $2.4505 \times 10^{4}$ infected mosquitoes in the absence of control on the thirtieth day compared to approximately 120 with the application of control.

The corresponding time-dependent controls $\left(u_{L}(t), u_{V}(t)\right.$, and $\left.u_{H}(t)\right)$ are depicted in Figure 3 . The timedependent controls $u_{L}(t)$ start at the upper bound of unity for the most part of the simulation period before reducing to the lower bound. Control $u_{V}(t)$ starts to be low at 0.165 but quickly rises to the upper bound for most of the simulation period. The control $u_{H}(t)$ starts at the upper bound for about eight days before gradually reducing to the lower bound.

In the next section, we consider the control using the mean monthly temperature over the twelve months in a year and determine the temperature range suitable for mosquitoes. The total number of infected humans and mosquitoes per mean monthly temperature is used as metric for mean optimal controls $u_{L}(t), u_{V}(t)$, and $u_{H}(t)$. We take only a single value for the total number of infected humans and 


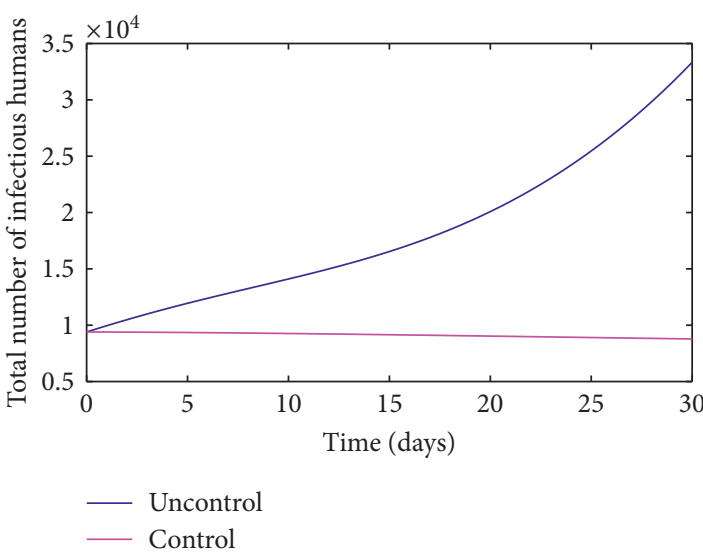

(a)

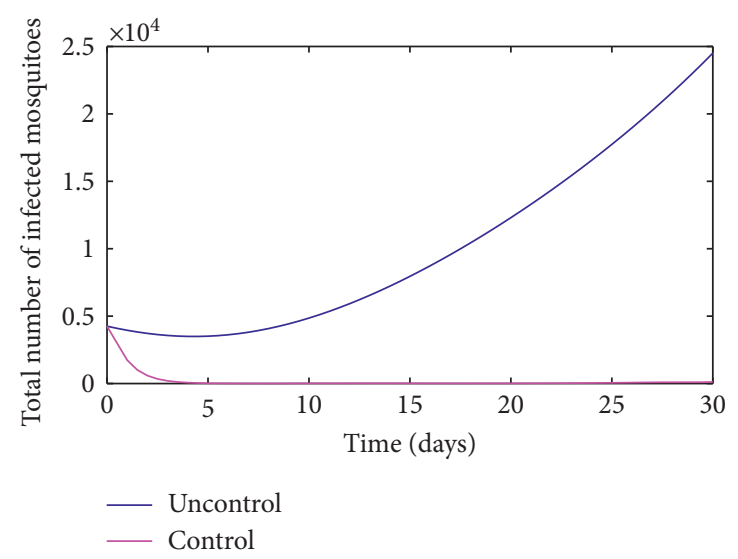

(b)

Figure 2: Simulation results of model (15) with and without controls using the maximum mean temperature of $25.32^{\circ} \mathrm{C}$ in KwaZulu-Natal, South Africa. (a) Infectious individuals. (b) Infectious mosquitoes.

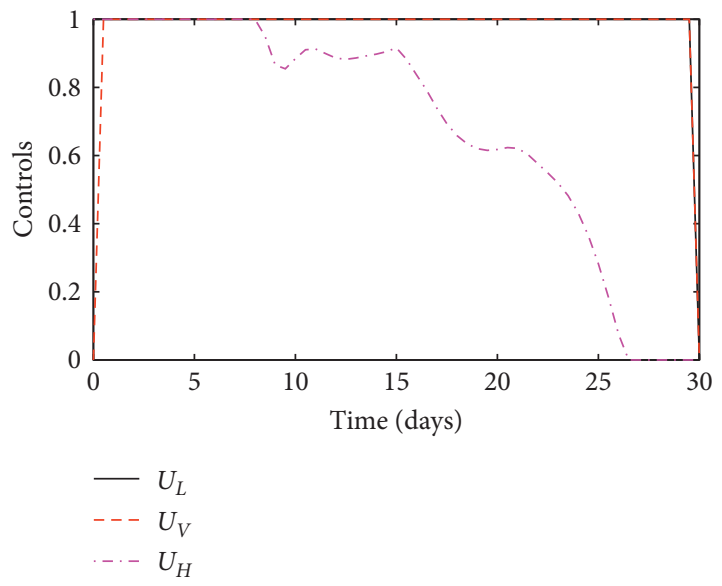

FIGURE 3: Simulation results of the control profile of model (15) using the maximum mean temperature of $25.32^{\circ} \mathrm{C}$ in $\mathrm{KwaZulu}-$ Natal, South Africa.

mosquitoes and the mean optimal control level for each control $u_{L}(t), u_{V}(t)$, and $u_{H}(t)$ since plotting the complete solution profiles will involve 36 solution trajectories resulting in a lot of figures to read and interpret. Therefore, using the total infected and the mean level of control, we can see clearly from the figures and draw interpretation quickly from the figures.

\subsection{Optimal Control with Mean Monthly Temperatures}

4.1.1. Mean Monthly Temperature: South Africa Profiles. The results of the optimal control simulations of the malaria model (2) in KwaZulu-Natal are depicted in Figures 4(a) and 4(b). First, the uncontrol and control cases for the mean monthly air temperatures in KwaZulu-Natal are superimposed with the total number of cases in each month generated using the model (2) with the baseline parameter values in Table 2. As previously obtained in [2], this figure show that, in general, malaria burden increases with increasing mean air temperature in the range $\left[16.7^{\circ} \mathrm{C}, 25.3^{\circ} \mathrm{C}\right]$. Figure 4(a) shows that there are fewer infected individuals with the application of the control measures.

Furthermore, the results of the optimal control simulations of the malaria model (2) measured in terms of the average control efforts are depicted in Figure 4(c). This shows that the time-dependent controls $u_{L}(t)$ and $u_{V}(t)$ on average are close to the upper bound throughout the year for all temperature values, while the time-dependent control $u_{H}(t)$ starts relatively low for low temperature and is relatively high for high temperature. It should be noted that the solution profile for $u_{H}(t)$ mimics the trajectory of the infected mosquitoes as the mosquitoes respond to variations in temperature (Figures $4(\mathrm{~b})$ and $4(\mathrm{c})$ ).

4.1.2. Mean Monthly Temperature: Other Cities Infected Human Profiles. Similar behavior is observed in the nine cities, used in [2], and three cities each were chosen from West, Central, and East African regions (Table 3). In particular, Figure 5 depicts the mean monthly air temperatures in the three cities in West Africa superimposed on the mean total number of new cases generated each month using model (2). The figures for Central and East African cities are depicted in Figures 6 and 7.

Figures 5(a)-5(e) show that in West Africa, malaria burden increases for temperatures in the range $\left[22.61^{\circ} \mathrm{C}, 28.58^{\circ} \mathrm{C}\right]$, while in Central Africa (see the infected humans in Figure 6), malaria burden increases for temperatures in the range $\left[16.68^{\circ} \mathrm{C}, 27.92^{\circ} \mathrm{C}\right]$. Lastly, the range in East Africa is $\left[19.04^{\circ} \mathrm{C}, 26.75^{\circ} \mathrm{C}\right.$ ] (Figure 7 for the infected humans). These simulations clearly show the variability in the suitable temperature ranges for malaria transmission in the various regions of sub-Saharan Africa, as previously obtained in [2]. The solution profile for infected mosquitoes is given Figures 8-10. It also shows that the solution profile 


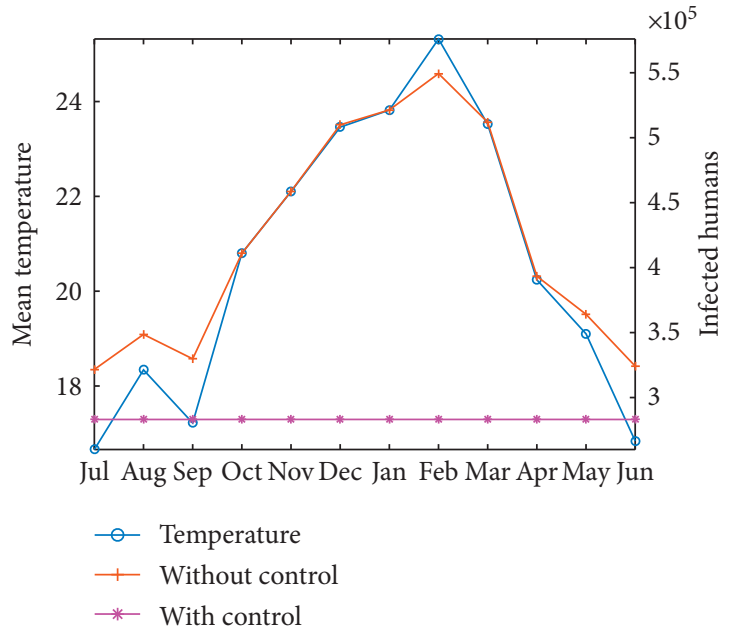

(a)

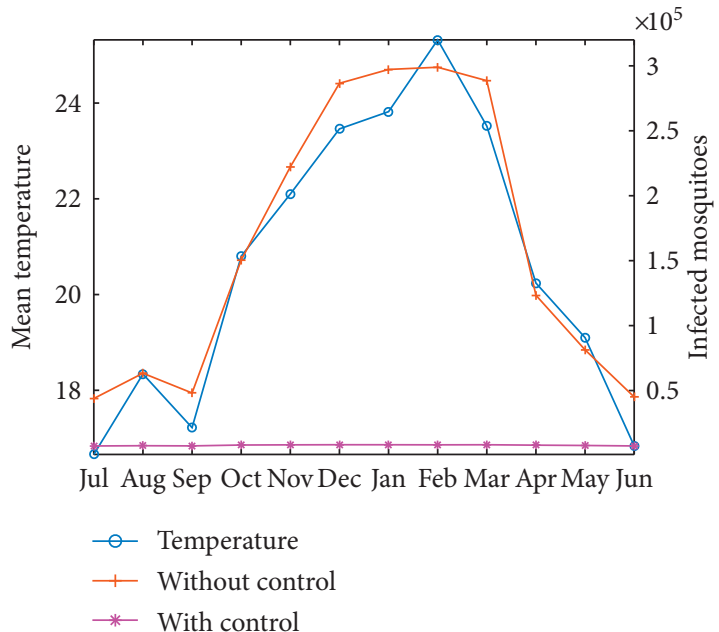

(b)

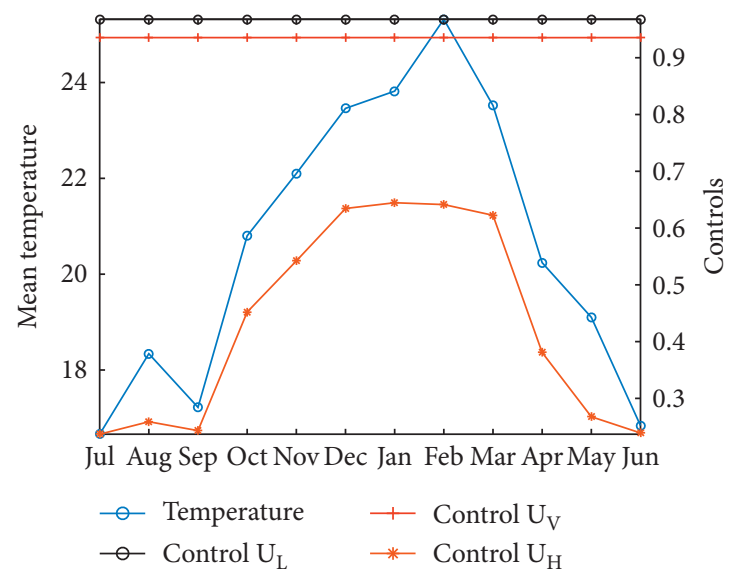

(c)

Figure 4: Simulations of the model (2) as a function of time plotting the monthly total number of infected humans and mosquitoes in KwaZulu-Natal, South Africa. (a) Total number of infected humans. (b) Total number of infected mosquitoes. (c) Optimal controls $u_{H}(t), u_{L}(t)$, and $u_{V}(t)$. The mean monthly temperature is plotted in blue lines and is superimposed onto each figure.

TABLe 2: Values and ranges of the temperature-independent parameters of the model (2).

\begin{tabular}{lccc}
\hline Parameter & Baseline value & Range & Reference \\
\hline$\Pi_{H}$ & $400 /$ day & $10-800 /$ day & Variable \\
$\beta_{H}$ & $0.24 /$ day & $0.072-0.64 /$ day & {$[25,57]$} \\
$\mu_{H}$ & $0.00004 /$ day & $(1 /((80 \times 365))-(1 /(70 \times 365))) /$ day & {$[25,57]$} \\
$\delta_{H}$ & $0.0003454 /$ day & $1.0 \times 10^{-15}-4.1 \times 10^{-4} /$ day & {$[25,57]$} \\
$\sigma_{H}$ & $(12 / 365) \times 3.04 /$ day & $0.067-0.20 /$ day & {$[25,57,58]$} \\
$\gamma_{H}$ & $0.0023 \pm 0.0005 /$ day & $0.0014-0.017 /$ day & {$[25,57]$} \\
$\omega_{H}$ & $1.7 \times 10^{-5} /$ day & $5.5 \times 10^{-5}-1.1 \times 10^{-2} /$ day & {$[25,57]$} \\
$K_{V}$ & 40000 & $5.0 \times 10^{1}-3.3 \times 10^{6}$ & {$[29,30]$} \\
\hline
\end{tabular}

TABLE 3: Cities in West, Central, and East African regions.

\begin{tabular}{lcc}
\hline West African cities & Central African cities & East African cities \\
\hline NZerekore, Guinea & Lubumbashi, DRC & Kigali, Rwanda \\
Bamako, Mali & Tchibanga, Gabon & Gulu, Uganda \\
Niamey, Niger & Ati, Chad & Lodwar, Kenya \\
\hline
\end{tabular}

for control $u_{H}(t)$ mimics the trajectory of the infected mosquitoes as the mosquitoes respond to variations in temperature (Figures 8-10).

Specifically, in West Africa, Figures 5(a), 5(c), and 5(e) show that there are fewer infected individuals with the application of the control measures. The average time- 


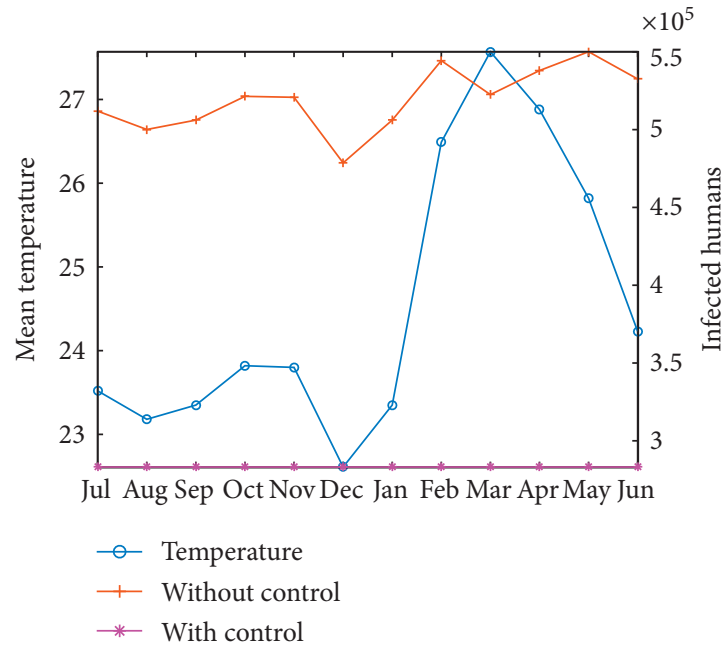

(a)

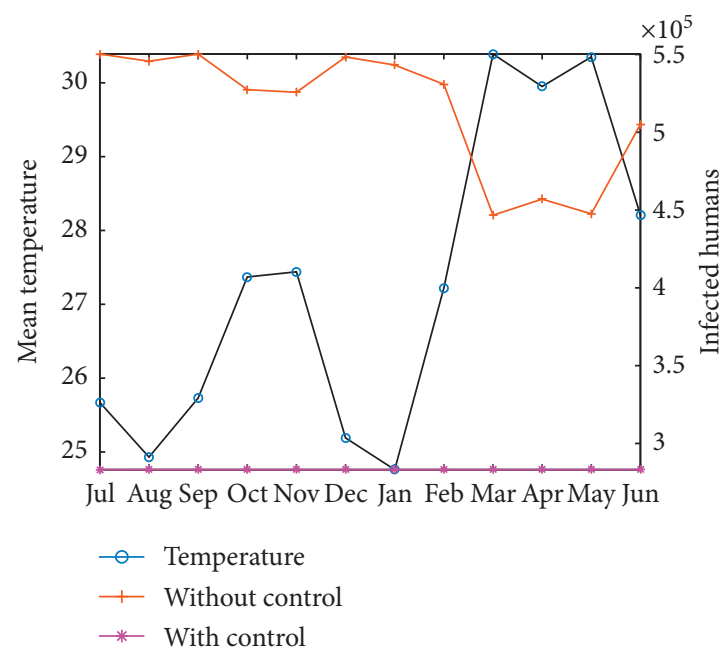

(c)

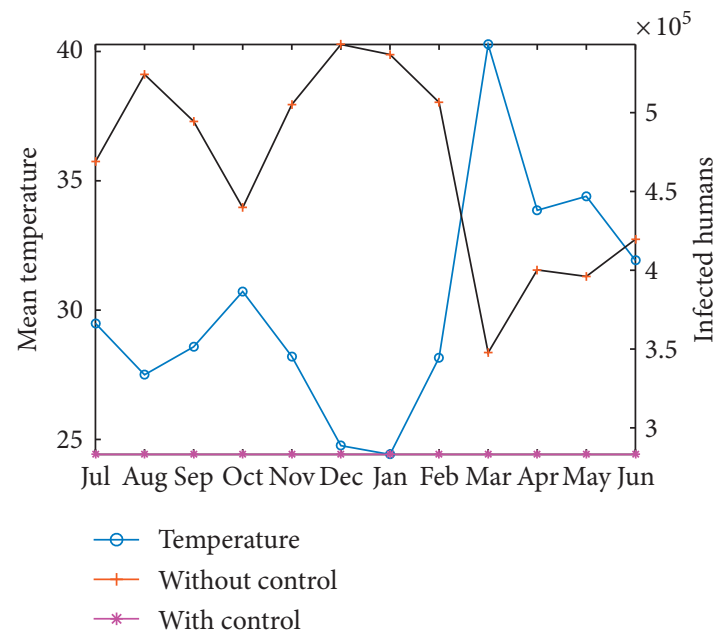

(e)

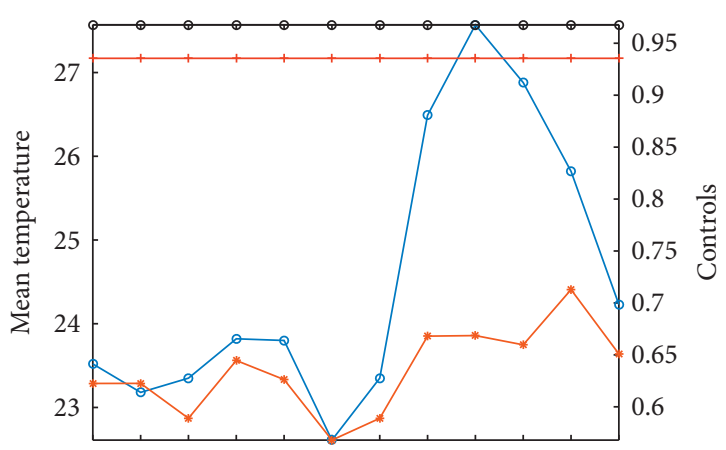

Jul Aug Sep Oct NovDec Jan Feb Mar Apr May Jun

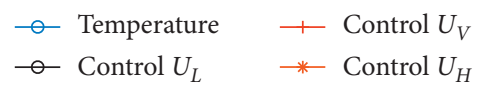

(b)

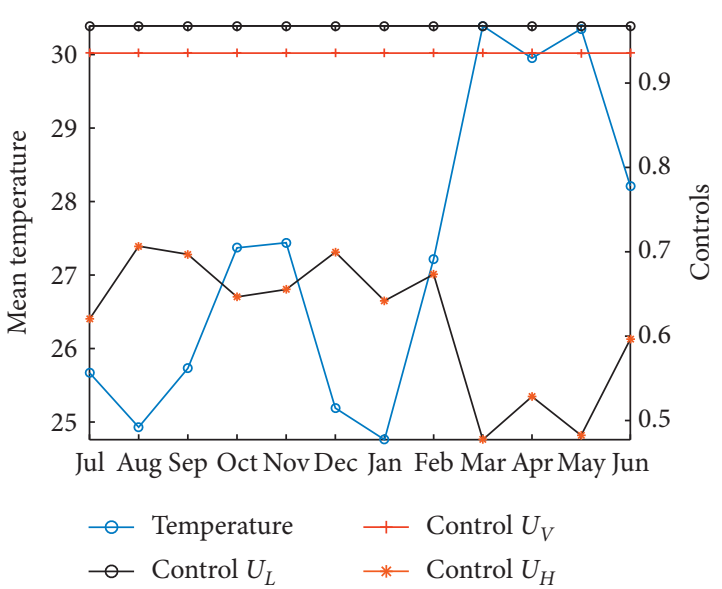

(d)

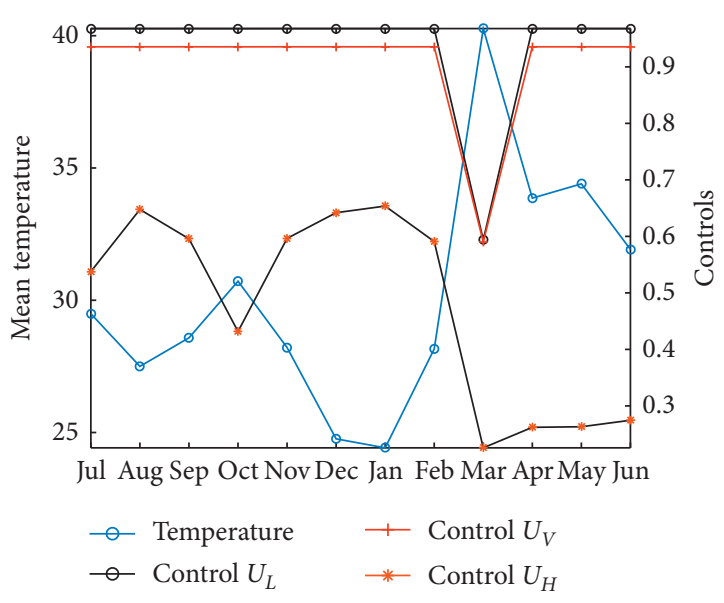

(f)

FIgURE 5: Simulations of the model (2) as a function of time: (a, c, e) the monthly total number of infected humans without and with control and $(\mathrm{b}, \mathrm{d}, \mathrm{f})$ optimal controls $u_{H}(t), u_{L}(t)$, and $u_{V}(t)$, in NZerekore, Guinea; Bamako, Mali; and Niamey, Niger; in West Africa. The mean monthly temperature is plotted in blue lines and is superimposed onto each figure. 


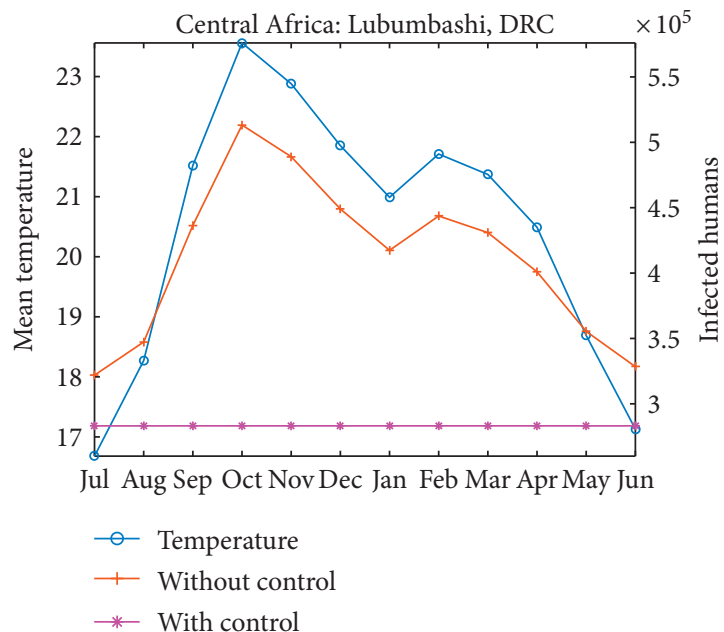

(a)

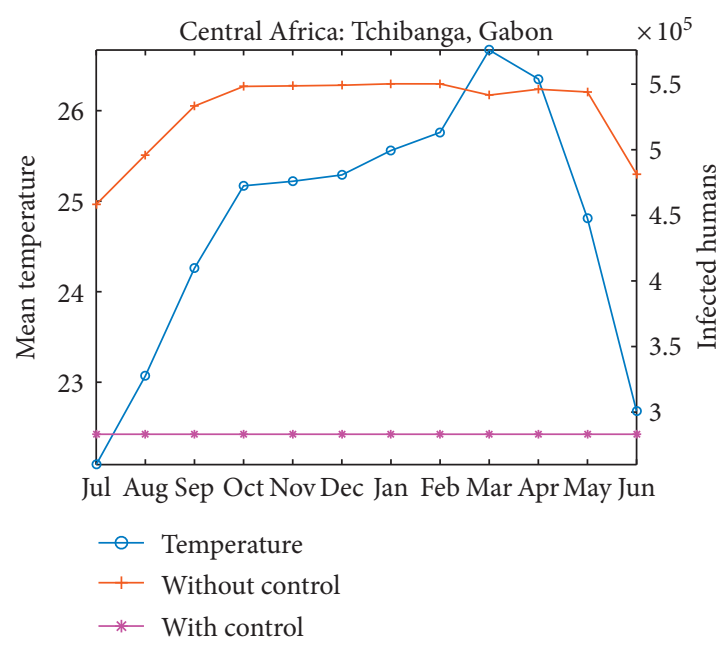

(c)

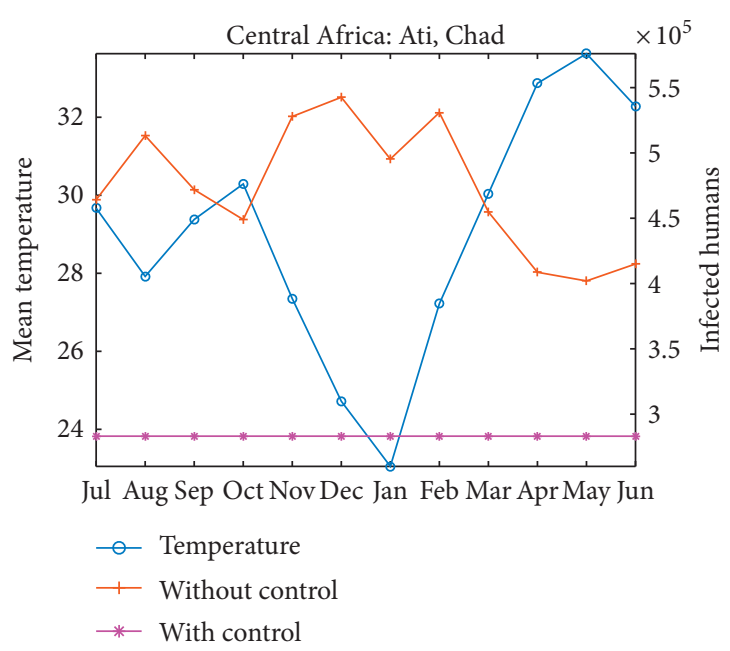

(e)

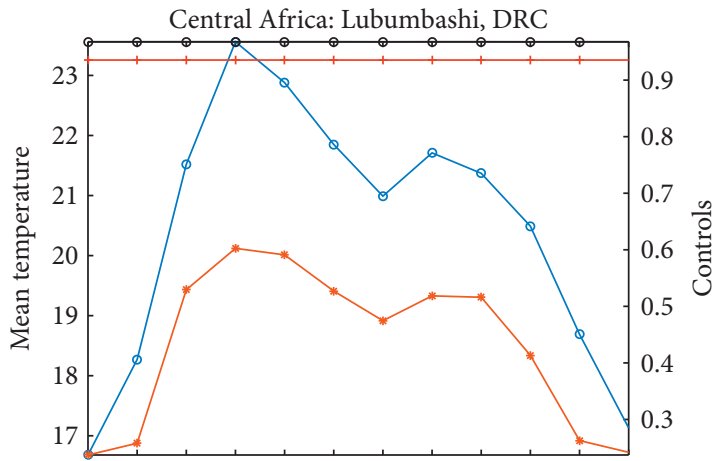

Jul Aug Sep Oct Nov Dec Jan Feb Mar Apr May Jun

$$
\begin{array}{ll}
- \text { Temperature } & \rightarrow \text { Control } U_{V} \\
- \text { Control } U_{L} & * \text { Control } U_{H}
\end{array}
$$

(b)

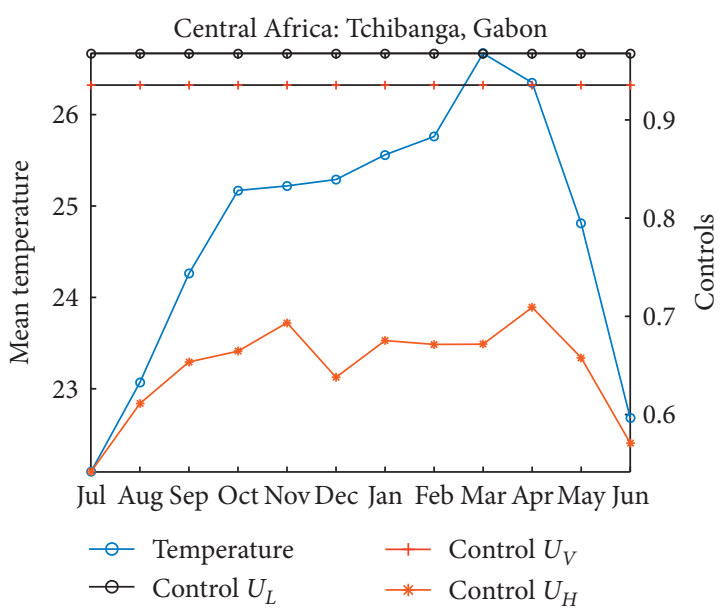

(d)

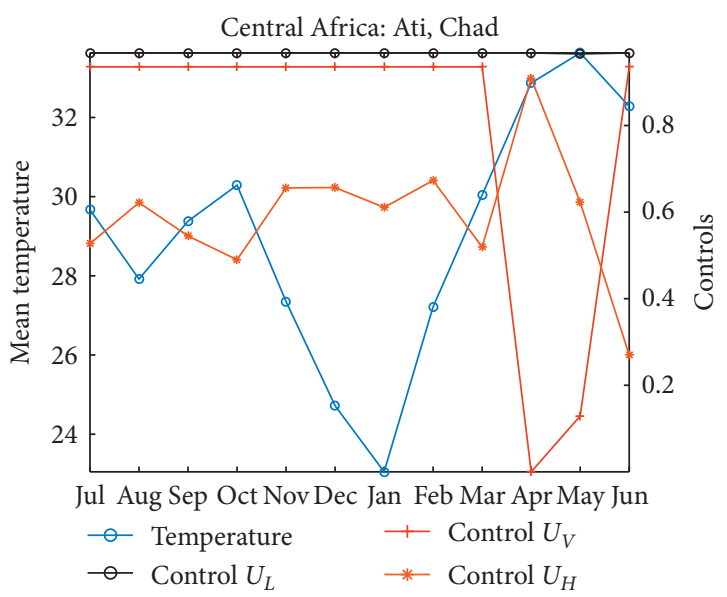

(f)

Figure 6: Simulations of the model (2) as a function of time: (a, c, e) the monthly total number of infected humans without and with controls and (b, d, f) optimal controls $u_{H}(t), u_{L}(t)$, and $u_{V}(t)$, in Lubumbashi, DRC; Tchibanga, Gabon; and Ati, Chad; all Central Africa. The mean monthly temperature is plotted in blue lines and is superimposed onto each figure. 


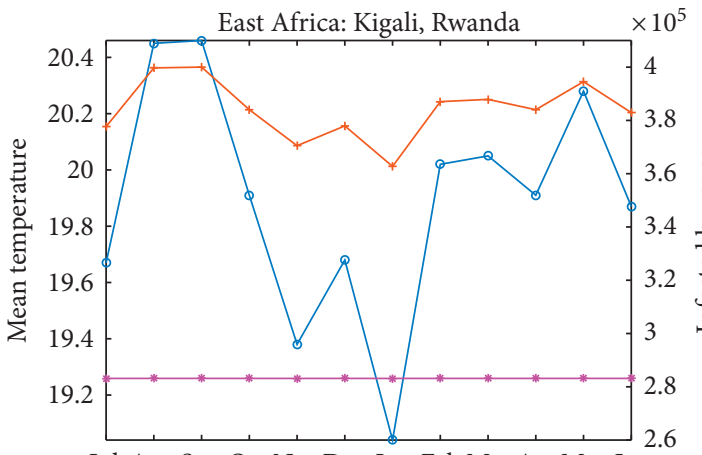

Jul Aug Sep Oct NovDec Jan Feb Mar Apr May Jun

$\multimap$ Temperature

+ Without control

$\rightarrow$ With control

(a)

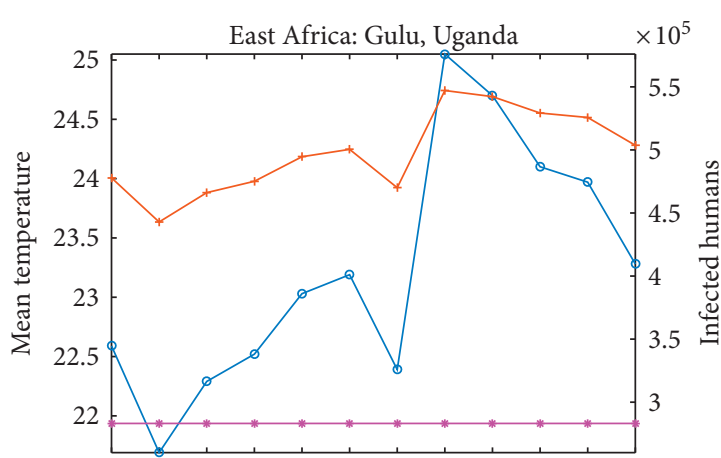

Jul Aug Sep Oct NovDec Jan Feb Mar AprMay Jun

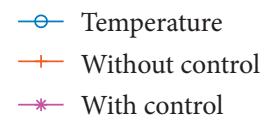

(c)

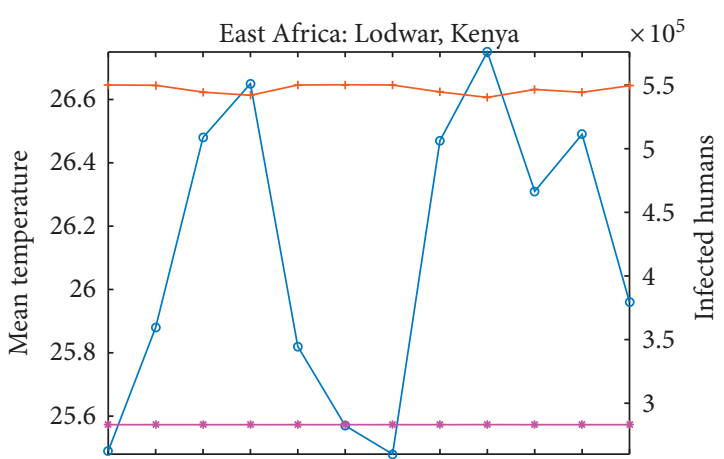

Jul Aug Sep Oct NovDec Jan Feb Mar Apr May Jun

$$
\begin{aligned}
& - \text { Temperature } \\
& + \text { Without control } \\
& \rightarrow \text { With control }
\end{aligned}
$$

(e)

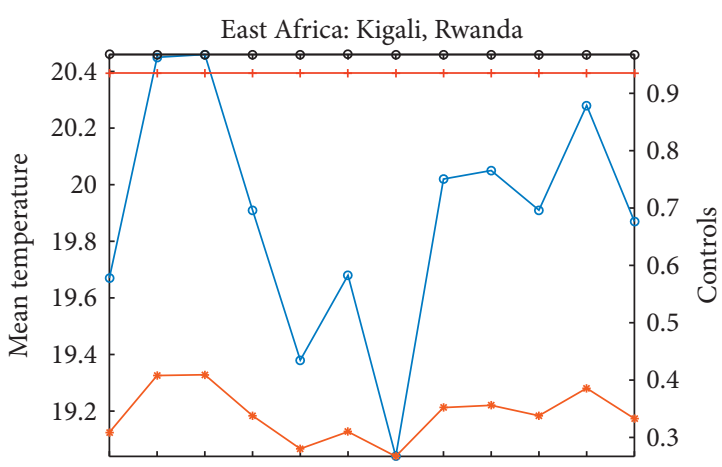

Jul Aug Sep Oct Nov Dec Jan Feb Mar Apr May Jun

$$
\begin{array}{ll}
\odot \text { Temperature } & + \text { Control } U_{V} \\
- \text { Control } U_{L} & * \text { Control } U_{H}
\end{array}
$$

(b)

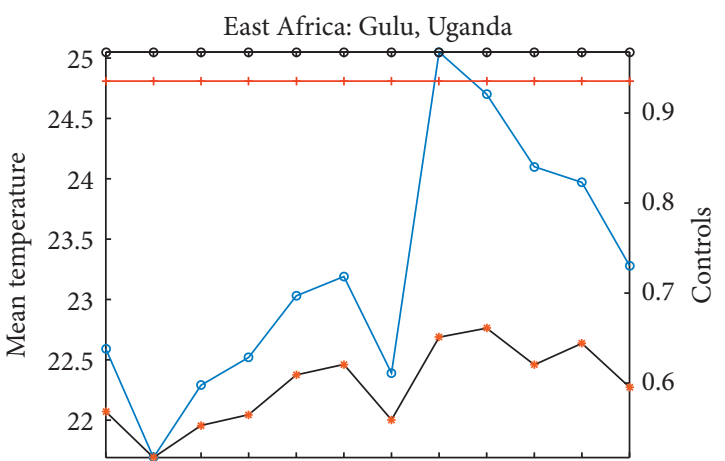

Jul Aug Sep Oct NovDec Jan Feb Mar AprMay Jun

$$
\begin{array}{ll}
- \text { Temperature } & \rightarrow \text { Control } U_{V} \\
- \text { Control } U_{L} & \rightarrow \text { Control } U_{H}
\end{array}
$$

(d)

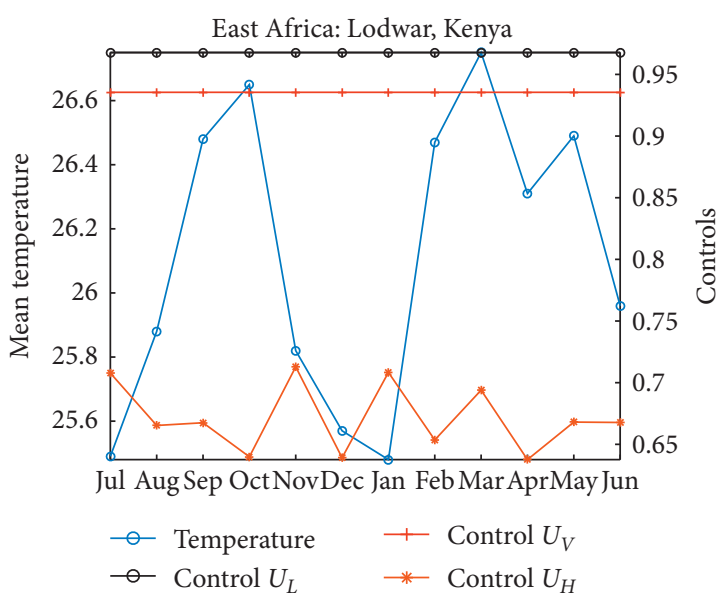

(f)

FIGURE 7: Simulations of the model (2) as a function of time: (a, c, e) the monthly total number of infected humans without and with controls and (b, d, f) optimal controls $u_{H}(t), u_{L}(t)$, and $u_{V}(t)$, in Kigali, Rwanda; Gulu, Uganda; and Lodwar, Kenya; in East Africa. The mean monthly temperature is plotted in blue lines and is superimposed onto each figure. 


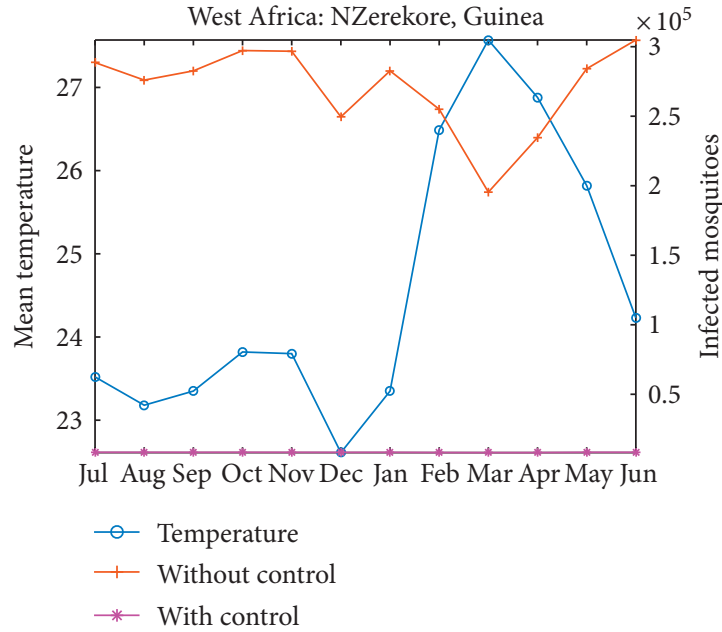

(a)

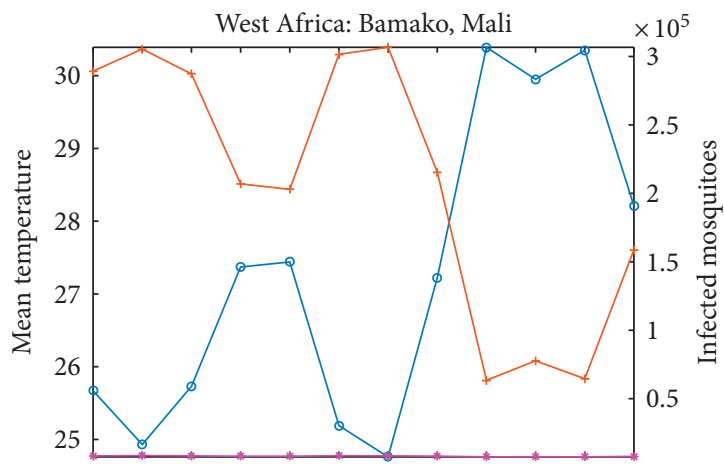

Jul Aug Sep Oct Nov Dec Jan Feb Mar Apr May Jun

$$
\begin{aligned}
& -\odot \text { Temperature } \\
& + \text { Without control } \\
& + \text { * With control }
\end{aligned}
$$

(c)

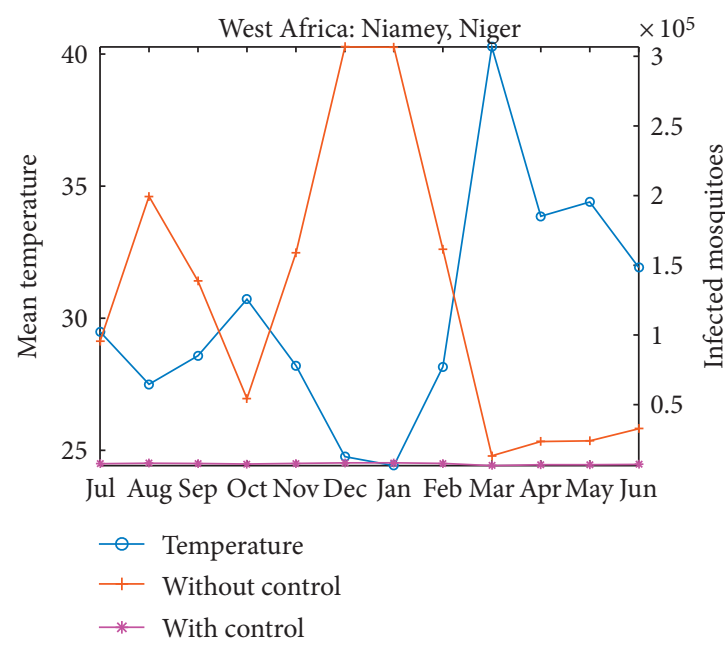

(e)

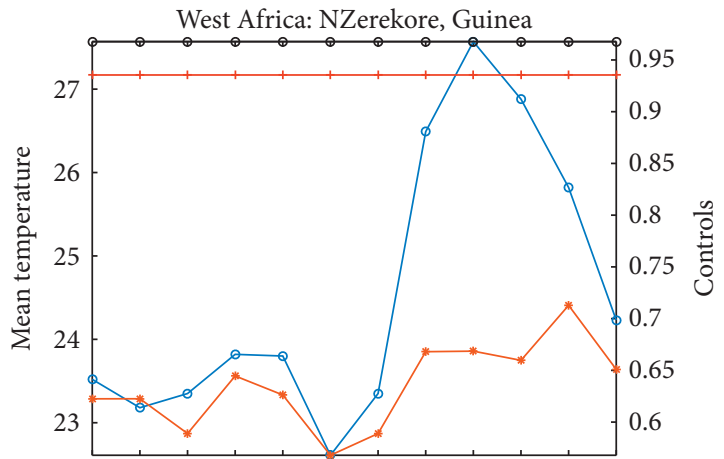

Jul Aug Sep Oct NovDec Jan Feb Mar Apr May Jun

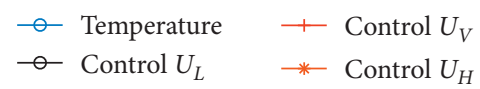

(b)

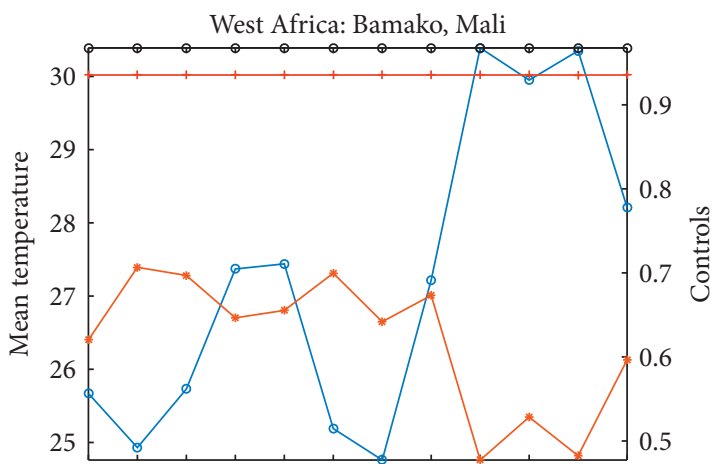

Jul Aug Sep Oct Nov Dec Jan Feb Mar Apr May Jun

$$
\begin{array}{ll}
- \text { Temperature } & + \text { Control } U_{V} \\
- \text { Control } U_{L} & \rightarrow \text { Control } U_{H}
\end{array}
$$

(d)

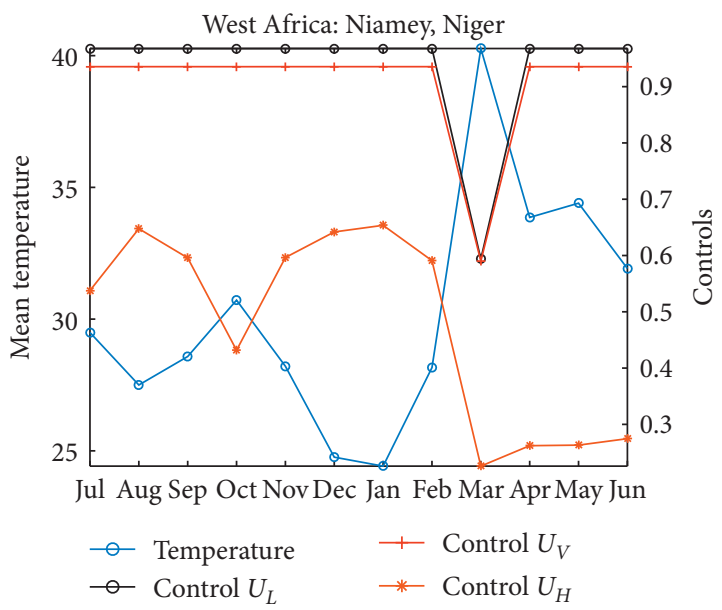

(f)

FIGURE 8: Simulations of the model (2) as a function of time: (a, c, e) the monthly total number of infected mosquitoes without and with control and (b, d, f) optimal controls $u_{H}(t), u_{L}(t)$, and $u_{V}(t)$, in NZerekore, Guinea; Bamako, Mali; and Niamey, Niger; in West Africa. The mean monthly temperature is plotted in blue lines and is superimposed onto each figure. 


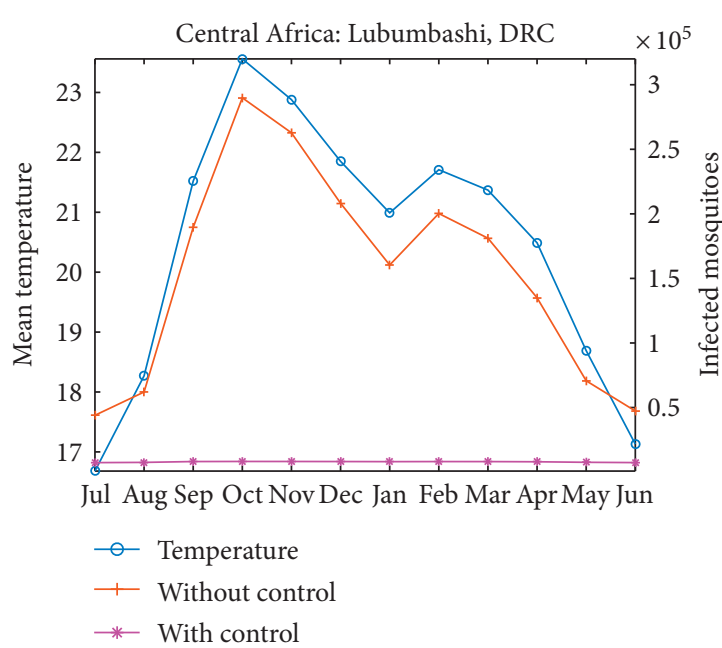

(a)

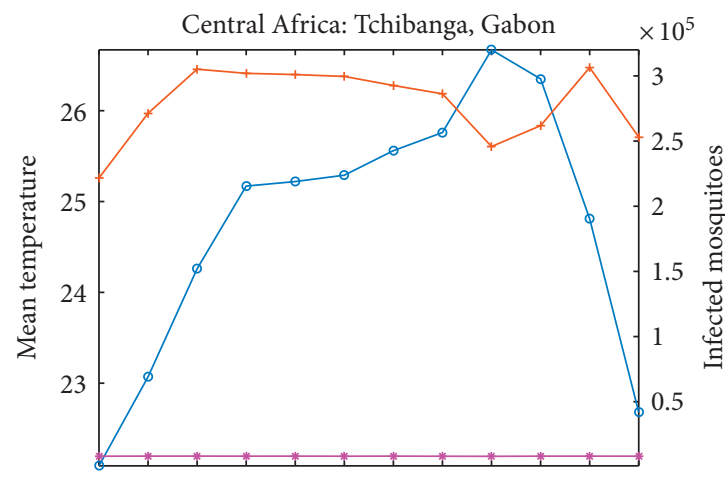

Jul Aug Sep Oct Nov Dec Jan Feb Mar Apr May Jun

$$
\begin{aligned}
& - \text { Temperature } \\
& + \text { Without control } \\
& * \text { With control }
\end{aligned}
$$

(c)

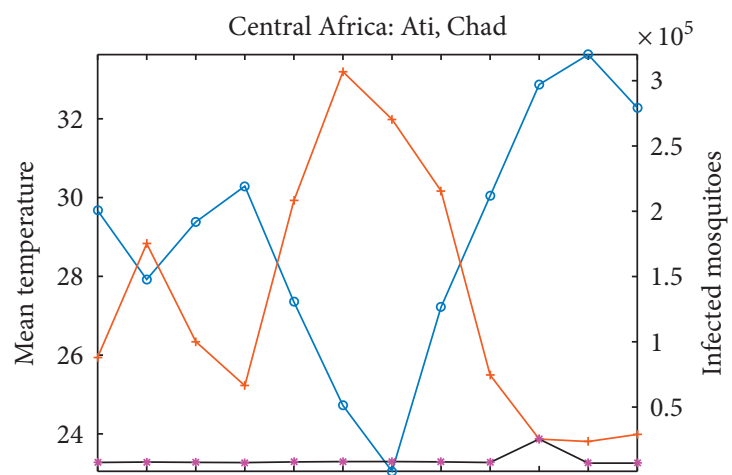

Jul Aug Sep Oct Nov Dec Jan Feb Mar Apr May Jun

$$
\begin{aligned}
& - \text { Temperature } \\
& + \text { Without control } \\
& - \text { - With control }
\end{aligned}
$$

(e)

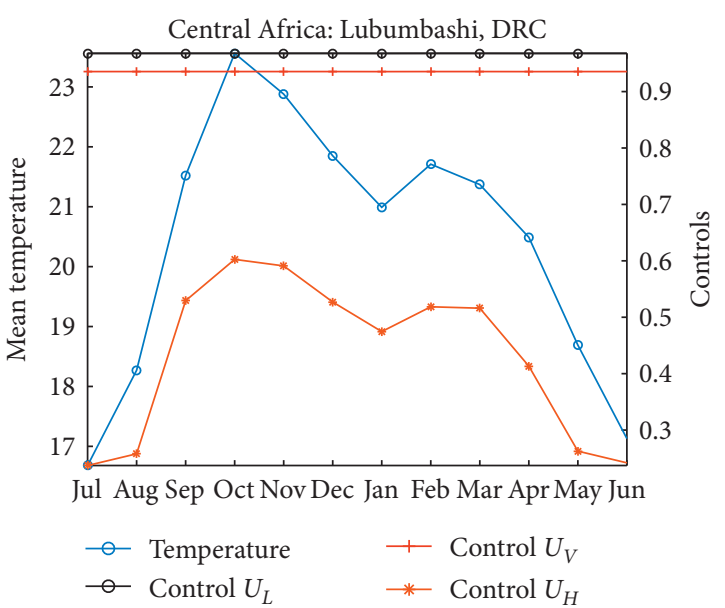

(b)

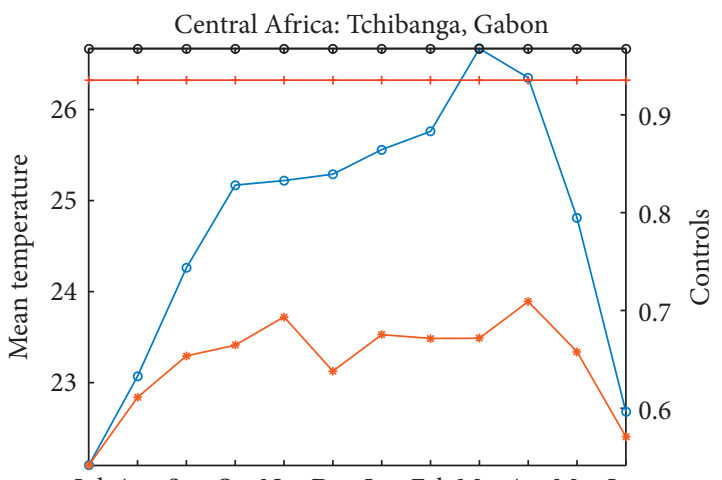

Jul Aug Sep Oct Nov Dec Jan Feb Mar Apr May Jun

$$
\begin{array}{ll}
\multimap \text { Temperature } & \rightarrow \text { Control } U_{V} \\
\odot \text { Control } U_{L} & * \operatorname{Control} U_{H}
\end{array}
$$

(d)

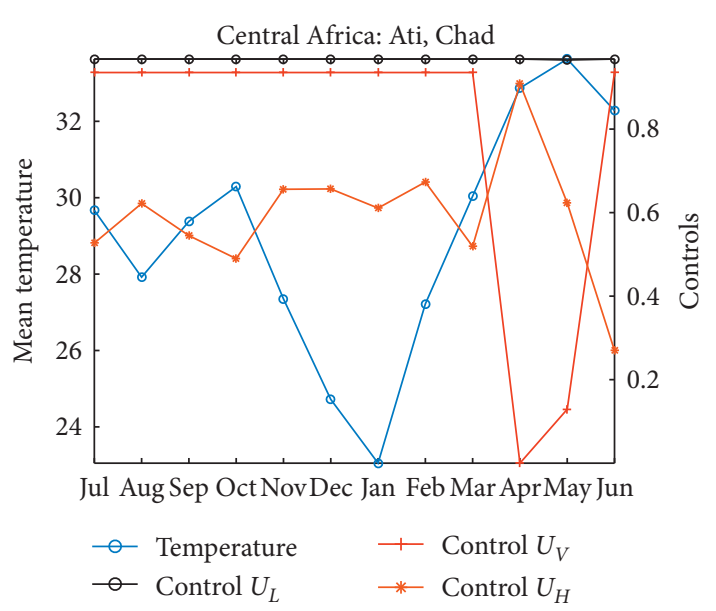

(f)

FIGURE 9: Simulations of the model (2) as a function of time: (a, c, e) the monthly total number of infected mosquitoes without and with control and (b, d, f) optimal controls $u_{H}(t), u_{L}(t)$, and $u_{V}(t)$, in Lubumbashi, DRC; Tchibanga, Gabon; and Ati, Chad; in Central Africa. The mean monthly temperature is plotted in blue lines and is superimposed onto each figure. 


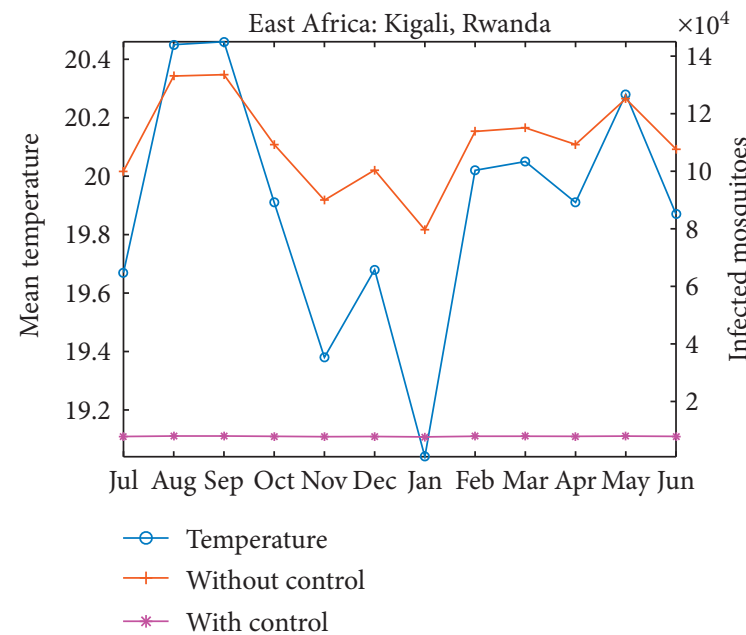

(a)

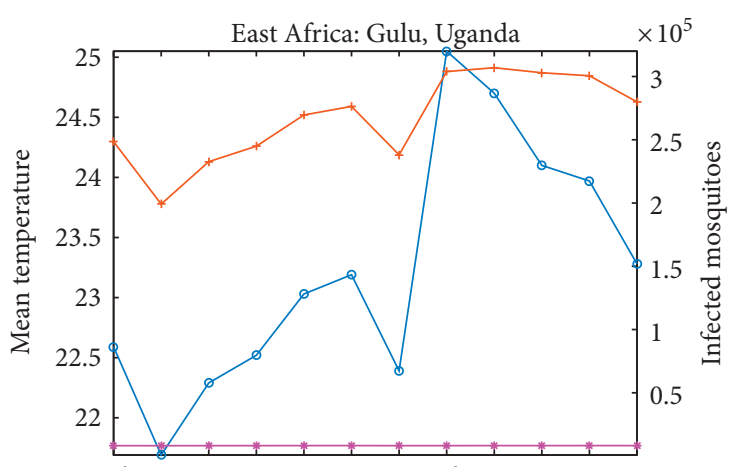

Jul Aug Sep Oct NovDec Jan Feb Mar Apr May Jun

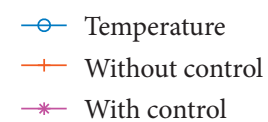

(c)

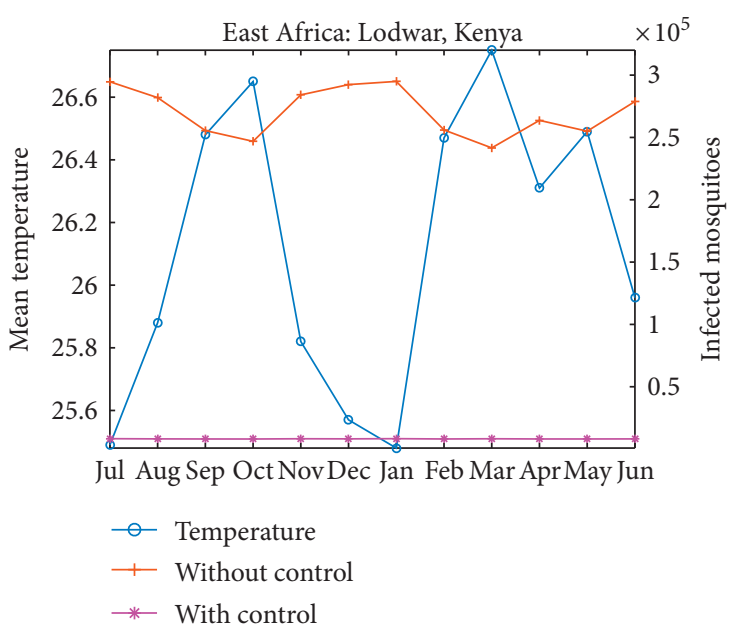

(e)

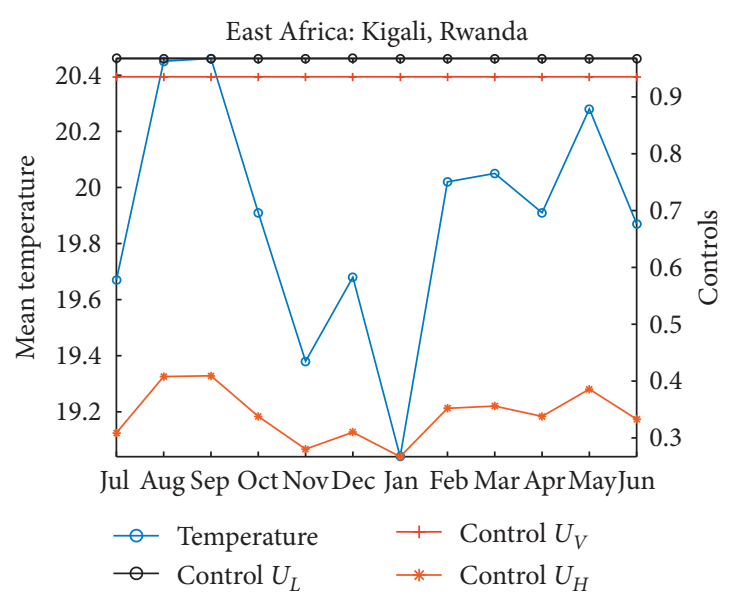

(b)

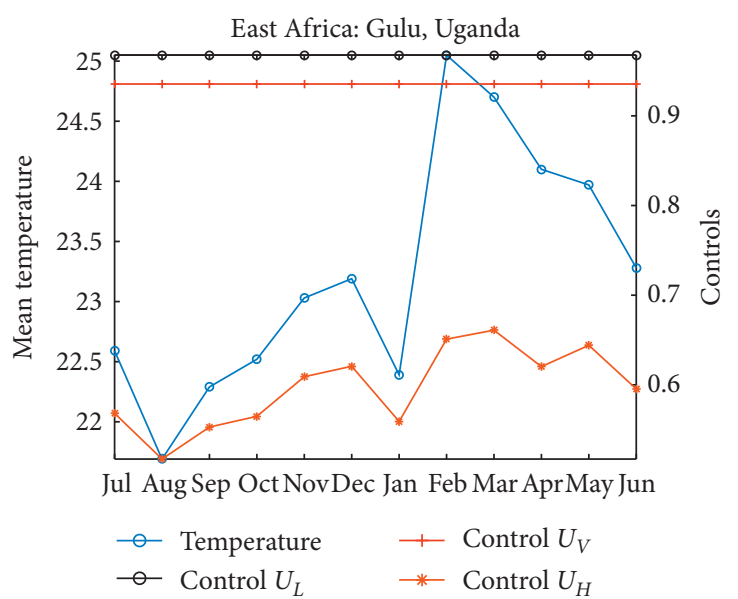

(d)

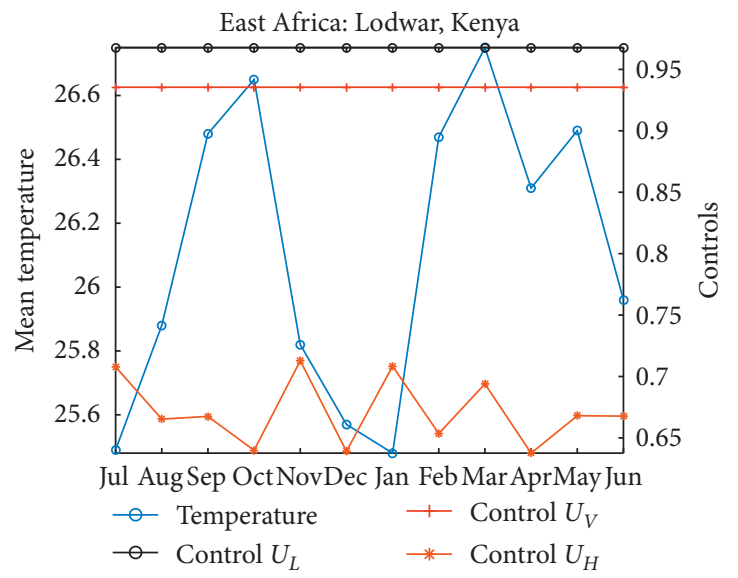

(f)

FIGURE 10: Simulations of the model (2) as a function of time: (a, c, e) the monthly total number of infected mosquitoes without and with control and (b, d, f) optimal controls $u_{H}(t), u_{L}(t)$, and $u_{V}(t)$, in Kigali, Rwanda; Gulu, Uganda; and Lodwar, Kenya; in East Africa. The mean monthly temperature is plotted in blue lines and is superimposed onto each figure. 
dependent controls $u_{L}(t)$ and $u_{V}(t)$ are close to the upper bound for all temperature values in the three cities of West Africa (Figures 5(b), 5(d), and 5(f)). The time-dependent control $u_{H}(t)$ starts relatively low for low temperatures and is relatively high for high temperatures. It should be noted that the solution profile for $u_{H}(t)$ varies from about 0.25 to 0.7 as the temperature gradient varies across West Africa. The control $u_{H}(t)$ is low at about 0.25 for low temperatures and about 0.7 for high temperatures.

In Central Africa, the application of the control measures leads to fewer infected individuals (see the infected humans in Figure 6). The average time-dependent controls $u_{L}(t)$ and $u_{V}(t)$ are all close to the upper bound for all temperature values in the three cities of Central Africa, see Figures 6(b), $6(\mathrm{~d})$, and $6(\mathrm{f})$. The time-dependent control $u_{H}(t)$ varies from about 0.3 to 0.8 as the temperature gradient varies across Central Africa. Similar to what was observed in West Africa, the control $u_{H}(t)$ is low at 0.3 for low temperatures and is relatively high at 0.8 for high temperatures.

Also, in East Africa, there are fewer infected individuals with the application of the control measures (see the infected humans in Figure 7). The average time-dependent control $u_{H}(t)$ varies from about 0.3 to 0.7 , while the controls $u_{L}(t)$ and $u_{V}(t)$ are close to the upper bound as the temperature gradient varies across the three cities of East Africa, see Figures $7(\mathrm{~b}), 7(\mathrm{~d})$, and $7(\mathrm{f})$. The time-dependent control $u_{H}(t)$ starts relatively low at 0.3 for low temperature and is relatively high at 0.7 for high temperature.

\subsection{Control in Temperature Ranges Suitable for Mosquitoes}

4.2.1. Suitable Temperature Ranges: Infected Human Profiles. Next, we explore closely the optimal control strategies in the temperature range suitable for mosquitoes identified in the Mean Monthly Temperature: Other Cities Infected Human Profiles section [2]. In West Africa, the temperature range is $\left[22.61^{\circ} \mathrm{C}, 28.58^{\circ} \mathrm{C}\right]$, and in Central Africa, the temperature is $\left[16.68^{\circ} \mathrm{C}, 27.92^{\circ} \mathrm{C}\right]$. The temperature range in East Africa is $\left[19.04^{\circ} \mathrm{C}, 26.75^{\circ} \mathrm{C}\right]$, and in South Africa, the range is $\left[16.7^{\circ} \mathrm{C}, 25.3^{\circ} \mathrm{C}\right]$.

We observed, in Figure 11(a) in the absence of control, an increase in the total number of infected humans in West Africa until the midtemperature of $25.59^{\circ} \mathrm{C}$ before decreasing at a higher temperature. However, in Central, East, and South Africa, the total number of infected humans increases with increasing temperature (Figures 11(c), 11(e), and $11(\mathrm{~g}))$. The application of the control strategies reduced the number of infected humans. Similar dynamics are observed for mosquitoes, and the plots are given in Figure 12.

We observed in Figures 11(b), 11(d), 11(f), and 11(h) that the average time-dependent controls $u_{L}(t)$ and $u_{V}(t)$ are close to the upper bound for all temperature values in all the four regions. In West Africa, the average control $u_{H}(t)$ increases until the midtemperature of $25.59^{\circ} \mathrm{C}$ before decreasing at a higher temperature, while in East, Central, and South Africa, the time-dependent control $u_{H}(t)$ increases with increasing temperatures.
The optimal control strategies with temperature suggest that very high levels of larvicides and adulticides should be used. These high levels of insecticide, particularly from the scale-up use of insecticide-treated bednets, and indoor spraying have been observed to lead to the development of resistance mosquito $[59,60]$. This widespread use of insecticide-treated bednets and indoor spraying has been largely successful in reducing the incidence of malaria across sub-Sharan Africa [61]. However, the widespread use of these has increased the pressure on mosquitoes to evolve resistance to these commonly used pyrethroid insecticides $[59,62]$.

Using sequencing techniques and genetic analyses, Barnes et al. [59] carried out a continent-wide population structure study of Anopheles funestus, a major African malaria mosquito. They located a gene region that has allowed mosquitoes to evolutionarily adapt to insecticides by enabling them to break down insecticides [59]. This resistance form of this gene has now swept through mosquito populations in southern Africa to become almost universal [60].

Coupling our result from the optimal control of insecticide mosquitoes and the findings of Barnes et al. [59], we develop a model to study the control of malaria in the presence of temperature variation and insecticide-resistant mosquitoes.

\section{Malaria Model with Insecticide- Resistant Mosquitoes}

Following the results obtained in the Optimal Control with Mean Monthly Temperatures section involving the high levels of adulticides and larvacides controls, we extend the simple malaria model used in Agusto et al. [2] to include both insecticide-sensitive and insecticide-resistant mosquitoes. The human population, of size $N_{H}(t)$, is split into mutually exclusive subpopulations of individuals who are susceptible $\left(S_{H}(t)\right)$, exposed $\left(E_{H}(t)\right)$, infectious $\left(I_{H}(t)\right)$, and recovered from the infection $\left(R_{H}(t)\right)$ so that

$$
N_{H}(t)=S_{H}(t)+E_{H}(t)+I_{H}(t)+R_{H}(t) .
$$

Similarly, the total mosquito population size at time $t$, denoted by $N_{V}(t)$, is subdivided into immature mosquitoes (eggs, larvae, and pupae) $\left(L_{V}(t)\right)$, susceptible insecticidesensitive and insecticide-resistant mosquitoes $\left(S_{V_{S}}(t), S_{V_{R}}(t)\right)$, exposed insecticide-sensitive and insecticide-resistant mosquitoes $\left(E_{V_{S}}(t), E_{V_{R}}(t)\right)$, and infectious insecticide-sensitive and insecticide-resistant mosquitoes $\left(I_{V_{S}}(t), I_{V_{R}}(t)\right)$. We assume for simplicity that the mosquitoes differentiate into insecticide-sensitive and insecticide-resistant mosquitoes only at the adult stage.

Thus, the simple model is given by the following deterministic, nonautonomous, system of nonlinear differential equations (where the dot notation, as previously stated, denotes differentiation with respect to time $t$ and $T$ and $\widehat{T}=T+\delta_{T}$ denote air and water temperature, respectively): 


$$
\begin{aligned}
& \dot{S_{H}}(t)=\Pi_{H}+\omega_{1} R_{H}(t)-\lambda_{H_{S}}\left(T, I_{V_{S}}, N_{H}\right) S_{H}(t)-\lambda_{H_{R}}\left(T, I_{V_{R}}, N_{H}\right) S_{H}(t)-\mu_{H} S_{H}(t), \\
& \dot{E_{H}}(t)=\lambda_{H_{S}}\left(T, I_{V_{S}}, N_{H}\right) S_{H}(t)+\lambda_{H_{R}}\left(T, I_{V_{R}}, N_{H}\right) S_{H}(t)-\left(\sigma_{H}+\mu_{H}\right) E_{H}(t), \\
& \dot{I_{H}}(t)=\sigma_{H} E_{H}(t)-\left(\gamma_{H}+\mu_{H}+\delta_{H}\right) I_{H}(t), \\
& \dot{R_{H}}(t)=\gamma_{H} I_{H}(t)-\left(\omega_{H}+\mu_{H}\right) R_{H}(t), \\
& \dot{L_{V}}(t)=\phi_{V}(T)\left[1-\frac{L_{V}(t)}{K_{V}}\right]\left[S_{V_{S}}(t)+E_{V_{S}}(t)+I_{V_{S}}(t)+S_{V_{R}}(t)+E_{V_{R}}(t)+I_{V_{R}}(t)\right] \\
& \quad-\left[\theta_{V}(\widehat{T})+\mu_{L}(\widehat{T})\right] L_{V}(t), \\
& \dot{S_{V_{S}}}(t)=f \theta_{V}(\widehat{T}) L_{V}(t)-\lambda_{V_{S}}\left(T, N_{H}\right) S_{V_{S}}(t)-\mu_{V_{S}}(T) S_{V_{S}}(t), \\
& \dot{E_{V_{S}}}(t)=\lambda_{V_{S}}\left(T, N_{H}\right) S_{V_{S}}(t)-\left[\sigma_{V_{S}}(T)+\mu_{V_{S}}(T)\right] E_{V_{S}}(t), \\
& \dot{I_{V_{S}}}(t)=\sigma_{V_{S}}(T) E_{V_{S}}(t)-\mu_{V_{S}}(T) I_{V_{S}}(t), \\
& \dot{S_{V_{R}}}(t)=(1-f) \theta_{V}(\widehat{T}) L_{V}(t)-\lambda_{V_{R}}\left(T, N_{H}\right) S_{V_{R}}(t)-\mu_{V_{R}}(T) S_{V_{R}}(t), \\
& \dot{E_{V_{R}}}(t)=\lambda_{V_{R}}\left(T, N_{H}\right) S_{V_{R}}(t)-\left[\sigma_{V_{R}}(T)+\mu_{V_{R}}(T)\right] E_{V_{R}}(t), \\
& \dot{I_{V_{R}}}(t)=\sigma_{V_{R}}(T) E_{V_{R}}(t)-\mu_{V_{R}}(T) I_{V_{R}}(t),
\end{aligned}
$$

where

$$
\begin{aligned}
\lambda_{H_{S}}\left(T, I_{V_{S}}(t), N_{H}(t)\right) & =\frac{\beta_{H} b_{V}(T) I_{V_{S}}(t)}{N_{H}(t)}, \\
\lambda_{H_{R}}\left(T, I_{V_{R}}(t), N_{H}(t)\right) & =\frac{\eta_{H_{R}} \beta_{H} b_{V}(T) I_{V_{R}}(t)}{N_{H}(t)}, \\
\lambda_{V_{S}}\left(T, N_{H}\right) & =\frac{\beta_{V} b_{V}(T) I_{H}(t)}{N_{H}(t)}, \\
\lambda_{V_{R}}\left(T, N_{H}\right) & =\frac{\eta_{V_{R}} \beta_{V} b_{V}(T) I_{H}(t)}{N_{H}(t)} .
\end{aligned}
$$

Furthermore, we assume that $\sigma_{V_{R}}(T)=\eta_{R_{1}} \sigma_{V_{S}}(T)$ and $\mu_{V_{R}}(T)=\eta_{R_{2}} \mu_{V_{S}}(T)$. The parameters $\eta_{H_{R}}, \eta_{V_{R}}, \eta_{R_{1}}$, and $\eta_{R_{2}}$ are modification parameters indicating the difference between the insecticide-sensitive and insecticide-resistant-related functions. We have assumed that $\eta_{H_{R}}=\eta_{V_{R}}=\eta_{R_{1}}=1$; indicating that the mosquitoes' insecticide-resistant gene has no impact on mosquito biting rate and transmission probability, nor does it affect the parasite developmental rate. However, there are indications on the contrary [63-65]. We make these assumptions for simplicity, and without loss of generality, we believe that there will be no wide deviation from the conclusions of this study if we had incorporated these features. Furthermore, we suppose the resistance mosquitoes are twice likely to live longer than the sensitive mosquitoes; hence, we set $\eta_{R_{2}}=0.5$. However, infected insecticide-resistant mosquitoes are more susceptible to insecticide [64, 66]. Note, $\sigma_{V_{S}}(T)$ and $\mu_{V_{S}}(T)$ are given by the same functions as those in equations (6) and (8), respectively.

The flow diagram of the model is depicted in Figure 13, the associated state variables are described in Table 4, and parameters are described in Table 1.

5.1. Optimal Control Problem with Resistance Mosquitoes. The goal in this section is to minimize the objective function defined as

$$
\begin{aligned}
J_{R}\left(u_{H}(t), u_{L}(t), u_{V}(t)\right)= & \int_{0}^{t_{f}}\left[A_{1} I_{H}+B_{1} L_{V}+B_{2}\left(S_{V_{S}}+S_{V_{R}}\right)+B_{3}\left(E_{V_{S}}+E_{V_{R}}\right)\right. \\
& \left.+B_{4}\left(I_{V_{S}}+I_{V_{R}}\right)+C_{1} u_{H}+\varepsilon C_{2} u_{H}^{2}+C_{3} u_{L}+\varepsilon C_{4} u_{L}^{2}+C_{5} u_{V}+\varepsilon C_{6} u_{V}^{2}\right] \mathrm{d} t,
\end{aligned}
$$

that is, we seek to find the optimal controls $u_{H}^{*}, u_{L}^{*}$, and $u_{V}^{*}$, such that

$$
J_{R}\left(u_{H}^{*}, u_{L}^{*}, u_{V}^{*}\right)=\min _{u}\left\{J_{R}\left(u_{H}, u_{L}, u_{V}\right)\right\} .
$$

The optimal control solutions $\left(u_{H}(t), u_{L}(t), u_{V}(t)\right)$ can be characterized by following the approach in the Optimal Control Problems section. 


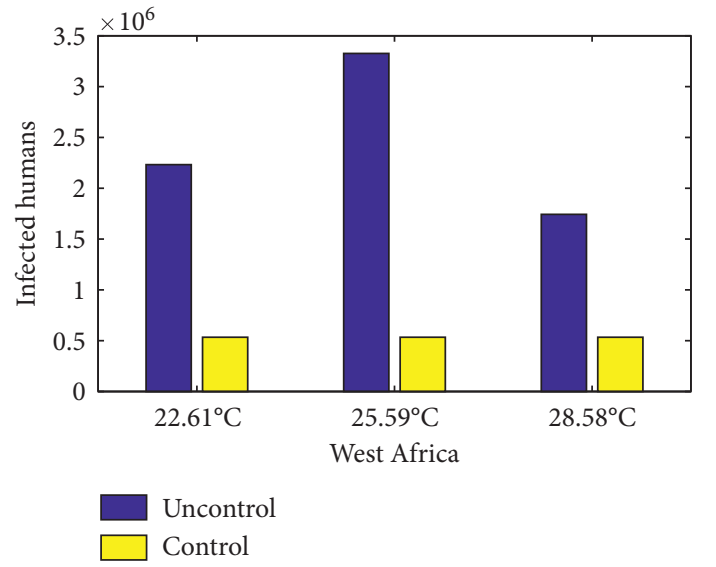

(a)

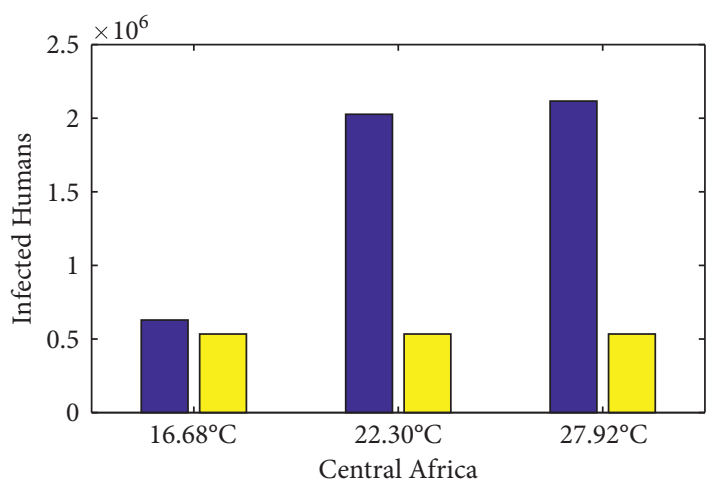

Uncontrol

Control

(c)

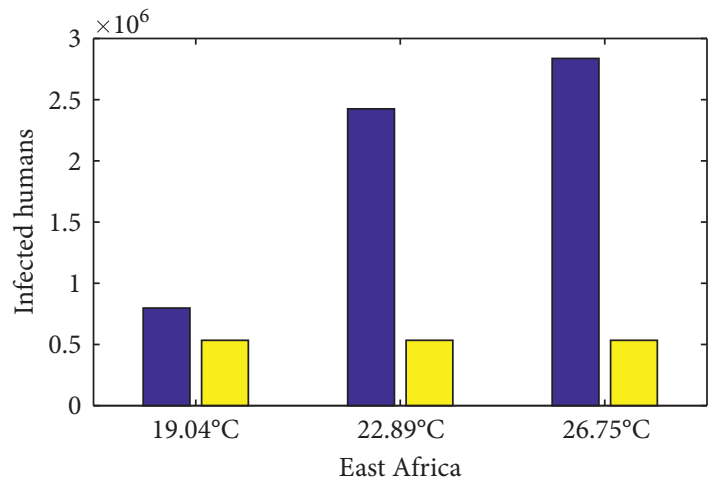

Uncontrol

Control
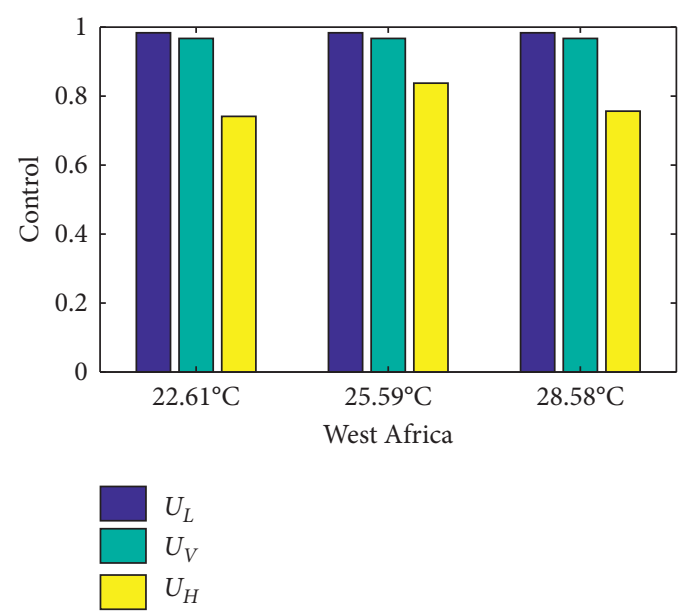

(b)

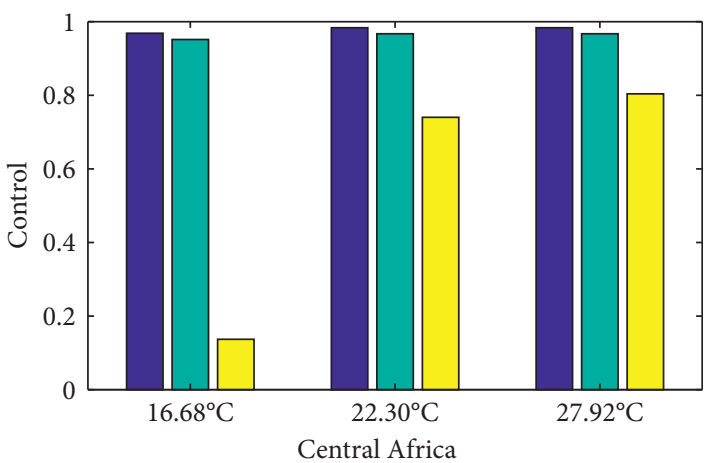

$\square U_{L}$
$\square U_{V}$
$\square U_{H}$

(d)
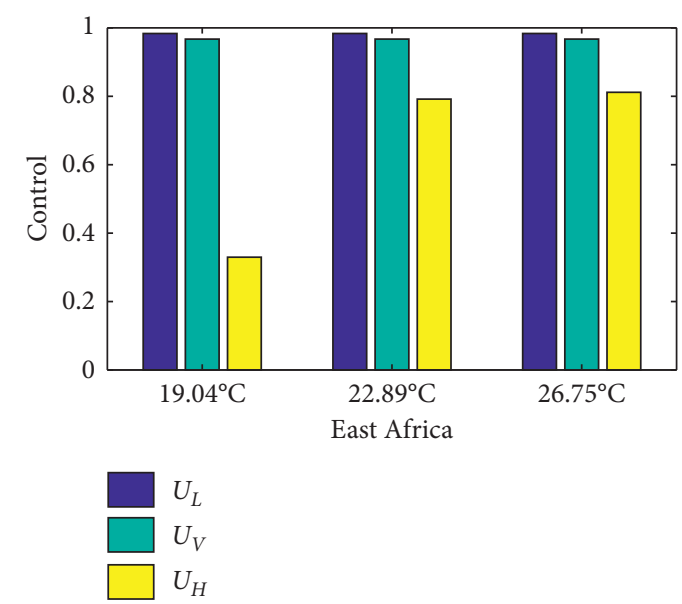

(e)

Figure 11: Continued. 


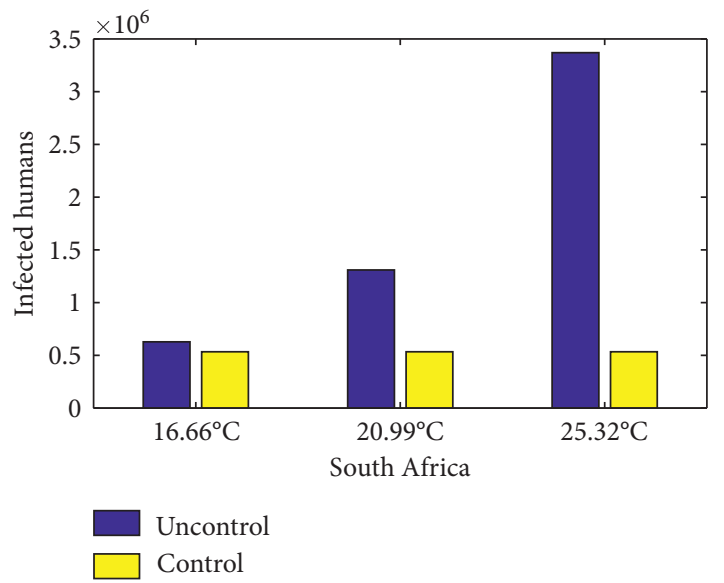

(g)

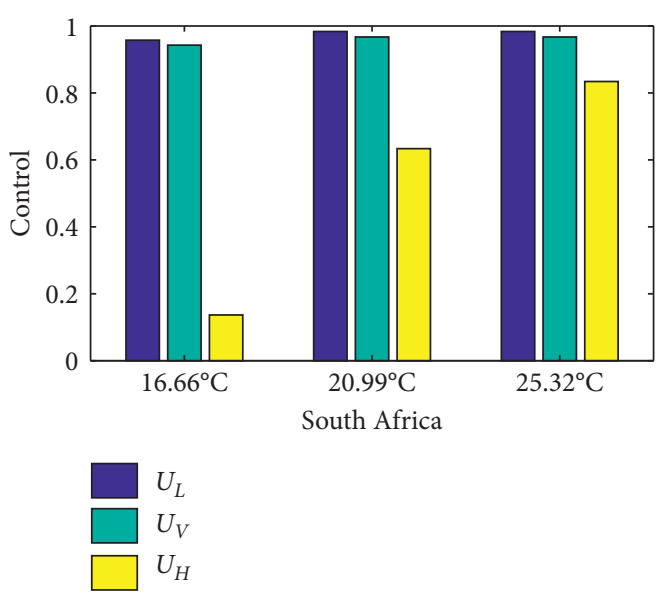

(h)

FIGURE 11: Simulations of the malaria model (2) as a function of time in temperature ranges suitable for mosquitoes: (a, c, e, g) the monthly total number of infected humans with control and (b, d, f, h) optimal controls $u_{H}(t), u_{L}(t)$, and $u_{V}(t)$, in West, Central, East, and South Africa.

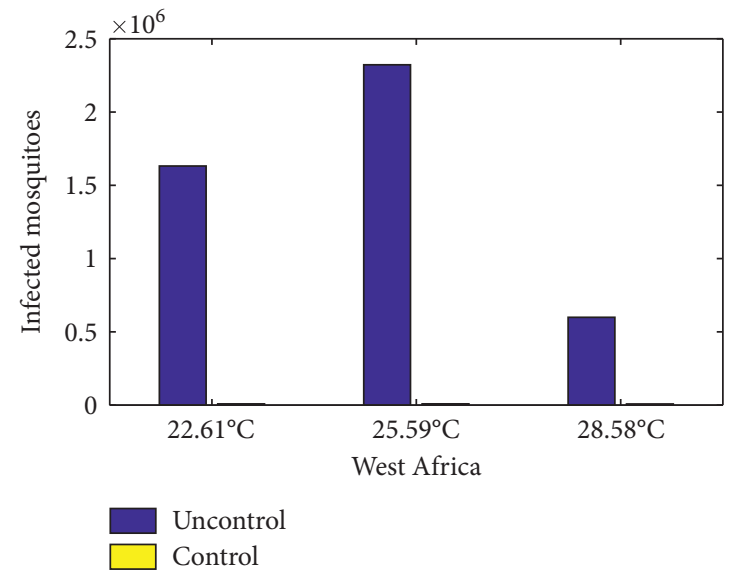

(a)

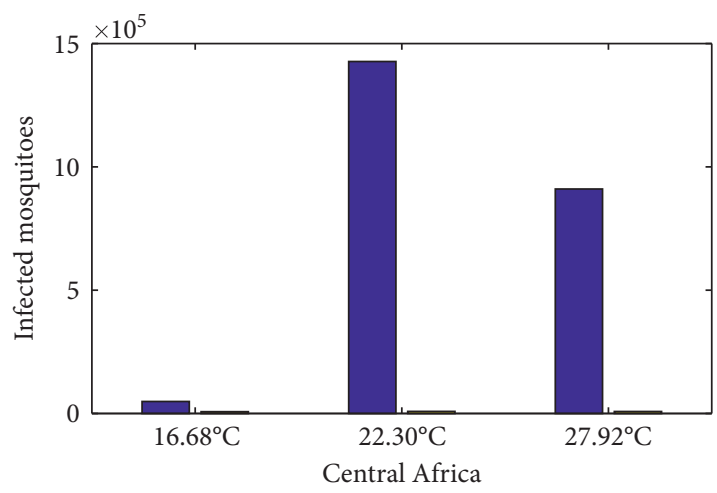

Uncontrol

Control

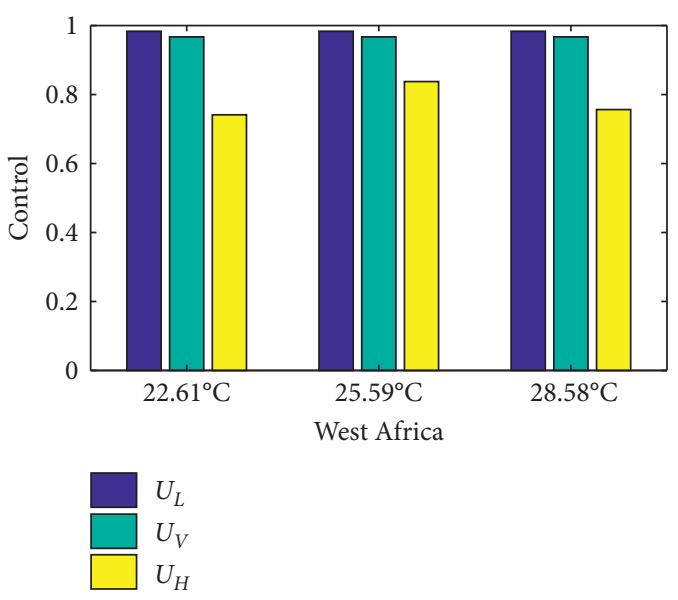

(b)

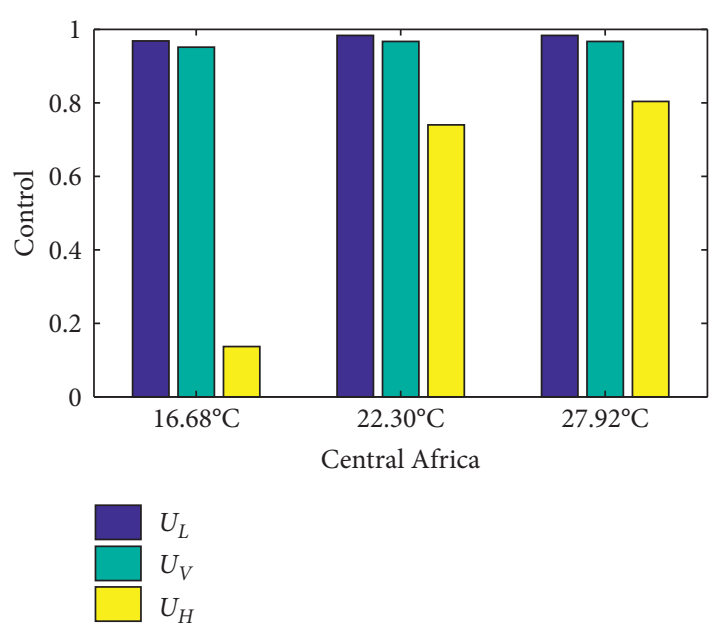

(d)

Figure 12: Continued. 


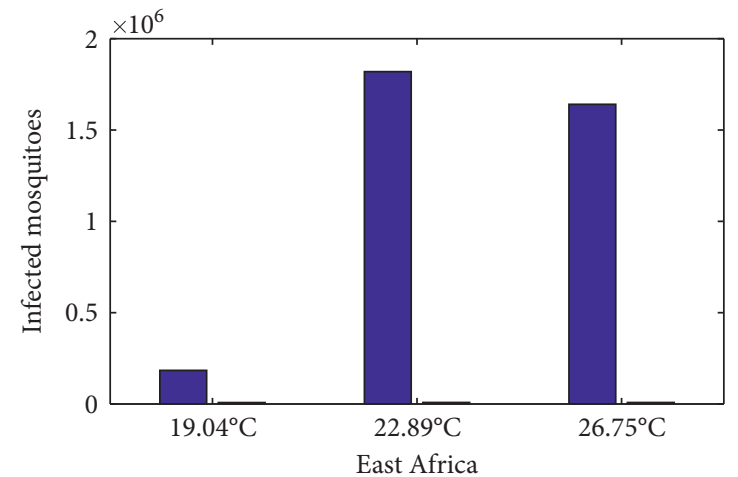

Uncontrol

Control

(e)

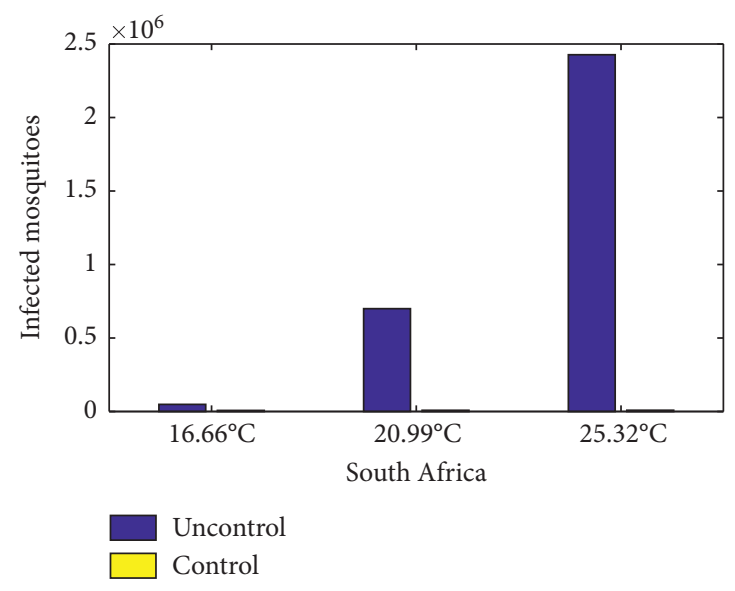

(g)
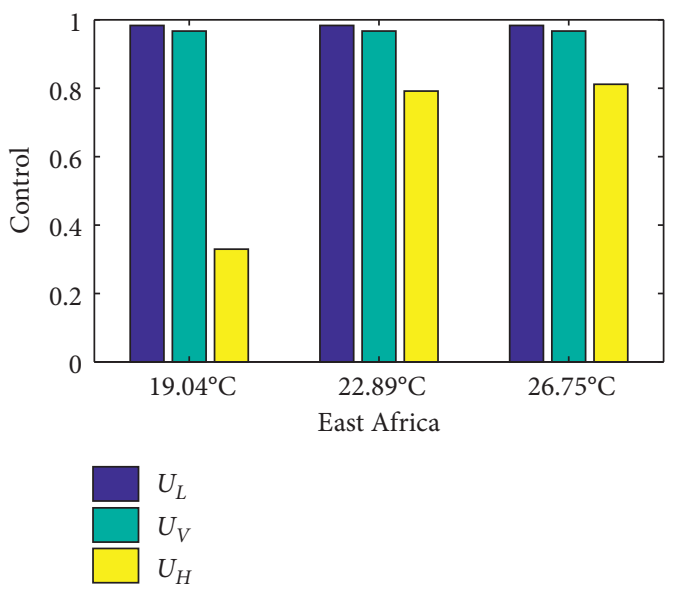

(f)

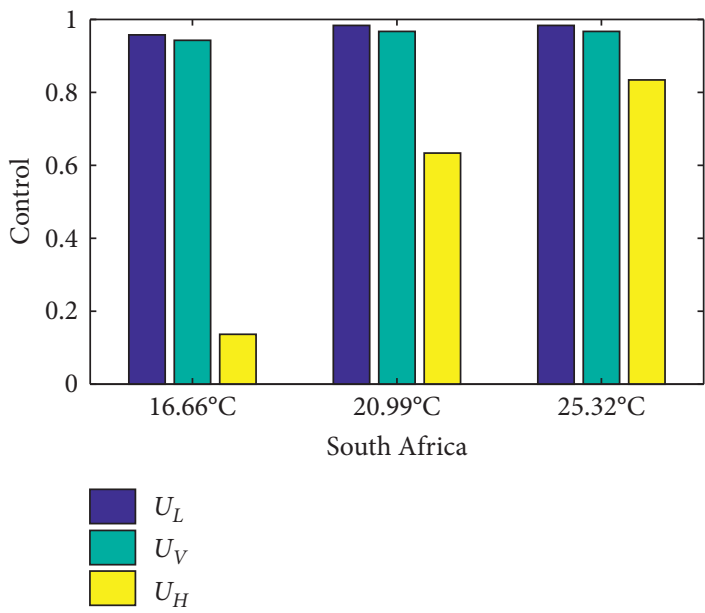

(h)

Figure 12: Simulations of the malaria model (2) as a function of time in the suitable temperature ranges. (a, c, e) The monthly total number of infected mosquitoes with control, and (b, d, f) optimal controls $u_{H}(t), u_{L}(t)$, and $u_{V}(t)$, in West, Central, East, Central, and South Africa.

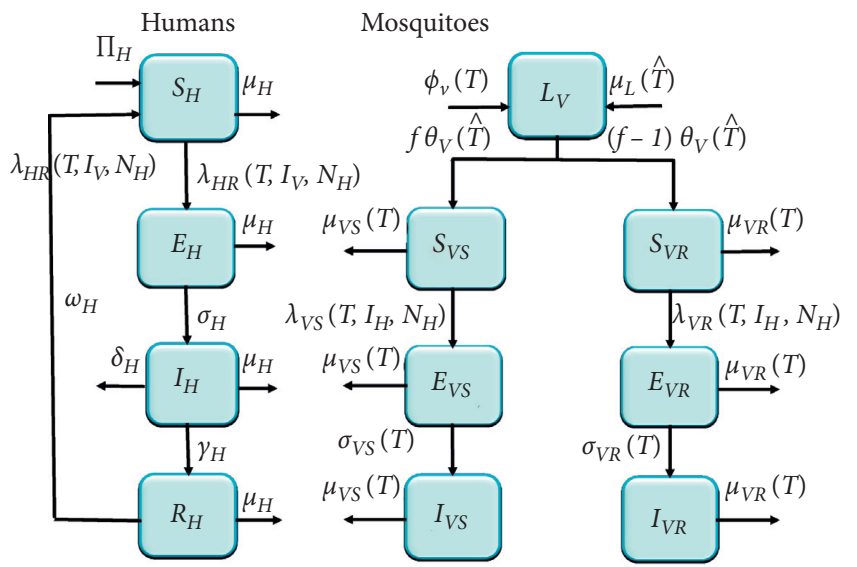

FIGURE 13: Flow diagram of the malaria-resistant model (21).
TABLE 4: Description of the variables of model (21).

\begin{tabular}{lc}
\hline Variable & Description \\
\hline$S_{H}(t)$ & Number of susceptible humans \\
$E_{H}(t)$ & Number of exposed humans \\
$I_{H}(t)$ & Number of infectious humans \\
$R_{H}(t)$ & Number of recovered humans \\
$L_{V}(t)$ & Number of immature (eggs, larva, and pupa stages) \\
$S_{V_{S}}(t)$ & vectors \\
$E_{V_{S}}(t)$ & Number of susceptible sensitive vectors \\
$I_{V_{S}}(t)$ & Number of exposed sensitive vectors \\
$S_{V_{R}}(t)$ & Number of infectious-sensitive vectors \\
$E_{V_{R}}(t)$ & Number of exposed resistance vectors \\
$I_{V_{R}}(t)$ & Number of infectious-resistant vectors \\
\hline
\end{tabular}


Our aim in this section is to apply optimal control theory to minimize the number of infected humans and mosquitoes (insecticide-sensitive and insecticide-resistant) in the temperature range suitable for mosquitoes in West, Central, East, and South Africa. As in the Control in Temperature Ranges Suitable for Mosquitoes section, we will use the mean total number of infected humans and mosquitoes to measure the impact of the mean level of controls $\left(u_{H}(t), u_{L}(t), u_{V}(t)\right)$ in these regions of sub-Sahara Africa. We will also investigate the impact of monocontrol strategies where the time-dependent controls $\left(u_{H}(t), u_{L}(t), u_{V}(t)\right)$ are applied one at a time.

\subsection{Resistance Mosquitoes: Temperature Ranges Suitable for Mosquitoes}

5.2.1. Control in Suitable Temperature Ranges: Infected Human Profiles. Next, we also explore the optimal control strategies in the temperature range suitable for mosquitoes identified in the Mean Monthly Temperature: Other Cities Infected Human Profiles section. In West Africa, the temperature range is $\left[22.16^{\circ} \mathrm{C}, 28.58^{\circ} \mathrm{C}\right]$, and in Central Africa, the temperature is $\left[16.68^{\circ} \mathrm{C}, 27.92^{\circ} \mathrm{C}\right]$. The temperature range in East Africa is $\left[19.04^{\circ} \mathrm{C}, 26.75^{\circ} \mathrm{C}\right]$, and in South Africa, the range is $\left[16.7^{\circ} \mathrm{C}, 25.3^{\circ} \mathrm{C}\right]$.

In Central, East, and South Africa, the mean total number of infected humans increases with increasing temperature (Figures 14(c), 14(e), and 14(g)). The application of the control strategies, depicted, in Figures 14(d), 14(f), and 14(h), reduced the mean number of infected humans, with the average level of the controls $u_{L}(t)$ and $u_{V}(t)$ close to the upper bound and the average control $u_{H}$ increases with the mean monthly temperature. Similar dynamics are observed in the mosquito population, and the plots are given in Figure 15.

5.3. Monocontrol Strategies. Next, we investigate the effect of monocontrol strategies on malaria transmission in subSaharan Africa using malaria insecticide-sensitive model (2) and insecticide-resistant model (21).

We considered the following two monocontrol strategies:

(i) Strategy A: controls $u_{L}(t)$ and $u_{V}(t)$-only while setting $u_{H}(t)=0$

(ii) Strategy B: control $u_{H}(t)$-only while setting $u_{L}(t)=u_{V}(t)=0$

We compare the solution profiles obtained from these strategies to the profile obtained from the control strategy when all the controls $u_{L}(t), u_{V}(t)$, and $u_{H}(t)$ are used (Figure 11).

5.3.1. Monocontrol Strategies for Sensitive Mosquitoes: Infected Human Profiles. Comparing the mean total number of infected humans in Figures 11 and 16, we observed a higher number of infected humans in the absence of control (Figure 11) and lower number with the application of the controls regardless of the control strategies (Figure 16). As in the previous sections, we calculated the average level for all the controls $u_{L}(t), u_{V}(t)$, and $u_{H}(t)$. We observed that Strategy B was relatively better than Strategy A in reducing the number of infected humans when we have insecticidesensitive mosquitoes only.

We observed in Figure 11(a) increase in the mean total number of infected humans in West Africa in the absence of control until the midtemperature of $25.59^{\circ} \mathrm{C}$ before decreasing at the highest temperature of $28.58^{\circ} \mathrm{C}$. The mean total number of infected humans was considerably reduced in the presence of control. Furthermore, in Figure 11(b), we observed that the average level for the time-dependent controls $u_{L}(t)$ and $u_{V}(t)$ is close to the upper bound for all temperature values in West Africa. The profile for control $u_{H}(t)$ is low at low temperatures and is high at high temperatures.

This is not the case with infected mosquitoes. Strategy A led to fewer infected mosquitoes compare to Strategy B (see the profile for infected mosquitoes in Figure 17). These strategies perform relatively poorer than when the controls $u_{L}(t), u_{V}(t)$, and $u_{H}(t)$ are all used.

Thus, personal protection against mosquito bite reduces malaria burden in humans better than mosquito-reduction strategies. Mosquito-reduction strategies, on the contrary, led to fewer infected mosquitoes.

\subsubsection{Monocontrol Strategies for Resistance Mosquitoes:} Infected Human Profiles. We compare control Strategies A and $\mathrm{B}$ when insecticide-resistant mosquitoes are present. As with the insecticide-sensitive case, the application of the controls, regardless of the strategy, can greatly reduce the total number of infected humans which is high in the absence of controls (Figures 14 and 18). The average level of all the controls $u_{L}(t), u_{V}(t)$, and $u_{H}(t)$ was used as in the previous sections. Strategy B which involves using only control $u_{H}(t)$ while setting controls $u_{L}(t)=u_{V}(t)=0$ performs better in reducing the number of infected human than Strategy A (which involves using controls $u_{L}(t)$ and $u_{V}(t)$, while setting $\left.u_{H}(t)=0\right)$.

The profile for infected mosquitoes is given in Figure 19, and it shows that Strategy A performs better at reducing infected mosquitoes in the presence of insecticide-resistant mosquitoes.

Thus, personal protection against mosquito bite only as control strategy reduced malaria burden in humans better than mosquito-reduction strategies. However, mosquito-reduction strategies only led to fewer infected mosquitoes when mosquitoes have evolved resistance against insecticide.

\section{Discussion}

Since the 1950s, global temperature has been on the increase with great consequence for vector-borne diseases. It is expected that this warming leads to an expansion in the 


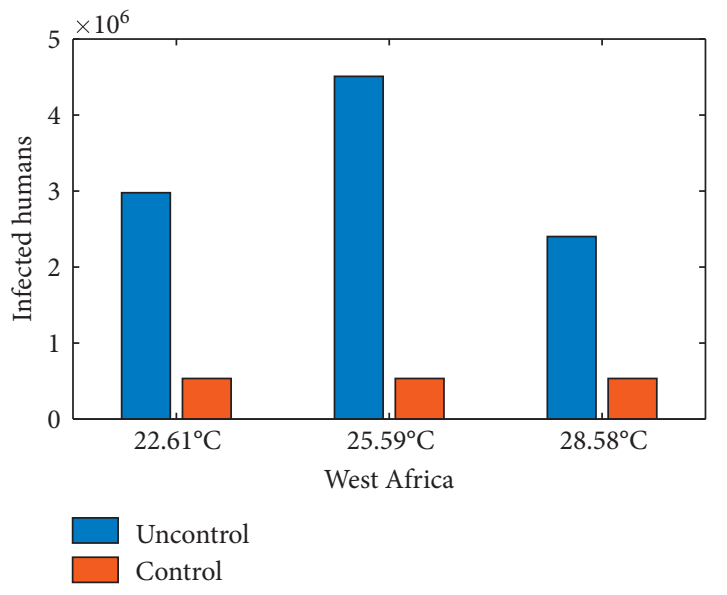

(a)

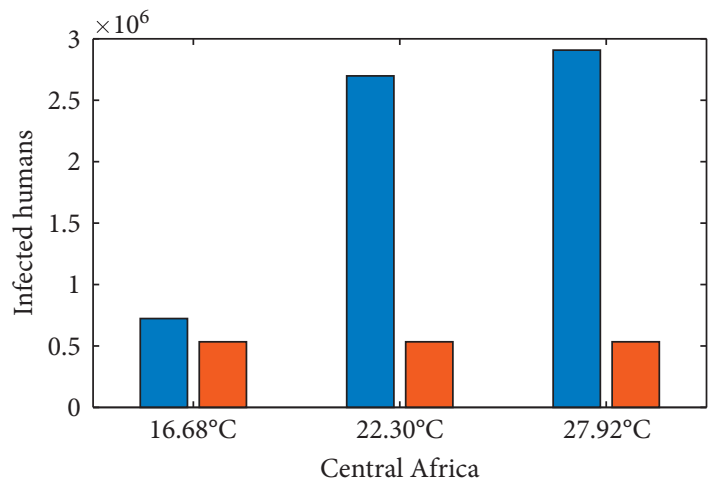

Uncontrol

Control

(c)

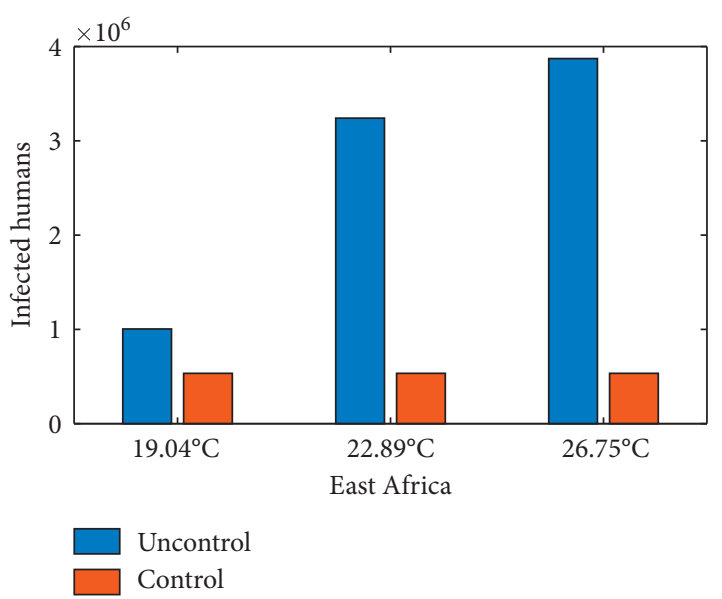

(e)
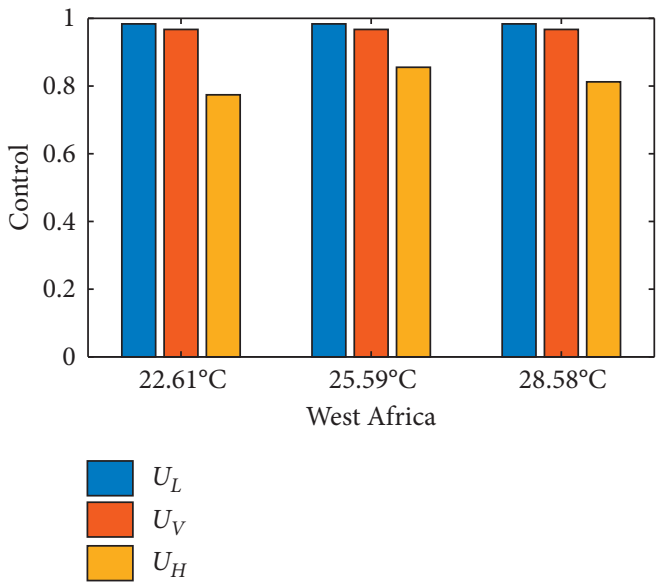

(b)

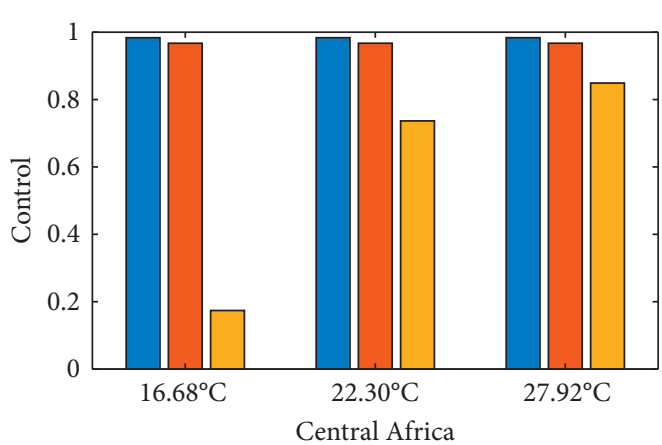

(d)

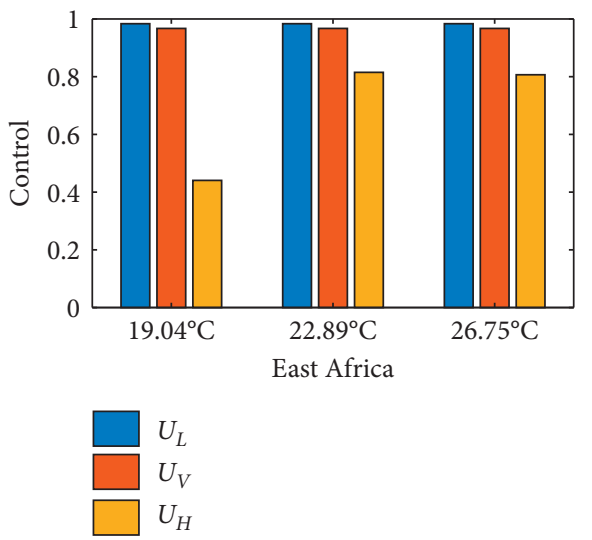

(f)

Figure 14: Continued. 


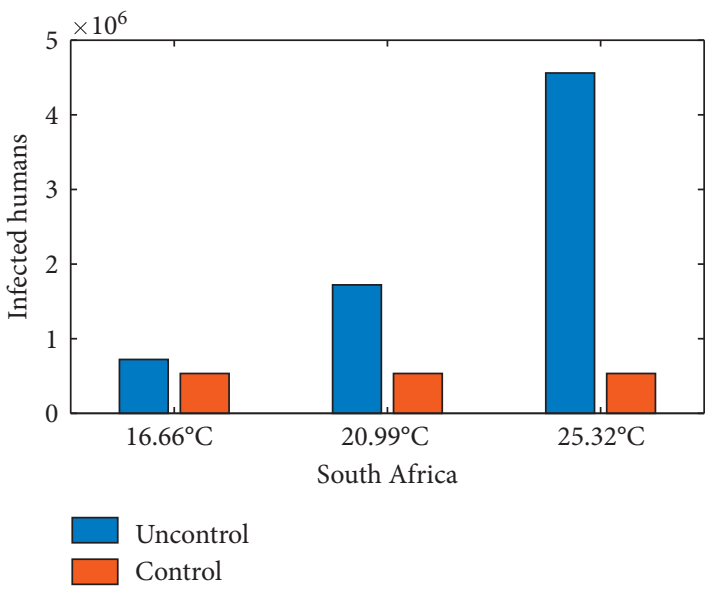

(g)

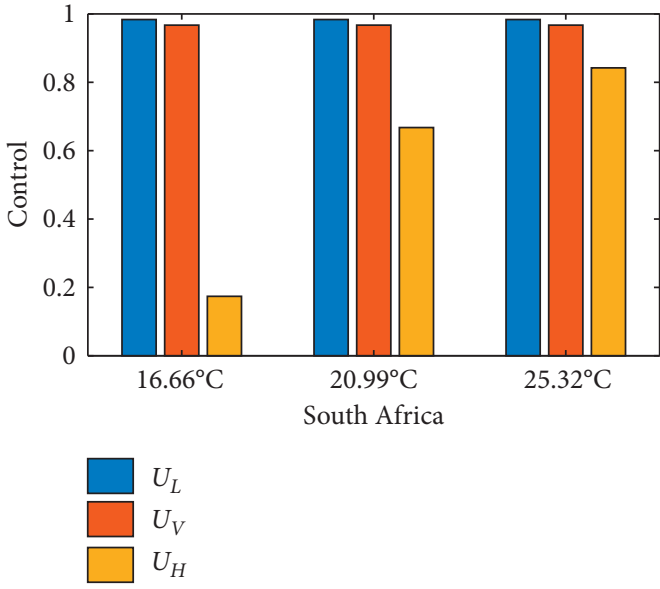

(h)

FIGURE 14: Simulations of the malaria-resistant model (21) as a function of time: (a, c, e, g) the monthly total number of infected humans with control and $(\mathrm{b}, \mathrm{d}, \mathrm{f}, \mathrm{h})$ optimal controls $u_{H}(t), u_{L}(t)$, and $u_{V}(t)$, in West, Central, East, and South Africa.

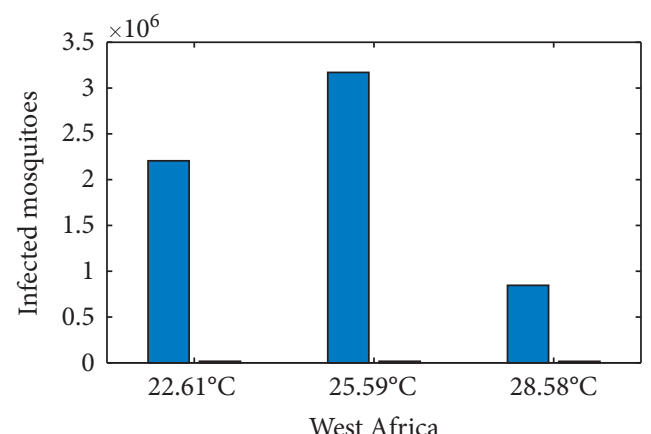

Uncontrol

Control

(a)

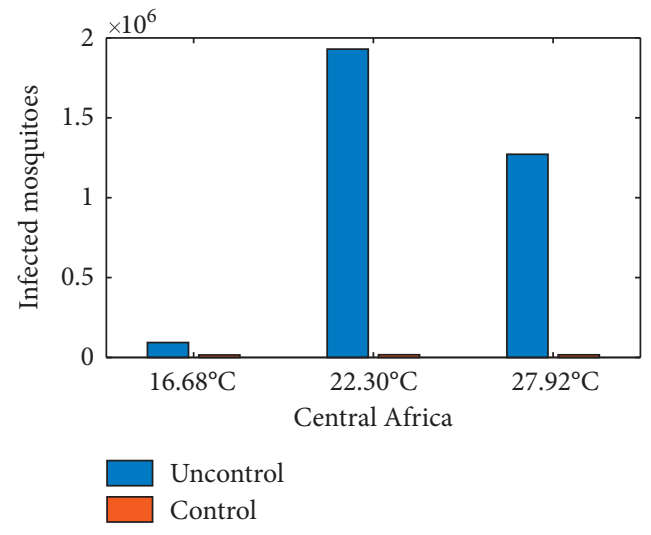

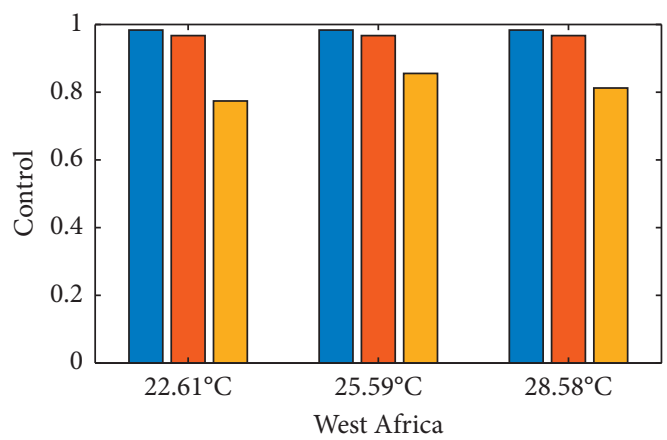
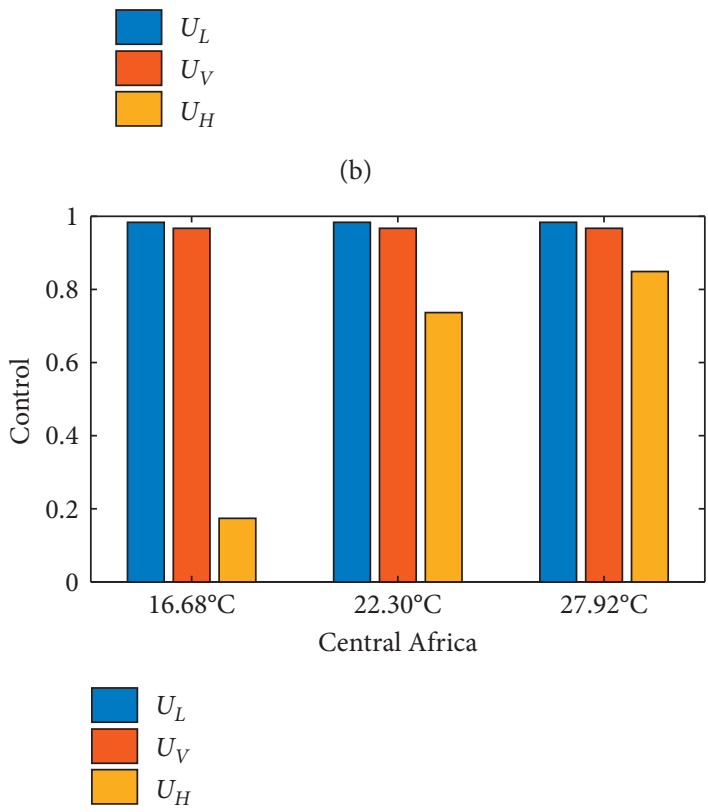

(c) 


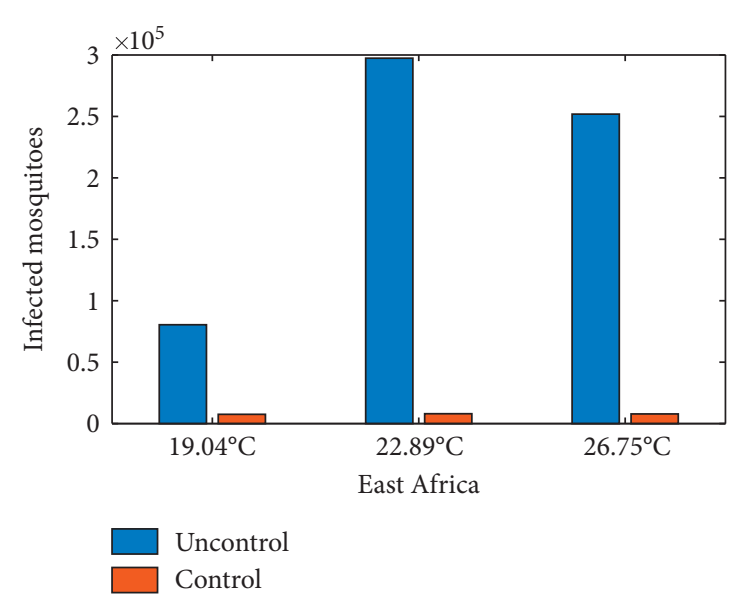

(e)

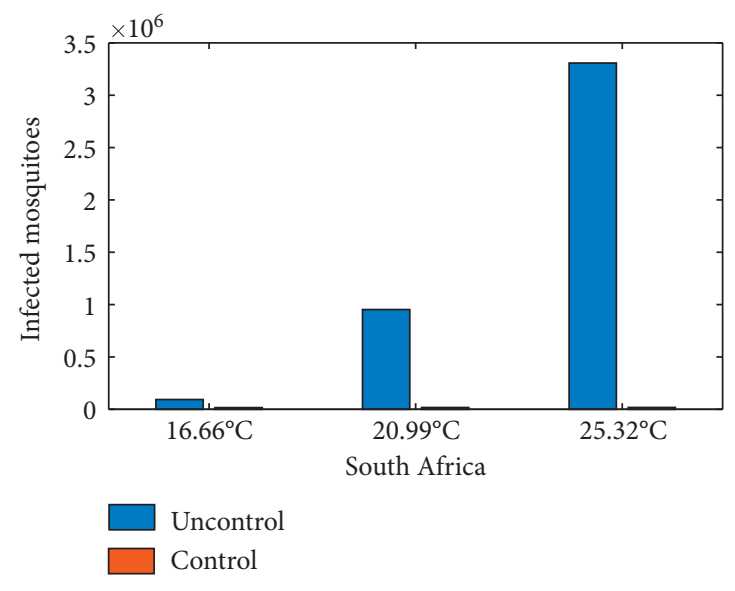

(g)
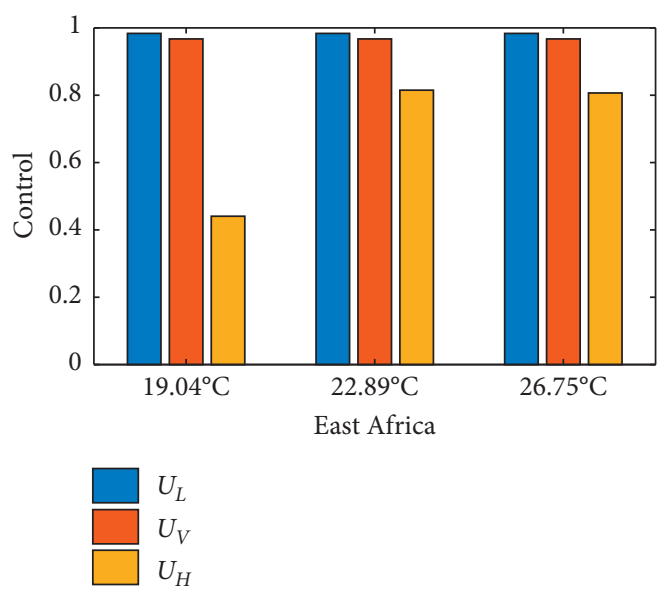

(f)

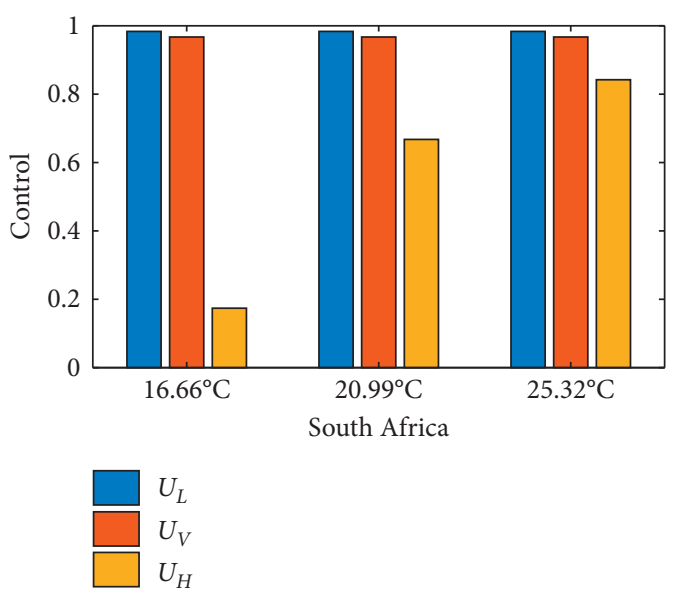

(h)

Figure 15: Simulations of the malaria-resistant model (21) as a function of time in the suitable temperature ranges. (a, c, e) The monthly total number of infected mosquitoes with control and (b, d, f) optimal controls $u_{H}(t), u_{L}(t)$, and $u_{V}(t)$, in West, Central, East, Central, and South Africa.

distribution of vectors into new regions. Several naive populations such as those in cities in the Americas and the Caribbeans have witnessed in recent times increase in emergence and re-emergence of vector-borne diseases such as Chikungunya, Dengue, Yellow Fever, and Zika. Results from Agusto et al. [2] indicate that malaria burden will decrease as temperature goes beyond the range suitable for mosquitoes. According to the World Health Organization (WHO), the global burden of malaria has been on the decline in the last ten years largely due to the use of insecticidetreated bednets [61].

The global cases of malaria increased from 237 million cases in 2010 to 216 million cases in 2016, an 18\% global reduction in incidence rate [61]. This progress has, however, stalled; between 2014 and 2016, there was a substantial increase in malaria incidence cases in the Americas. For instance, in 2015, 211 million cases were reported. This increase in reported cases may be perhaps due to mosquitoes developing resistance to the insecticide used in the bednets [61], particularly pyrethroid [67].

The results from Agusto et al. [2] show that when the mean monthly temperatures are in the range $[16.7,25]^{\circ} \mathrm{C}$, individuals in the community should use both mosquito-reduction strategy and personal protection against mosquito bites; however, when temperatures lie within the range $[26,34]^{\circ} \mathrm{C}$, mosquito-reduction strategy should be used in the community (or household) over personal protection against mosquito bites. In this study, we applied optimal control theory to investigate the optimal control strategy necessary to control the disease in these temperature ranges. We found that in both temperature ranges, the use of mosquito-reduction strategy such as larvicides and adulticides on average should be maintained at relatively high levels which could lead invariably to the development of insecticide-resistant mosquitoes [59, 62].

This result is in line with Barnes et al.'s [59] findings which identified a gene region within Anopheles funestus that allows 


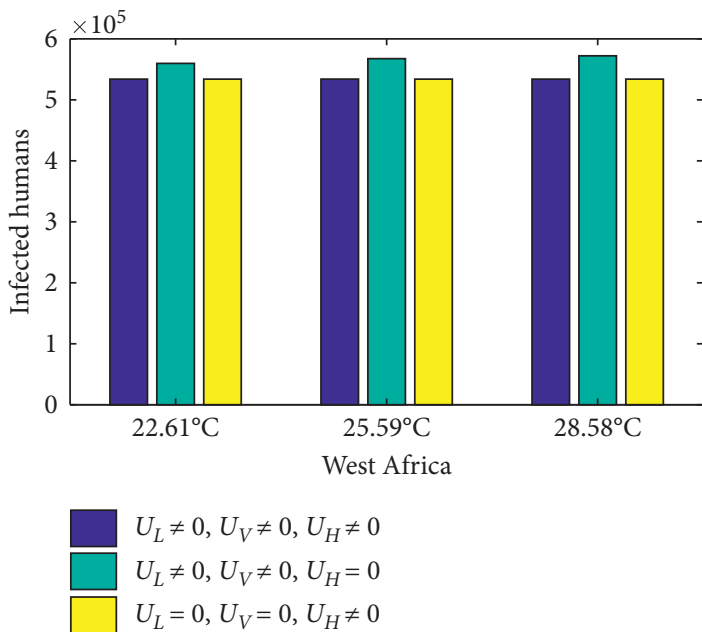

(a)

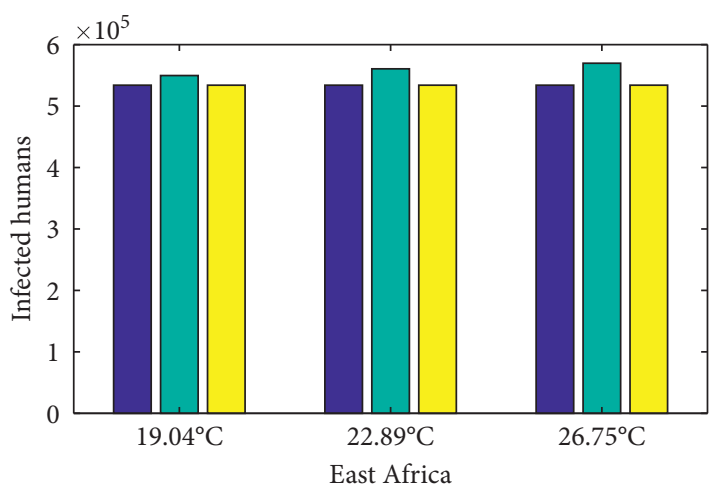

$U_{L} \neq 0, U_{V} \neq 0, U_{H} \neq 0$
$U_{L} \neq 0, U_{V} \neq 0, U_{H}=0$
$U_{L}=0, U_{V}=0, U_{H} \neq 0$

(c)

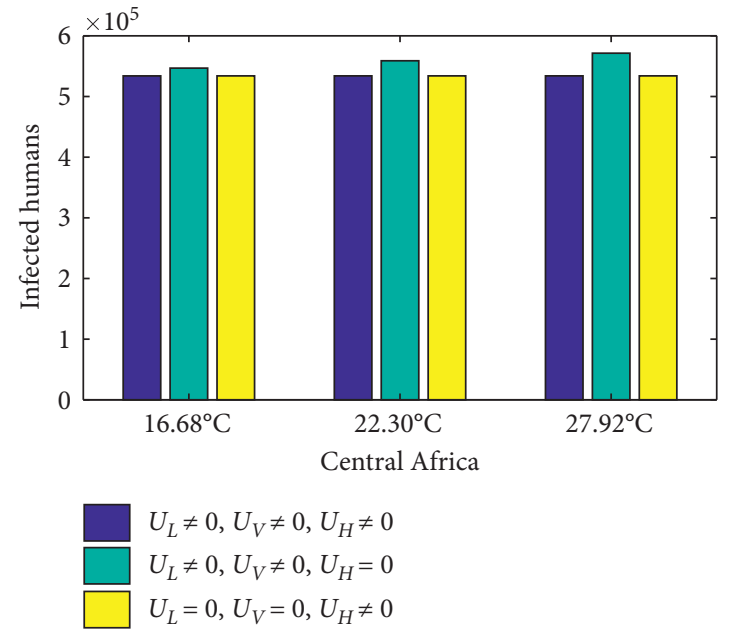

(b)

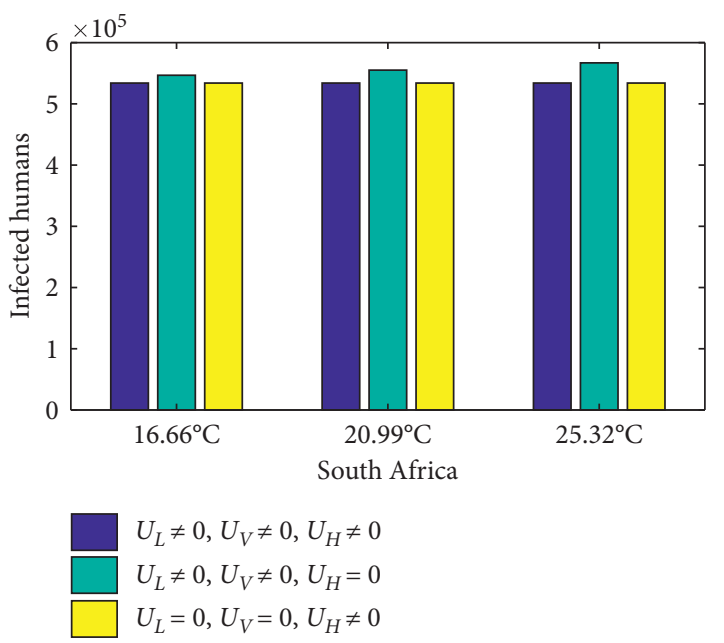

(d)

FIGURE 16: Simulations of the malaria model (2) as a function of time plotting the average control levels for controls $u_{L}, u_{V}$, and $u_{H}$ in West, Central, East, and Central Africa.

the mosquitoes to break down insecticides, thereby resulting in the development of insecticide-resistant mosquitoes. Barnes et al.'s study was a continent-wide population structure of Anopheles funestus mosquitoes. This resistance gene has swept through the entire mosquito populations in southern Africa [60]. According to the WHO, between 2010 and 2016, the frequency of pyrethroid resistance in malaria vectors increased globally [62]. However, the actual impact of insecticide resistance on the effectiveness of insecticidal vector control is not fully known; the development of insecticide resistance highlights a potential challenge to control and elimination of malaria, particularly in Africa where the burden of malaria remains highest [62]. It can undercut the over two decade gain made from malaria control leading to increase in malaria incidence and mortality [68].

The result of this study further shows that control measure aimed at only controlling mosquito population (mosquito-reduction strategies) in the temperature regions suitable for mosquitoes will lead to fewer infected mosquitoes. To effectively control the disease burden, the control measure should prevent or reduce the number of bites humans receive from mosquitoes since personal protection against mosquito bite is better at reducing malaria burden in humans than mosquito-reduction strategies.

When mosquitoes have evolved resistance against insecticide, personal protection against mosquito is equally better than mosquito-reduction strategies (larvacides and adulticides) in reducing malaria burden in humans. However, mosquito-reduction strategies only led to fewer infected mosquitoes. To effectively control the mosquito population, given the added pressure of insecticide resistance, a higher level of larvicides and adulticides is needed. This level is higher than the level used when mosquitoes are sensitive to insecticides. We also observed (not shown here) that as the ratio $(f)$ of sensitive to resistance mosquitoes increases in the resistance direction, the level of optimal control of larvacides and adulticides remained constant and close to $100 \%$. 

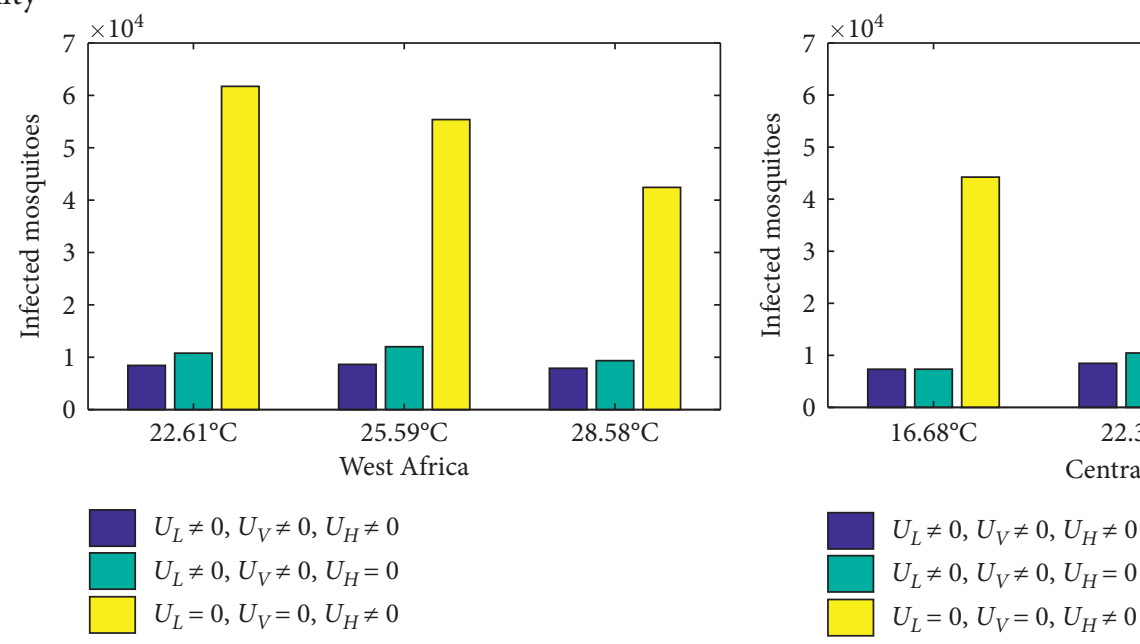

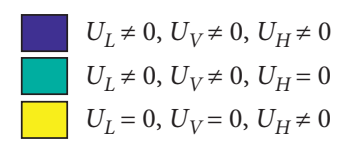

(a)

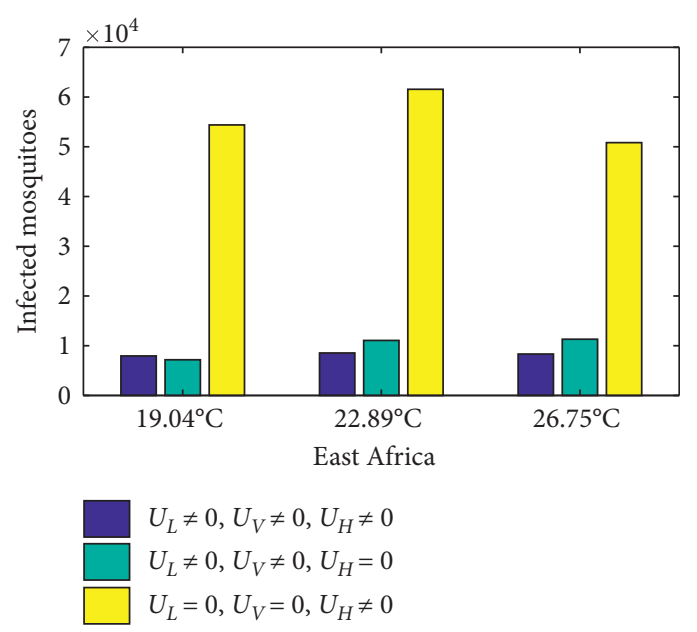

(c)

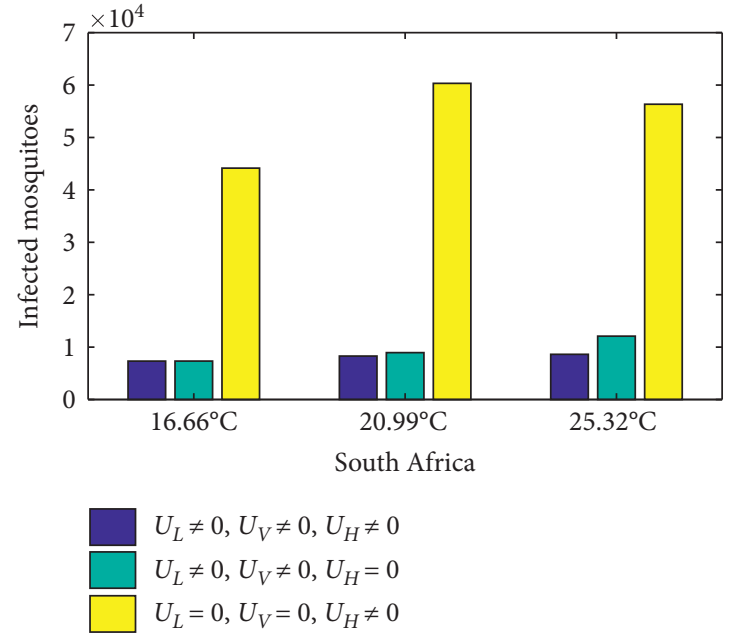

(d)

Figure 17: Simulations of the malaria model (2) as a function of time plotting the average control levels for controls $u_{L}, u_{V}$, and $u_{H}$ in West, Central, East, and Central Africa.

As observed in this study of insecticide-resistant mosquitoes, control and elimination of malaria in the presence of resistance mosquitoes are possible only with the use of high level of insecticide use. This then leads to a vital question: "will the evolutionary adaptation to insecticides then lead to supermosquitoes developing resistance to all insecticide as observed with bacteria with antimicrobial resistance?"

Therefore, to prevent such catastrophic development, efforts should be geared towards exploring other efficient ways and strategies to control malaria and prevent the development of insecticide-resistant mosquitoes by targeting either the mosquito larvae or adult stages.

Efforts aimed at malaria control can help reduce the development of insecticide mosquitoes; for instance, active participation of the community and government through community-based interventions is essential for community wide disease elimination and control using strategies that interrupt disease transmission such as backyard draining of standing water [69], improve drainage, and community education regarding introducing a novel vector control strategy like the genetically engineered insects [70-73].

Other useful strategies that can reduce the development of insecticide-resistant mosquitoes target the larvae stage through the use of larvicides such as larviciding with larvivorous fish and bacterial larvicide [40-43, 45-48].

Larvivorous fish have been employed as biological control of mosquito larvae since 1937 [74-79]. They target mosquito larvae and are safe for humans since they are devoid of insecticide [44]. Larvivorous fish are well suited for urban areas where the density of humans needing protection is higher than mosquito breeding sites [76] and places with seasonal transmission [44].

Bacterial larvicides is another mosquito larvae targeting the control method using Bacillus thuringiensis var. israelensis (Bti) and Bacillus sphaericus (Bs) [45-48]. These microbial larvacides selectively kills the mosquito larvae when they ingest the bacterial spores leaving unharmed other cohabiting organisms [80].

Thus, implementing these larvae control strategies in addition to the use of bednets and adult control will not only 

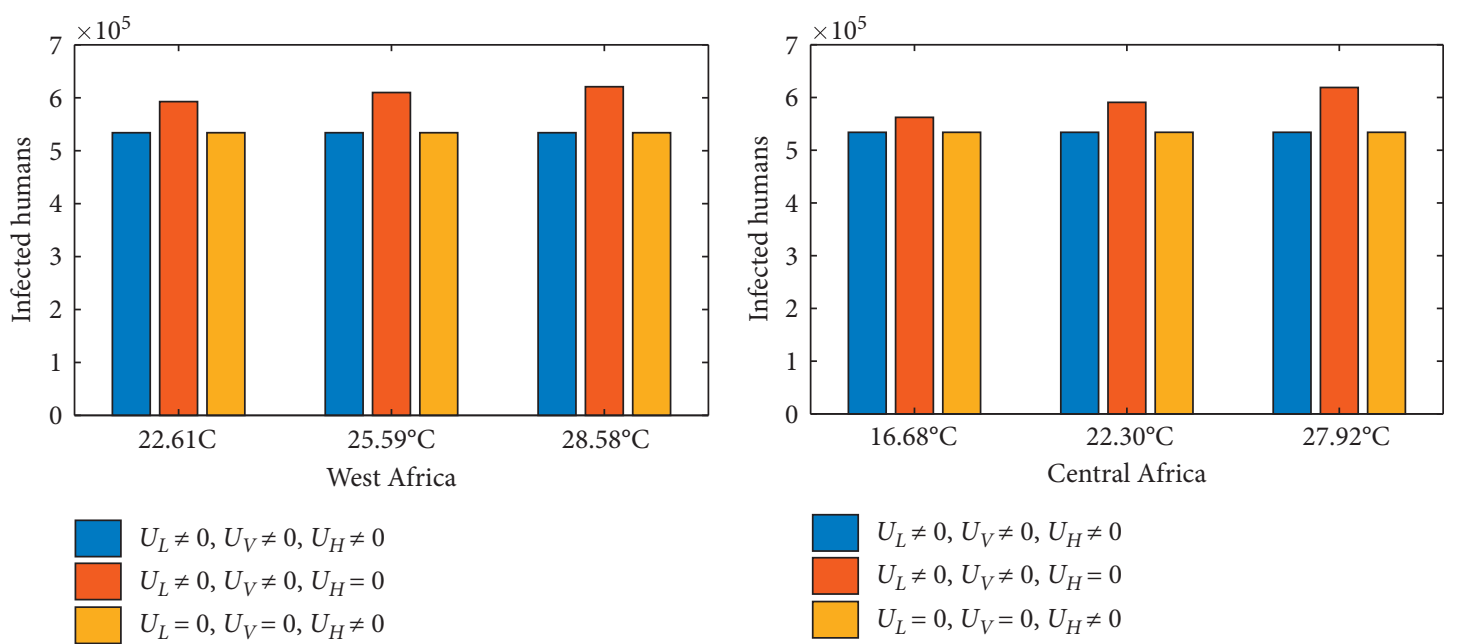

(a)

(b)

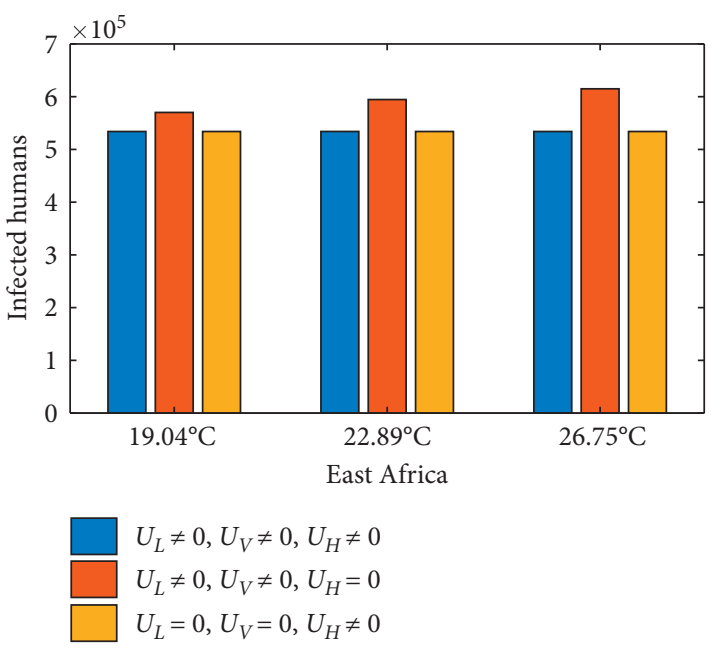

(c)

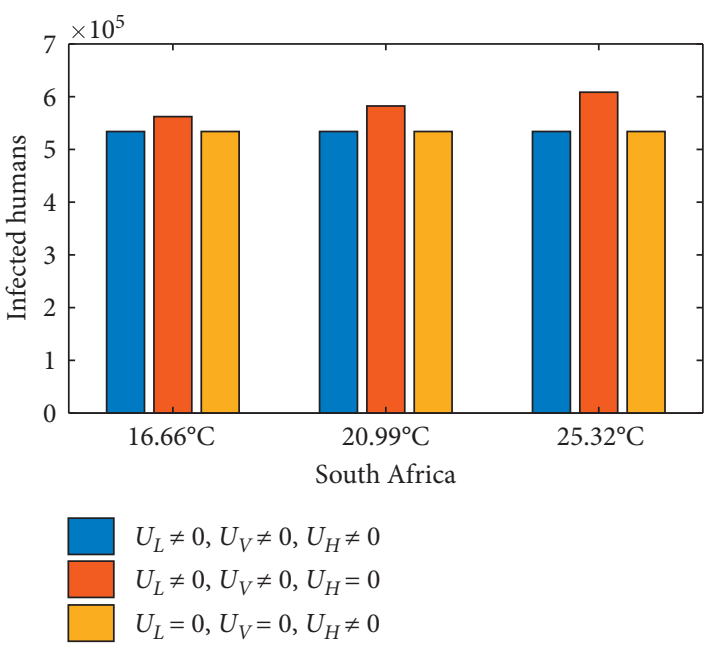

(d)

FIGURE 18: Simulations of the malaria-resistant model (21) as a function of time plotting the average control levels for controls $u_{L}, u_{V}$, and $u_{H}$ in West, Central, East, Central, and South Africa.

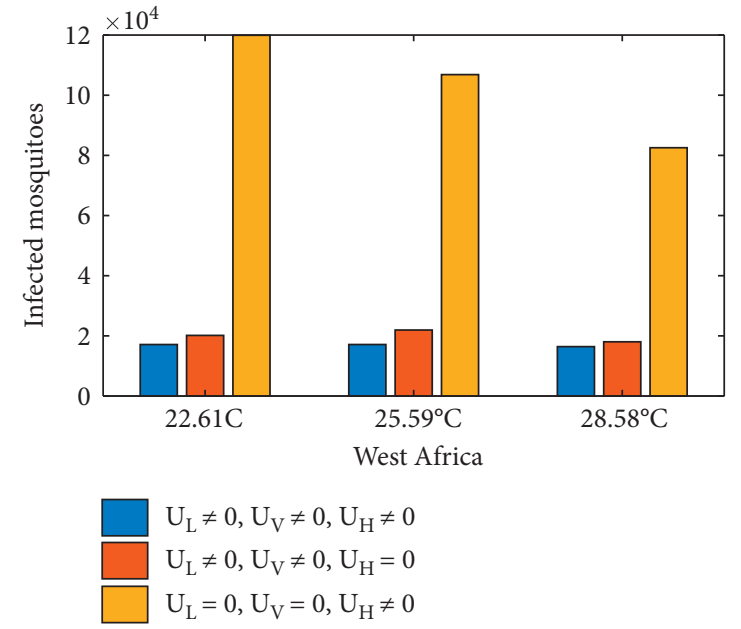

(a)

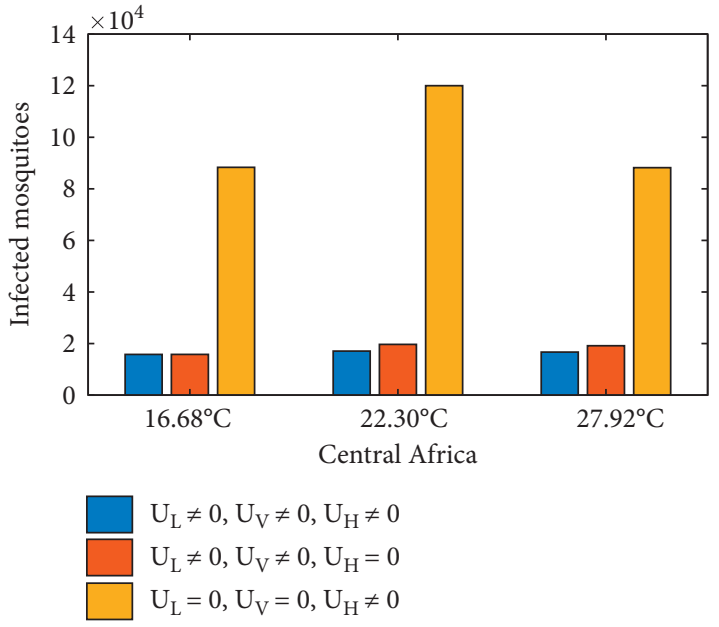

(b)

Figure 19: Continued. 


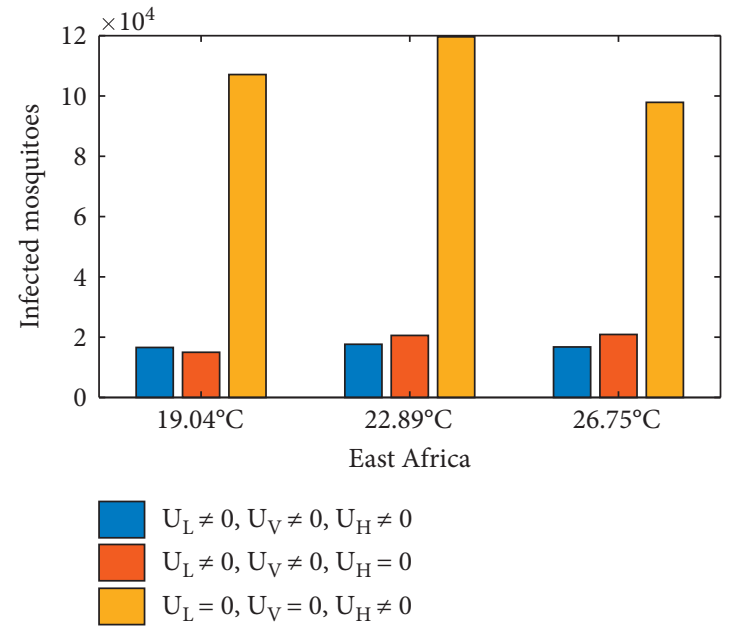

(c)

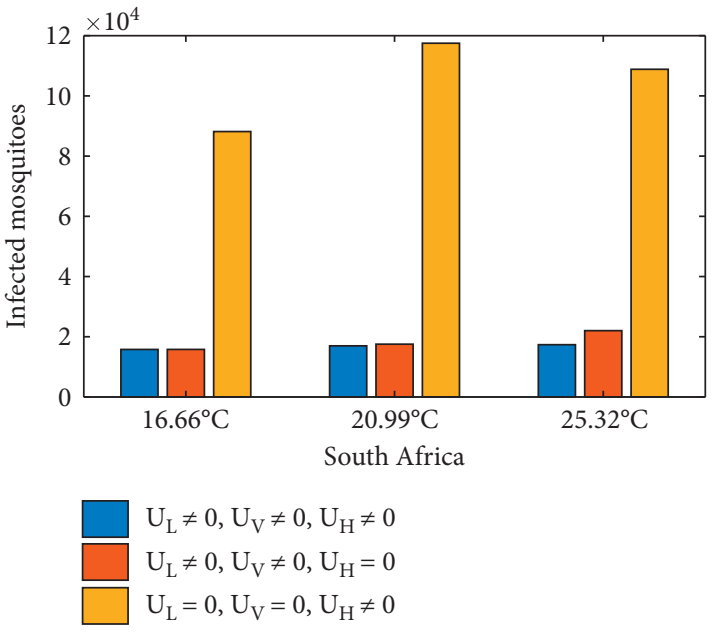

(d)

Figure 19: Simulations of the malaria-resistant model (21) as a function of time plotting the average control levels for controls $u_{L}$, $u_{V}$, and $u_{H}$ in West, Central, East, Central, and South Africa.

reduce the chance of developing insecticide-resistant mosquitoes, it will ultimately lead to control and elimination of malaria in a community.

\section{Conclusions}

In this study, we applied optimal control theory to the model developed in Agusto et al. [2] in order to determine if individuals in the community should use both mosquito-reduction strategy and personal protection against mosquito bites for mean monthly temperatures are in the range $[16.7,25]^{\circ} \mathrm{C}$ or they should emphasize mosquito-reduction strategy in the community (or household) ahead of personal protection for higher mean monthly temperatures in the range $[26,34]^{\circ} \mathrm{C}$.

Using the simple model and results in [2], we summarized below some of the findings of this study. Detailed theoretical and epidemiological findings of the model are given in [2]:

(i) Numerical simulations of the model (2) show that the total number of new cases of infection (malariaassociated burden) increases with increasing mean monthly temperature in the following ranges:

(a) $\left[22.61^{\circ} \mathrm{C}, 28.58^{\circ} \mathrm{C}\right]$ in the three West African cities.

(b) $\left[16.68^{\circ} \mathrm{C}, 27.92^{\circ} \mathrm{C}\right]$ in the three Central African cities.

(c) $\left[19.04^{\circ} \mathrm{C}, 26.75^{\circ} \mathrm{C}\right]$ in the three East African cities.

(d) $\left[16^{\circ} \mathrm{C}, 25^{\circ} \mathrm{C}\right]$ in KwaZulu-Natal, South Africa.

(ii) Malaria burden in the presence of temperature variations can be decreased via the application of time-dependent controls.

(iii) The solution profile of the personal protection control mimics the response of infected mosquitoes to temperature in the absence of control. (iv) Malaria burden can be decreased in the presences of insecticide-resistant and temperature variations.

(v) With monocontrol strategies, personal protection against mosquito bite is better at reducing malaria burden in humans than mosquito-reduction strategies, and mosquito-reduction strategies led to fewer infected mosquitoes.

Thus, we conclude that personal protection, particularly the use of bednets, should be encouraged not only at low temperatures but particularly at high temperatures when individuals are driven not to use the nets. Furthermore, control and elimination of malaria are possible even with mosquitoes developing resistance to the insecticide. In future work, we will incorporate the kinetics of insecticide-treated bednets, larvacides (with either larvivorous fish or bacterial larvicide), and adulticides (which will decay in some functional form). We will also incorporate rainfall and seasonality to address the possibility of control and resistance prevention using optimal control in areas with seasonal malaria.

\section{Appendix}

\section{A. Characterization of Optimal Controls}

We seek to find the optimal controls $u_{H}^{*}, u_{L}^{*}$, and $u_{V}^{*}$ such that

$$
J\left(u_{H}^{*}, u_{L}^{*}, u_{V}^{*}\right)=\min _{\mathcal{U}}\left\{J\left(u_{H}, u_{L}, u_{V}\right)\right\},
$$

where the control set

$$
\mathcal{U}=\left\{\left(u_{H}, u_{L}, u_{V}\right):\left[0, t_{f}\right] \longrightarrow[0,1], \text { is Lebesgue measurable }\right\} .
$$

The controls $\left(u_{H}(t), u_{L}(t), u_{V}(t)\right)$ are bounded Lebesgue integrable functions [50-54].

The optimal control triple $\left(u_{H}(t), u_{L}(t)\right.$, and $\left.u_{V}(t)\right)$ satisfy necessary conditions from Pontryagin's maximum 
principle [81]. This principle converts (15) and (16) into a problem of minimizing pointwise a Hamiltonian $H$, with respect to the controls $\left(u_{H}(t), u_{L}(t)\right.$, and $\left.u_{V}(t)\right)$. The optimality conditions are obtained using the Hamiltonian formulated from the cost functional (16) and the governing dynamics (15). Thus, Hamiltonian $(H)$ is given as

$$
\begin{aligned}
H= & A_{1} I_{H}+B_{1} L_{V}+B_{2} S_{V}+B_{3} E_{V}+B_{4} I_{V} \\
& +C_{1} u_{H}+\varepsilon C_{2} u_{H}^{2}+C_{3} u_{L}+\varepsilon C_{4} u_{L}^{2}+C_{5} u_{V}+\varepsilon C_{6} u_{V}^{2} \\
& +\lambda_{S_{H}}\left[\Pi_{H}+\omega_{1} R_{H}(t)-\lambda_{H}\left[T,\left(1-u_{H}(t)\right) I_{V}, N_{H}\right] S_{H}(t)-\mu_{H} S_{H}(t)\right] \\
& +\lambda_{E_{H}}\left\{\lambda_{H}\left[T,\left(1-u_{H}(t)\right) I_{V}, N_{H}\right] S_{H}(t)-\left(\sigma_{H}+\mu_{H}\right) E_{H}(t)\right\} \\
& +\lambda_{I_{H}}\left\{\sigma_{1} E_{H}(t)-\left(\gamma_{1}+\mu_{H}+\delta_{H}\right) I_{H}(t)\right\} \\
& +\lambda_{R_{H}}\left[\gamma_{1} I_{H}(t)-\left(\omega_{1}+\mu_{H}\right) R_{H}(t)\right] \\
& +\lambda_{L_{V}}\left[\phi_{V}(T)\left(1-\frac{L_{V}(t)}{K_{V}}\right)\left[S_{V}(t)+E_{V}(t)+I_{V}(t)\right]-\left[\theta_{V}(\widehat{T})+\mu_{L}(\widehat{T})\right] L_{V}(t)-u_{L}(t) L_{V}(t)\right] \\
& +\lambda_{S_{V}}\left[\theta_{V}(\widehat{T}) L_{V}(t)-\lambda_{V}\left[T,\left(1-u_{H}(t)\right) N_{H}\right] S_{V}(t)-\mu_{V}(T) S_{V}(t)-u_{V}(t) S_{V}(t)\right] \\
& +\lambda_{E_{V}}\left[\lambda_{V}\left[T,\left(1-u_{H}(t)\right) N_{H}\right] S_{V}(t)-\left[\sigma_{V}(T)+\mu_{V}(T)\right] E_{V}(t)-u_{V}(t) E_{V}(t)\right] \\
& +\lambda_{I_{V}}\left[\sigma_{V}(T) E_{V}(t)-\mu_{V}(T) I_{V}(t)-u_{V}(t) I_{V}(t)\right],
\end{aligned}
$$

where $\lambda_{S_{H}}, \lambda_{E_{H}}, \lambda_{I_{H}}, \lambda_{R_{H}}, \lambda_{L_{V}}, \lambda_{S_{V}}, \lambda_{E_{V}}$, and $\lambda_{I_{V}}$ are the associated adjoints for the state variables $S_{H}, E_{H}, I_{H}, R_{H}, L_{V}, S_{V}, E_{V}$, and $I_{V}$. The adjoint equation system is determined from the partial derivatives of the Hamiltonian (A.3) with respect to the state and control variables.

Theorem 1. Given the optimal controls $\left(u_{H}^{*}, u_{L}^{*}, u_{V}^{*}\right)$ and the solutions of the corresponding state system (15) $\left(S_{H}^{*}, E_{H}^{*}, I_{H}^{*}, R_{H}^{*}, L_{V}^{*}, S_{V}^{*}, E_{V}^{*}, I_{V}^{*}\right)$ which minimizes the objective functional $J\left(u_{H}^{*}, u_{L}^{*}, u_{V}^{*}\right)$ over $\mathcal{U}$. Then, there exist adjoint variables $\lambda_{S_{H}}, \lambda_{E_{H}}, \lambda_{I_{H}}, \lambda_{R_{H}}, \lambda_{L_{V}}, \lambda_{S_{V}}, \lambda_{E_{V}}, \lambda_{I_{V}}$, satisfying the system

$$
-\frac{d \lambda_{i}}{\mathrm{~d} t}=\frac{\partial H}{\partial i}
$$

and with transversality conditions

$\lambda_{i}\left(t_{f}\right)=0$, where $i=S_{H}, E_{H}, I_{H}, R_{H}, L_{V}, S_{V}, E_{V}, I_{V}$.

The optimality conditions is given as

$$
\frac{\partial H}{\partial u_{j}}=0, \quad j=H, L, V .
$$

Furthermore, the control $\left(u_{H}^{*}, u_{L}^{*}, u_{V}^{*}\right)$ is given as

$$
\begin{aligned}
& u_{H}^{*}=\min \left\{1, \max \left[0, \frac{1}{2 \varepsilon C_{2} N_{H}^{*}}\left(-C_{1} N_{H}^{*}+S_{V}^{*} b_{V}(T) \beta_{V}\left(\lambda_{E_{V}}^{*}-\lambda_{S_{V}}^{*}\right) I_{H}^{*}+I_{V}^{*} b_{V}(T) \beta_{H}\left[\left(\lambda_{E_{H}}^{*}-\lambda_{S_{H}}^{*}\right) S_{H}^{*}\right]\right)\right]\right\}, \\
& u_{L}^{*}=\min \left\{1, \max \left[0, \frac{\lambda_{L_{V}}^{*} L_{V}^{*}-C_{3}}{2 \varepsilon C_{4}}\right]\right\}, \\
& u_{V}^{*}=\min \left\{1, \max \left[0, \frac{\lambda_{E_{V}}^{*} E_{V}+\lambda_{I_{V}}^{*} I_{V}^{*}+\lambda_{S_{V}}^{*} S_{V}^{*}-C_{5}}{2 \varepsilon C_{6}}\right]\right\} .
\end{aligned}
$$

Proof. Using the result by Fleming and Rishel [82], the existance of the optimal control can be shown. Thus, taking the partial derivative of the Hamiltonian function $(H)$ with respect to the state variables leads to the system of adjoint equations. Thus, the adjoint system is given as 


$$
\begin{array}{cc}
-\frac{d \lambda_{S_{H}}}{\mathrm{~d} t}=\frac{\partial H}{\partial S_{H}}, & \lambda_{S_{H}}\left(t_{f}\right)=0, \\
\cdots & \\
-\frac{d \lambda_{I_{H}}}{\mathrm{~d} t}=\frac{\partial H}{\partial I_{H}}, & \lambda_{I_{H}}\left(t_{f}\right)=0, \\
-\frac{d \lambda_{R_{H}}}{\mathrm{~d} t}=\frac{\partial H}{\partial R_{H}}, & \lambda_{R_{H}}\left(t_{f}\right)=0, \\
-\frac{d \lambda_{L_{V}}}{\mathrm{~d} t}=\frac{\partial H}{\partial L_{V}}, & \lambda_{L_{V}}\left(t_{f}\right)=0, \\
\cdots & \\
-\frac{d \lambda_{I_{V}}}{\mathrm{~d} t}=\frac{\partial H}{\partial I_{V}}, & \lambda_{I_{V}}\left(t_{f}\right)=0,
\end{array}
$$

evaluating the adjoint system at the optimal controls and corresponding state variables, the stated adjoint systems (A.4) and (A.5) are given. In addition, the controls $\left(u_{H}^{*}, u_{L}^{*}, u_{V}^{*}\right)$ are obtained by taking the partial derivative of the Hamiltonian function, $H$, with respect to the control variables in the interior of the control set and then solving for controls $\left(u_{H}^{*}, u_{L}^{*}, u_{V}^{*}\right)$; hence, we have the result for the optimality conditions given as

$$
\begin{aligned}
\frac{\partial H}{\partial u_{H}}= & 2 \varepsilon C_{2} u_{H}^{*}-\left[-C_{1} N_{H}^{*}+S_{V}^{*} b_{V}(T) \beta_{V}\left(\lambda_{E_{V}}^{*}-\lambda_{S_{V}}^{*}\right) I_{H}^{*}\right. \\
& \left.+I_{V}^{*} b_{V}(T) \beta_{H}\left[\left(\lambda_{E_{H}}^{*}-\lambda_{S_{H}}^{*}\right) S_{H}^{*}\right]\right]=0, \\
\frac{\partial H}{\partial u_{L}}= & 2 \varepsilon C_{4} u_{L}^{*}-\left[\lambda_{L_{V}}^{*} L_{V}^{*}-C_{3}\right]=0, \\
\frac{\partial H}{\partial u_{V}}= & 2 \varepsilon C_{6} u_{V}^{*}-\left[\lambda_{E_{V}}^{*} E_{V}+\lambda_{I_{V}}^{*} I_{V}^{*}+\lambda_{S_{V}}^{*} S_{V}^{*}-C_{5}\right]=0 .
\end{aligned}
$$

Solving for $u_{H}^{*}, u_{V}^{*}$, and $u_{M}^{*}$, we have

$$
\begin{aligned}
u_{H}^{*} & =\frac{1}{2 \varepsilon C_{2}}\left(-C_{1} N_{H}^{*}+S_{V}^{*} b_{V}(T) \beta_{V}\left(\lambda_{E_{V}}^{*}-\lambda_{S_{V}}^{*}\right) I_{H}^{*}\right. \\
& \left.+I_{V}^{*} b_{V}(T) \beta_{H}\left[\left(\lambda_{E_{H}}^{*}-\lambda_{S_{H}}^{*}\right) S_{H}^{*}-\lambda_{R_{H}}^{*} R_{H}^{*}\right]\right), \\
u_{L}^{*}= & \frac{\lambda_{L_{V}}^{*} L_{V}^{*}-C_{3}}{2 \varepsilon C_{4}}, \\
u_{V}^{*}= & \frac{\lambda_{E_{V}}^{*} E_{V}+\lambda_{I_{V}}^{*} I_{V}^{*}+\lambda_{S_{V}}^{*} S_{V}^{*}-C_{5}}{2 \varepsilon C_{6}} .
\end{aligned}
$$

The characterization (A.7) can be derived by using the bounds on the controls.

Remark 1. The uniqueness of the optimal control for a small time $\left(t_{f}\right)$ can be shown using the a priori boundedness of the state and adjoint functions along with the resulting Lipschitz structure of the ODE's. Therefore, the uniqueness of the optimal control follows from the uniqueness of the optimality system, which consists of (15) and (A.4), (A.5) with characterization (A.7). The restriction on the length of the time to a small interval is to guarantee the uniqueness of the optimality system. The small length of time is due to the opposite time orientations of (15), (A.4), and (A.5); the state problem has initial values, and the adjoint problem has final values. This restriction is very common in optimal control problems $[51,53,54]$.

\section{Data Availability}

The temperature data are within the plots. And they can be made available upon request.

\section{Conflicts of Interest}

The author declares no conflicts of interest.

\section{Acknowledgments}

This research was supported by the Strategic Environmental Research and Development Program under grant RC-2639 and University of Kansas General Research Grant 23012105075 .

\section{References}

[1] World Health Organization, Malaria, WHO, Geneva, Switzerland, 2010, http://www.who.int/mediacentre/factsheets/ fs094/en/.

[2] F. B. Agusto, A. B. Gumel, and P. E. Parham, "Qualitative assessment of the role of temperature variations on malaria transmission dynamics," Journal of Biological Systems, vol. 23, no. 4, Article ID 1550030, 2015.

[3] J. Hansen, M. Sato, and R. Ruedy, "Perception of climate change," Proceedings of the National Academy of Sciences, vol. 109, no. 37, pp. E2415-E2423, 2012.

[4] R. S. Ostfeld, "Climate change and the distribution and intensity of infectious diseases," Ecology, vol. 90, no. 4, pp. 903-905, 2009.

[5] P. Reiter, "Climate change and mosquito-borne disease," Environmental Health Perspectives, vol. 109, no. 1, p. 141, 2001.

[6] D. J. Rogers and S. E. Randolph, "The global spread of malaria in a future, warmer world," Science, vol. 289, no. 5485, pp. 1763-1766, 2000.

[7] J. R. Rohr, A. P. Dobson, P. T. J. Johnson et al., "Frontiers in climate change-disease research," Trends in Ecology \& Evolution, vol. 26, no. 6, pp. 270-277, 2011.

[8] Intergovernmental Panel on Climate Change, "Fourth assessment report: climate change 2007: working group i report: the physical science basis," IPCC, Geneva, Switzerland, 2007.

[9] Roll Back Malaria, Climate Change and Malaria, http://www. rollbackmalaria.org/wp-content/uploads/2017/08/RBM Climate_Change_Fact-Sheet_170915.pdf, 2015.

[10] The National Center for Atmospheric Research (NCAR), Climate Change and Vector-Borne Disease.

[11] The United Nations (UN), "Climate change and malaria - a complex relationship," UNChronicle, vol. XLVII, no. 2, 2010.

[12] A. K. Githeko, S. W. Lindsay, U. E. Confalonieri, and J. A. Patz, "Climate change and vector-borne diseases: a regional analysis," Bulletin of the World Health Organization, vol. 78, no. 9, pp. 1136-1147, 2000. 
[13] J. A. Patz and W. K. Reisen, "Immunology, climate change and vector-borne diseases," Trends in Immunology, vol. 22, no. 4, pp. 171-172, 2001.

[14] L. M. Rueda, K. J. Patel, R. C. Axtell, and R. E. Stinner, "Temperature-dependent development and survival rates of culex quinquefasciatus and aedes aegypti (diptera: Culicidae)," Journal of Medical Entomology, vol. 27, no. 5, pp. 892-898, 1990.

[15] A. Egbendewe-Mondzozo, M. Musumba, B. A. McCarl, and $\mathrm{X}$. $\mathrm{Wu}$, "Climate change and vector-borne diseases: an economic impact analysis of malaria in africa," International Journal of Environmental Research and Public Health, vol. 8, no. 3, pp. 913-930, 2011.

[16] M. J. Turell, "Effect of environmental temperature on the vector competence of aedes fowleri for rift valley fever virus," Research in Virology, vol. 140, pp. 147-154, 1989.

[17] O. Faye, O. Gaye, D. Fontenille et al., "Drought and malaria decrease in the niayes area of Senegal," Sante (Montrouge, France), vol. 5, no. 5, pp. 299-305, 1995.

[18] D. K. De Souza, P. N. Owusu, and M. D. Wilson, "Impact of climate change on the geographic scope of diseases," in Human and Social Dimensions of Climate ChangeInTech, London, UK, 2012.

[19] P. E. Parham and E. Michael, "Modeling the effects of weather and climate change on malaria transmission," Environmental Health Perspectives, vol. 118, no. 5, p. 620, 2010.

[20] A. K. Githeko, "Malaria and climate change: special feature," in Commonwealth Health Ministers' Update 2009Commonwealth Secretariat: Pro-Brook Publishing, London, UK, 2009.

[21] D. LeSueur, M. Craig, C. Fraser et al., "Towards an atlas of malaria risk in africa: first technical report of the mara/arma collaboration," 1998, https://idl-bnc-idrc.dspacedirect.org/ bitstream $/$ handle/10625/31644/114833.pdf? sequence $=1$.

[22] K. P. Paaijmans, M. O. Wandago, A. K. Githeko, and W. Takken, "Unexpected high losses of anopheles gambiae larvae due to rainfall," PLoS One, vol. 2, no. 11, Article ID e1146, 2007.

[23] K. Blayneh, Y. Cao, Y. Cao, and H.-D. Kwon, "Optimal control of vector-borne diseases: treatment and prevention," Discrete \& Continuous Dynamical Systems-B, vol. 11, no. 3, pp. 587-611, 2009.

[24] C. Bowman, A. Gumel, P. Vandendriessche, J. Wu, and H. Zhu, "A mathematical model for assessing control strategies against west nile virus," Bulletin of Mathematical Biology, vol. 67, no. 5, pp. 1107-1133, 2005.

[25] A. M. Niger and A. B. Gumel, "Mathematical analysis of the role of repeated exposure on malaria transmission dynamics," Differential Equations and Dynamical Systems, vol. 16, no. 3, pp. 251-287, 2008.

[26] Centers for Disease Control and Prevention(CDC), Anopheles Mosquitoes, http://www.cdc.gov/malaria/about/biology/ mosquitoes/, 2012.

[27] Wikipedia, “Anopheles," 2013, http://en.wikipedia.org/wiki/ Anopheles\\#Lifel_stages.

[28] K. Brugger and F. Rubel, "Simulation of climate-change scenarios to explain usutu-virus dynamics in Austria," Preventive Veterinary Medicine, vol. 88, no. 1, pp. 24-31, 2009.

[29] V. Laperriere, K. Brugger, and F. Rubel, "Simulation of the seasonal cycles of bird, equine and human west nile virus cases," Preventive Veterinary Medicine, vol. 98, no. 2-3, pp. 99-110, 2011.

[30] F. Rubel, K. Brugger, M. Hantel et al., "Explaining usutu virus dynamics in Austria: model development and calibration,"
Preventive Veterinary Medicine, vol. 85, no. 3-4, pp. 166-186, 2008.

[31] M. N. Bayoh and S. W. Lindsay, "Effect of temperature on the development of the aquatic stages of anopheles gambiae sensu stricto (diptera: Culicidae)," Bulletin of Entomological Research, vol. 93, no. 5, pp. 375-381, 2003.

[32] C. J. M. Koenraadt, K. P. Paaijmans, P. Schneider, A. K. Githeko, and W. Takken, "Low larval vector survival explains unstable malaria in the western Kenya highlands," Tropical Medicine and International Health, vol. 11, no. 8, pp. 1195-1205, 2006.

[33] K. P. Paaijmans, A. F. Read, and M. B. Thomas, "Understanding the link between malaria risk and climate," Proceedings of the National Academy of Sciences, vol. 106, no. 33, pp. 13844-13849, 2009.

[34] M. Pascual, J. A. Ahumada, L. F. Chaves, X. Rodo, and M. Bouma, "Malaria resurgence in the east african highlands: temperature trends revisited," Proceedings of the National Academy of Sciences, vol. 103, no. 15, pp. 5829-5834, 2006.

[35] T. S. Detinova, Age-grouping Methods in Diptera of Medical Importance, with Special Reference to Some Vectors of Malaria, WHO, Geneva, Switzerland, 1962.

[36] G. Macdonald, "The epidemiology and control of malaria," The Epidemiology and Control of Malaria, 1957.

[37] E. A. Mordecai, K. P. Paaijmans, L. R. Johnson et al., "Optimal temperature for malaria transmission is dramatically lower than previously predicted," Ecology Letters, vol. 16, no. 1, pp. 22-30, 2013.

[38] D. Parham, P. E. Pople, C. Christiansen-Jucht, S. Lindsay, W. Hinsley, and E. Michael, "Modeling the role of environmental variables on the population dynamics of the malaria vector anopheles gambiae sensu stricto," Malaria Journal, vol. 11, no. 1, p. 271, 2012.

[39] Microsoft Research, Fetchclimate, http://fetchclimate2. cloudapp.net, 2014.

[40] C. N. Paiva, J. W. de Oliveira Lima, S. S. Camelo, C. de França Lima, and L. P. de Góes Cavalcanti, "Survival of larvivorous fish used for biological control ofAedes aegypti(Diptera: Culicidae) combined with different larvicides," Tropical Medicine \& International Health, vol. 19, no. 9, pp. 1082-1086, 2014.

[41] M. Shahi, E. Kamrani, M. Salehi, R. Habibi, and A. A. Hanafi-Bojd, "Native larvivorous fish in an endemic malarious area of southern Iran, a biological alternative factor for chemical larvicides in malaria control program," Iranian Journal of Public Health, vol. 44, no. 11, p. 1544, 2015.

[42] D. P. Walshe, P. Garner, A. A. Adeel, G. H. Pyke, and T. R. Burkot, "Larvivorous fish for preventing malaria transmission," Cochrane Database of Systematic Reviews, no. 12, 2017.

[43] W. E. Walton, "Larvivorous fish including gambusia," Journal of the American Mosquito Control Association, vol. 23, no. sp2, pp. 184-220, 2007.

[44] World Health Organization, "Use of fish for mosquito control regional office for the Eastern Mediterranean," Technical report, WHO, Geneva, Switzerland, 2003.

[45] E. M. d. M. Santos, L. N. Regis, M. H. N. L. Silva-Filha et al., "The effectiveness of a combined bacterial larvicide for mosquito control in an endemic urban area in Brazil," Biological Control, vol. 121, pp. 190-198, 2018.

[46] Y. A. Derua, E. J. Kweka, W. N. Kisinza, A. K. Githeko, and F. W. Mosha, "Bacterial larvicides used for malaria vector control in sub-saharan africa: review of their effectiveness and 
operational feasibility," Parasites \& Vectors, vol. 12, no. 1, p. $426,2019$.

[47] L. Regis, S. B. d. Silva, and M. A. V. Melo-Santos, "The use of bacterial larvicides in mosquito and black fly control programmes in Brazil," Memórias Do Instituto Oswaldo Cruz, vol. 95, no. 1, pp. 207-210, 2000.

[48] T. Setha, N. Chantha, S. Benjamin, and D. Socheat, "Bacterial larvicide, bacillus thuringiensis israelensis strain am 65-52 water dispersible granule formulation impacts both dengue vector, aedes aegypti (1.) population density and disease transmission in Cambodia," PLoS Neglected Tropical Diseases, vol. 10, no. 9, 2016.

[49] F. B. Agusto, S. Y. Del Valle, K. W. Blayneh et al., "The impact of bed-net use on malaria prevalence," Journal of Theoretical Biology, vol. 320, pp. 58-65, 2013.

[50] A. Abdelrazec, S. Lenhart, and H. Zhu, "Dynamics and optimal control of a west nile virus model with seasonality," The Canadian Applied Mathematics Quarterly, vol. 23, no. 4, pp. 12-33, 2015.

[51] F. B. Agusto, N. Marcus, and K. O. Okosun, "Application of optimal control to the epidemiology of malaria," Electronic Journal of Differential Equations, vol. 2012, no. 81, pp. 1-22, 2012.

[52] C. J. Silva and D. F. M. Torres, "An optimal control approach to malaria prevention via insecticide-treated nets," Conference Papers in Science, vol. 2013, Article ID 658468, 2013.

[53] F. Agusto and S. Lenhart, "Optimal control of the spread of malaria superinfectivity," Journal of Biological Systems, vol. 21, no. 4, Article ID 1340002, 2013.

[54] F. B. Agusto, "Optimal isolation control strategies and costeffectiveness analysis of a two-strain avian influenza model," BioSystems, vol. 113, no. 3, pp. 155-164, 2013.

[55] S. Lenhart and J. T. Workman, Optimal Control Applied to Biological Models, CRC Press, Boca Raton, FL, USA, 2007.

[56] MATLAB 2016A, The Mathworks, Inc, Natick, MA, USA, 2016, http://www.mathworks.com.

[57] N. Chitnis, J. M. Cushing, and J. M. Hyman, "Bifurcation analysis of a mathematical model for malaria transmission," SIAM Journal on Applied Mathematics, vol. 67, no. 1, pp. 24-45, 2006.

[58] Y. Lou and X.-Q. Zhao, "A climate-based malaria transmission model with structured vector population," SIAM Journal on Applied Mathematics, vol. 70, no. 6, pp. 2023-2044, 2010.

[59] K. G. Barnes, G. D. Weedall, M. Ndula et al., "Genomic footprints of selective sweeps from metabolic resistance to pyrethroids in african malaria vectors are driven by scale up of insecticide-based vector control," PLoS Genetics, vol. 13, no. 2, Article ID e1006539, 2017.

[60] C. Wondji, "Scaled-up malaria control efforts breed insecticide resistance in mosquitoes: resistance has swept through mosquito populations in southern africa, threatening malaria prevention," ScienceDaily.

[61] World Health Organization, World Malaria Report 2017, WHO, Geneva, Switzerland, 2017, http://apps.who.int/iris/ bitstream/handle/10665/259492/9789241565523-eng.pdf? sequence $=1$.

[62] World Health Organization, Global Report on Insecticide Resistance in Malaria Vectors: 2010-2016, WHO, Geneva, Switzerland, 2018, http://apps.who.int/iris/bitstream/handle/ 10665/272533/9789241514057-eng.pdf?ua=1.

[63] H. Alout, I. Djègbè, F. Chandre et al., "Insecticide exposure impacts vector-parasite interactions in insecticide-resistant malaria vectors," Proceedings of the Royal Society B: Biological Sciences, vol. 281, no. 1786, Article ID 20140389, 2014.
[64] H. Alout, P. Labbé, F. Chandre, and A. Cohuet, "Malaria vector control still matters despite insecticide resistance," Trends in Parasitology, vol. 33, no. 8, pp. 610-618, 2017.

[65] A. Saddler, P.-C. Burda, and J. C. Koella, "Resisting infection by plasmodium berghei increases the sensitivity of the malaria vector anopheles gambiae to ddt," Malaria Journal, vol. 14, no. 134, 2015.

[66] H. Alout, B. Yameogo, L. S. Djogbenou et al., "Interplay between plasmodium infection and resistance to insecticides in vector mosquitoes," Journal of Infectious Diseases, vol. 210, no. 9, pp. 1464-1470, 2014.

[67] H. Alout, B. Roche, R. K. Dabire, and A. Cohuet, "Consequences of insecticide resistance on malaria transmission," PLoS Pathogen, vol. 13, no. 9, Article ID e1006499, 2017.

[68] World Health Organization, Insecticide Resistance, WHO, Geneva, Switzerland, 2020, https://www.who.int/malaria/ areas/vector_control/insecticide_resistance/en/.

[69] K. Baltzell, K. Harvard, M. Hanley, R. Gosling, and I. Chen, "What is community engagement and how can it drive malaria elimination? case studies and stakeholder interviews," Malaria Journal, vol. 18, no. 1, p. 245, 2019.

[70] F. Bartumeus, G. B. Costa, R. Eritja et al., "Sustainable innovation in vector control requires strong partnerships with communities," PLoS Neglected Tropical Diseases, vol. 13, no. 4, 2019.

[71] C. E. Schairer, R. Taitingfong, O. S. Akbari, and C. S. Bloss, "A typology of community and stakeholder engagement based on documented examples in the field of novel vector control," PLoS Neglected Tropical Diseases, vol. 13, no. 11, 2019.

[72] J. Yu, "Modeling mosquito population suppression based on delay differential equations," SIAM Journal on Applied Mathematics, vol. 78, no. 6, pp. 3168-3187, 2018.

[73] J. Yu and J. Li, "Global asymptotic stability in an interactive wild and sterile mosquito model," Journal of Differential Equations, vol. 269, no. 7, pp. 6193-6215, 2020.

[74] M. Barik, I. Bhattacharjee, A. Ghosh, and G. Chandra, "Larvivorous potentiality of puntius tetrazona and hyphessobrycon rosaceus against culex vishnui subgroup in laboratory and field based bioassay," BMC Research Notes, vol. 11, no. 1, p. 804, 2018.

[75] G. Benelli, C. Jeffries, and T. Walker, "Biological control of mosquito vectors: past, present, and future," Insects, vol. 7, no. 4, p. 52, 2016.

[76] G. Chandra, I. Bhattacharjee, S. N. Chatterjee, and A. Ghosh, "Mosquito control by larvivorous fish," Indian Journal of Medical Research, vol. 127, no. 1, p. 13, 2008.

[77] G. Chandra, S. K. Mandal, A. K. Ghosh, D. Das, S. S. Banerjee, and S. Chakraborty, "Biocontrol of larval mosquitoes by acilius sulcatus (coleoptera: Dytiscidae)," BMC Infectious Diseases, vol. 8, no. 1, p. 138, 2008.

[78] M. Das, M. K. Rao, and A. Kulsreshtha, "Native larvivorous fish diversity as a biological control agent against mosquito larvae in an endemic malarious region of ranchi district in Jharkhand, India," Journal of Vector Borne Diseases, vol. 55, no. 1, p. 34, 2018.

[79] K. Walker and M. Lynch, "Contributions of anopheles larval control to malaria suppression in tropical africa: review of achievements and potential," Medical and Veterinary Entomology, vol. 21, no. 1, pp. 2-21, 2007.

[80] Environmental Protection Agency, "Fact sheet: Bacillus sphaericus 2362, serotype h5a5b, strain am614 (pc code 119804)," EPA, Washington, DC, USA, 2014, https://www3.epa.gov/pesticides/chem_search/reg actions/registration/fs_PC-119803_06-May-14.pdf. 
[81] L. S. Pontryagin, Mathematical Theory of Optimal Processes, CRC Press, Boca Raton, FL, USA, 1987.

[82] W. H. Fleming and R. W. Rishel, Deterministic and Stochastic Optimal Control, Springer Science \& Business Media, Berlin, Germany, 2012. 